\title{
2016 ANNUAL REPORT ARGONNE LEADERSHIP COMPUTING FACILITY
}

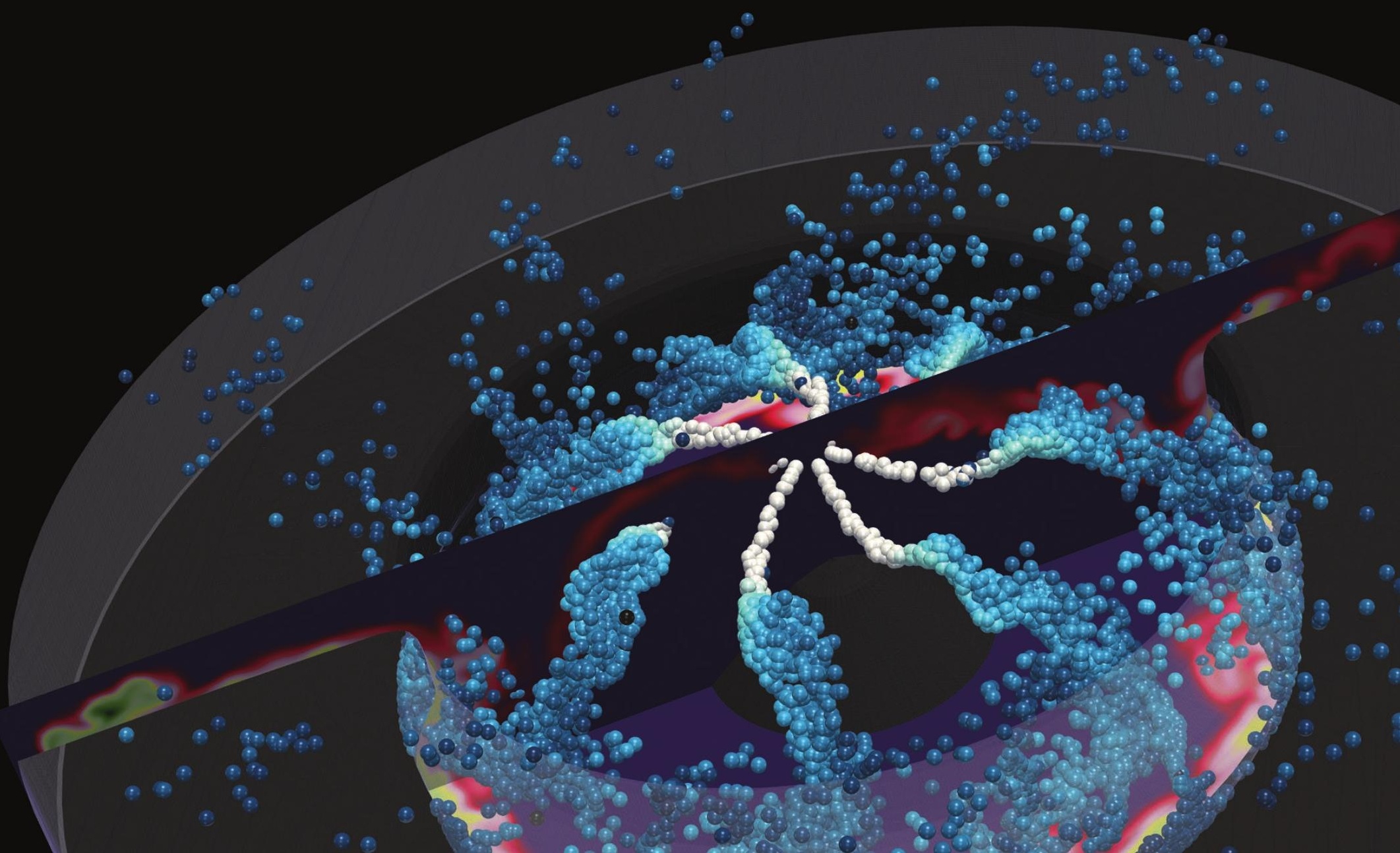


On the cover: This simulation captures fuel distribution inside the combustion chamber of a GM $1.9 \mathrm{~L}$ diesel engine being operated in an advanced lowtemperature combustion mode. Highfidelity 3D simulations provide unique insights into the fuel-air mixing process, which has enabled Argonne engineers to develop this combustion mode. The simulation was carried out on Mira, the ALCF's IBM Blue Gene/Q supercomputer, using the computational fluid dynamics software CONVERGE.

Image credit: Joseph A. Insley, Janardhan Kodavasal, and Sibendu Som, Argonne

National Laboratory 
ARGONNE LEADERSHIP COMPUTING FACILITY ANNUAL REPORT 2016 


\title{
TABLE OF CONTENTS
}

\author{
YEAR IN REVIEW \\ 5 Director's Message \\ 6 About ALCF \\ 8 ALCF Leadership \\ 10 A Decade of Growth and Impact
}

\section{SHAPING THE FUTURE}

16 ALCF's Next-Generation Supercomputers

18 Theta: Paving the Way to Aurora

19 Theta Early Science Projects

20 Early Science Teams Prepare Theta for Science on Day One

22 Beyond Aurora: The Push Toward Exascale

24 Evaluating Future Computing Technologies

26 ALCF's New Data Science Program Targets Big Data

28 Driving Innovation and Competitiveness for U.S. Industry

\section{COMMUNITY \& OUTREACH}

32 Propelling the HPC Community

34 Engaging Current and Future Users

\section{SCIENCE}

40 Accessing ALCF Resources

42 Computing 3D Structures of RNA from Small-Angle X-Ray Scattering Data and Secondary Structures • Yuba Bhandari and Yun-Xing Wang

43 Anomalous Density Properties and Ion Solvation in Liquid Water: A Path-Integral Ab Initio Study • Robert A. DiStasio

44 Towards Breakthroughs in Protein Structure Calculation and Design • David Baker 
45 Frontiers in Planetary and Stellar Magnetism Through High-Performance Computing • Jonathan Aurnou

46 Novel Reduced-Order Models of Turbulent Jet Noise from High-Fidelity Simulation • Joseph Nichols

47 Predictive Modeling of Functional Nanoporous Materials • J. Ilja Siepmann

48 QMC Simulations Database for Predictive Modeling and Theory • David Ceperley

49 Reactive MD Simulations of Electrochemical Oxide Interfaces at Mesoscale - Subramanian Sankaranarayanan

50 Particle Acceleration in Shocks: From Astrophysics to Laboratory In Silico • Frederico Fiuza

51 Predicting the Terascale On-Demand with High-Performance Computing • Radja Boughezal

\section{EXPERTISE \& RESOURCES}
54 ALCF Expertise
56 Staff News
58 Staff Spotlights
60 ALCF Software Resources
62 ALCF Computing Resources

\section{APPENDICES}

64 Publications

82 Projects 


\section{THE YEAR IN REVIEW}

The Argonne Leadership

Computing Facility (ALCF)

helps researchers solve some

of the world's largest and most

complex problems, while

also advancing the nation's

efforts to develop future

exascale computing systems.

This report presents some

of the ALCF's notable

achievements in key strategic

areas over the past year. 


\section{DIRECTOR'S MESSAGE}

The year 2016 marked the ALCF's 10th year in existence - a major milestone for a premier high-performance computing facility whose computing resources have pushed boundaries in science and engineering in ways and at speeds once believed impossible.

In the span of a single decade, we have deployed a succession of supercomputers that have gone from the terascale to petascale. We've helped modernize and optimize key community codes used across a wide range of disciplines. We've forged partnerships to help U.S. companies stay competitive. And we have 10 years of experience serving a scientific community with an insatiable appetite for what's next and what's possible.

It's a good a time to reflect on how far we've come as an organization, and how much we've accomplished.

Each new system the ALCF rolls out represents the state of the art in computing power and capabilities. This continued evolution is allowing researchers to tackle increasingly complex problems, gain insights more quickly, and perform more precise simulations of everything from combustion engines to the human brain.

The recent arrival of our newest system, Theta, has given us a glimpse of the future of leadership computing. Project teams from the Theta Early Science Program, the first researchers to gain access to the new system, are already reporting notable performance gains. Meanwhile, our primary production supercomputer, Mira, continues to serve as a reliable and powerful tool for many scientific achievements, including the development of a new coating material to reduce friction and the design of novel peptides to advance tailored drug design.

This year we also kicked off the ALCF Data Science Program, a pioneering initiative aimed at preparing data-centric applications for leadership computing resources. Forward-looking efforts like the ADSP, and others, are helping our nation maintain its leadership role in advancing scientific discovery through high-performance computing.

As we look ahead to another exciting year in 2017, I would like to thank our staff, our users, and our collaborators for making the ALCF what it is today. And while we have seen breakthrough after breakthrough in the past decade, the next decade will be an even more exciting time for science and technology.

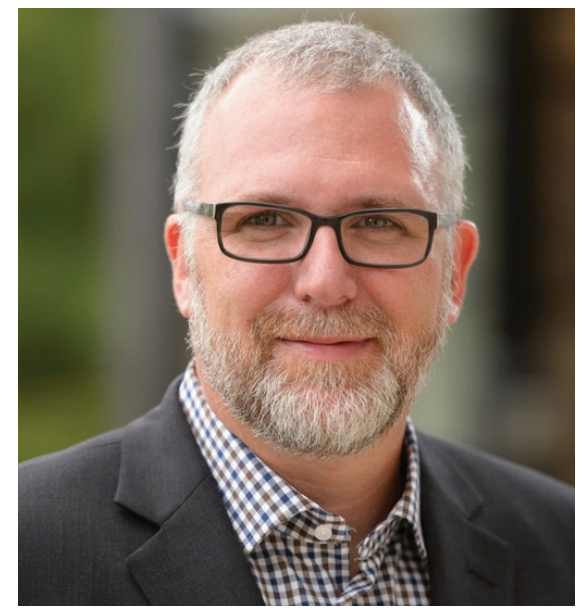

\section{Michael E. Papka}

Division Director, ALCF;

Deputy Associate Laboratory Director, Computing, Environment, and Life Sciences 


\section{ABOUT ALCF}

\section{STATS}

\section{The Argonne Leadership \\ Computing Facility provides \\ supercomputing capabilities \\ to the scientific and engineering \\ community to advance \\ fundamental discovery and understanding in a broad range of disciplines.}

Supported by the U.S. Department of Energy's (DOE) Office of Science, Advanced Scientific Computing Research (ASCR) program, the ALCF is one of two DOE Leadership Computing Facilities in the nation dedicated to open science.

Available to researchers from universities, industry, and government agencies, the ALCF is a DOE Office of Science User Facility that helps accelerate the pace of discovery and innovation by providing supercomputing resources that are 10 to 100 times more powerful than systems typically used for scientific research.

Through substantial awards of supercomputing time and user support services, the ALCF enables large-scale modeling and simulation research aimed at solving some of the world's largest and most complex problems in science and engineering.

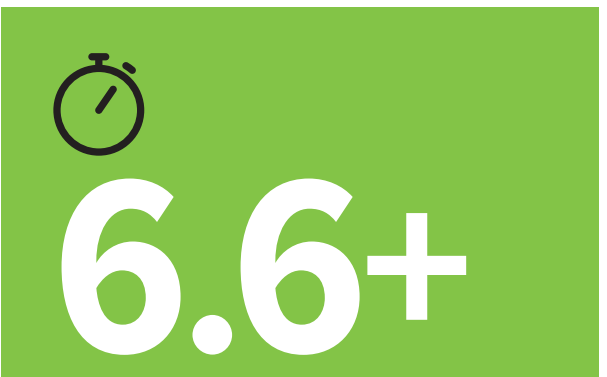

\section{BILLION CORE-HOURS OF TOTAL COMPUTE TIME}

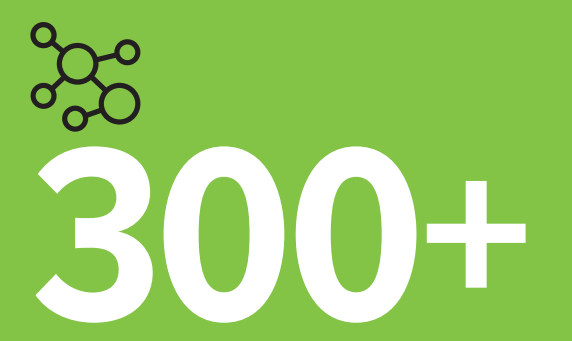

ACTIVE PROJECTS*

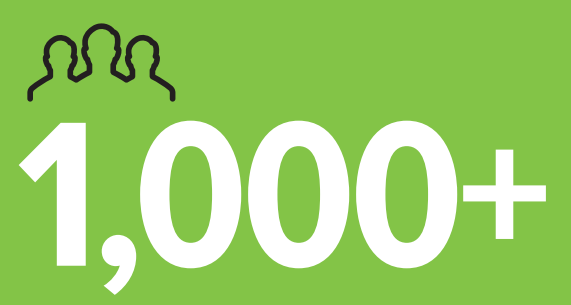

FACILITY USERS*

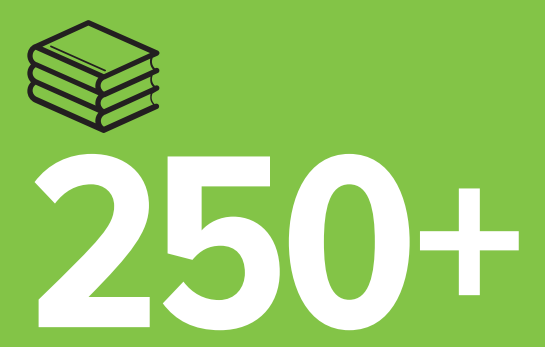

PUBLICATIONS 


\section{ALCF USERS BY STATE}

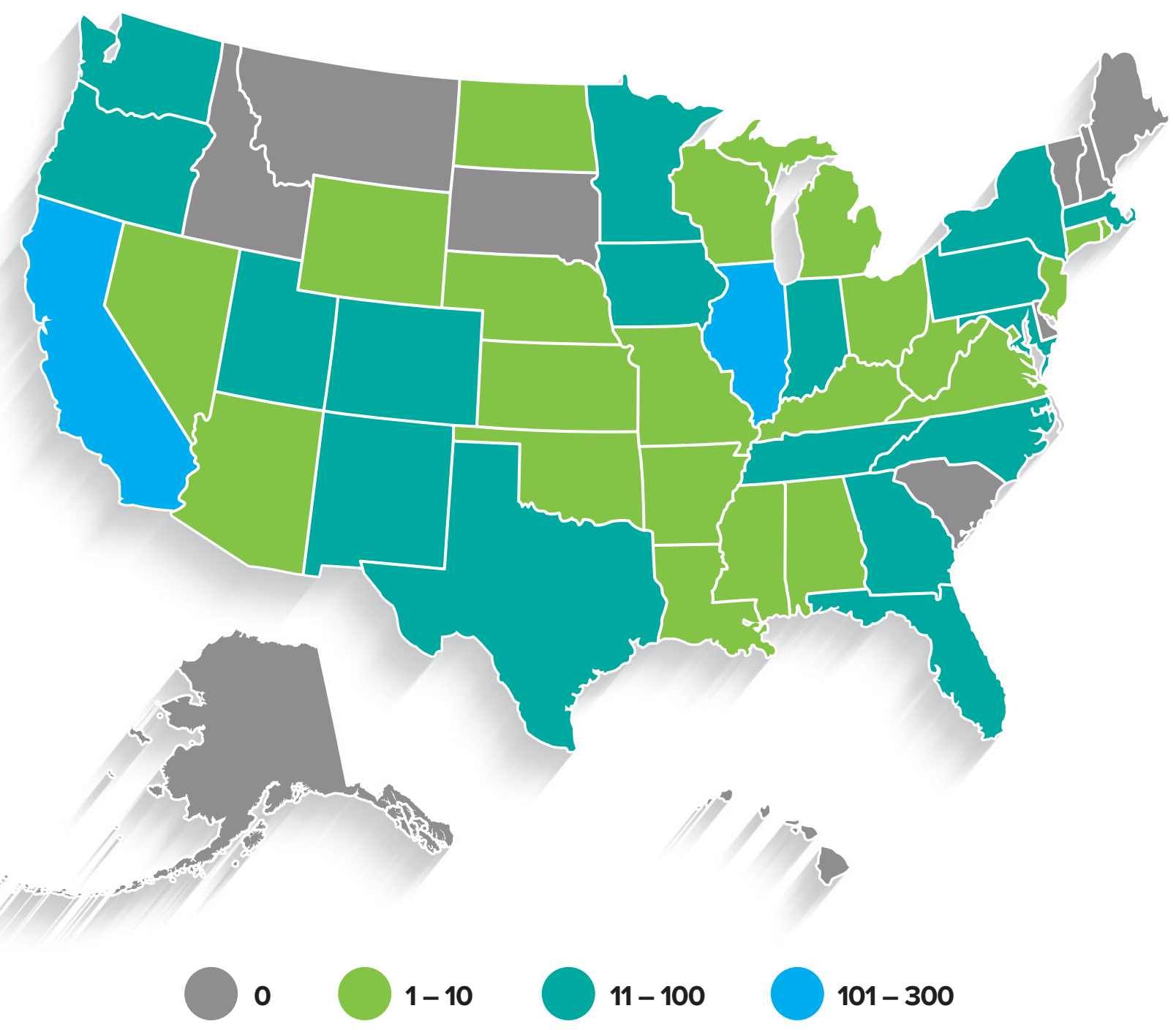

\section{ALCF USERS BY AFFILIATION}

ACADEMIA

GOVERNMENT

INDUSTRY

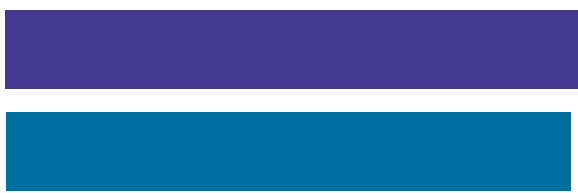

81

581

\section{7}




\section{ALCF LEADERSHIP}

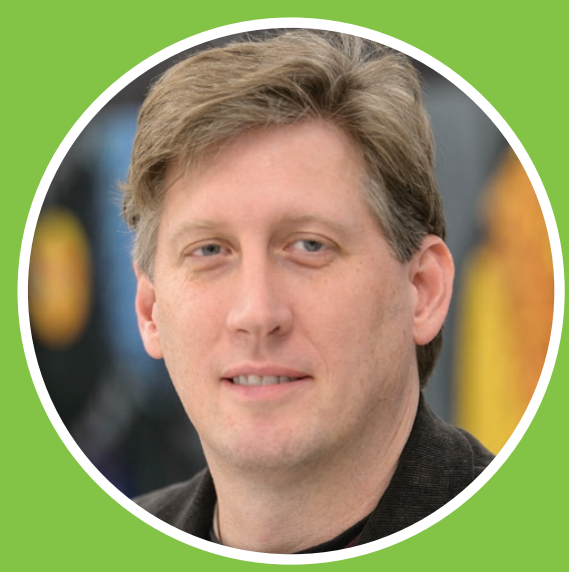

OPERATIONS

\section{Mark Fahey}

DIRECTOR OF OPERATIONS
Advanced Integration, HPC Infrastructure, HPC Storage, HPC Systems \& Network Administration

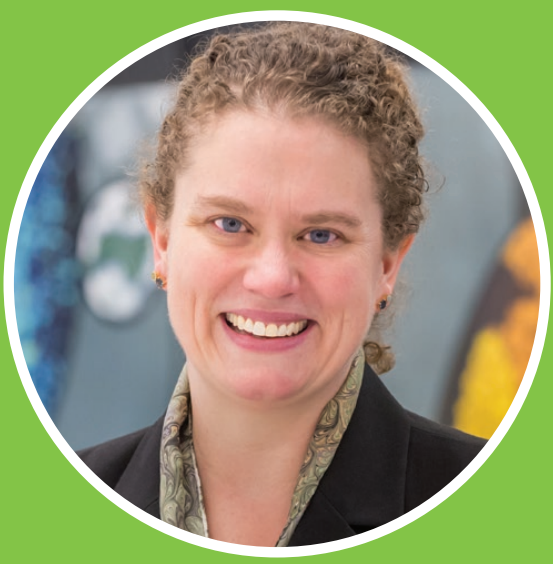

SCIENCE

\section{Katherine Riley}

DIRECTOR OF SCIENCE
Computational Science, Data Sciences, Performance Engineering, Visualization \& Data Analysis

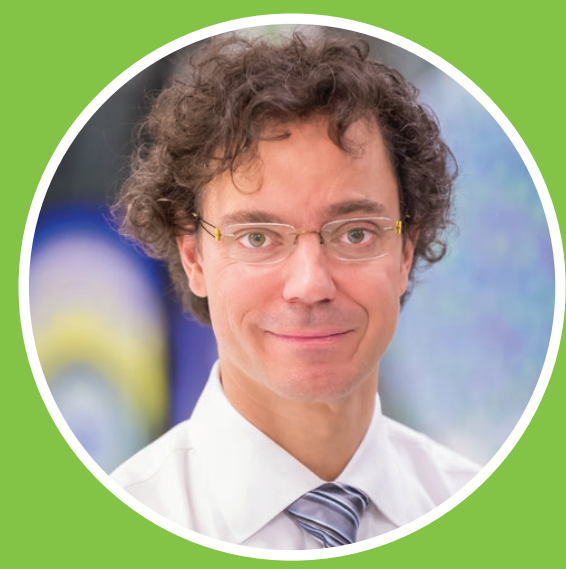

USER EXPERIENCE Richard Coffey DIRECTOR OF USER EXPERIENCE
Business Intelligence, Communications, Industrial Outreach, Technical Support 


\section{ALCF teams play a critical role in supporting the facility's supercomputing environments, the user community, and their efforts to accelerate scientific discoveries.}

\section{OPERATIONS}

Mira had another excellent year in 2016, exceeding all key metrics for availability and utilization. Our new system, Theta, was delivered and installed this summer. Thanks to the dedication and expertise of our acceptance team, who worked closely with staff from Intel and Cray to debug and address technical issues, Theta was accepted six months ahead of schedule. We also successfully ported our Cobalt scheduler to Theta so it was ready to run jobs as soon as the system was released to Early Science Program users.

We made several upgrades to our storage capabilities in 2016. To improve our archival capacity, we installed a third eight-frame tape library with approximately 24 petabytes using LTO6 media. We deployed the GPFS/HPSS Interface (GHI) file migration tool on one of our scratch filesystems, and will soon deploy it on the other filesystem. Members of our team also developed and launched Bloodhound, a systemmonitoring tool that performs wellness checks on our storage systems and provides continuous alerts on critical hardware and software changes. Additionally, we launched sbank, a new allocation accounting system that provides users with more flexibility in querying project usage data.

In the coming year, we will bring Theta into full production mode, while continuing to prepare for the arrival of Aurora. We also expect to deploy our burst buffer-like storage cache, and new data transfer nodes and data movers for the archives.

- Mark Fahey

\section{SCIENCE}

While Theta's arrival generated some new excitement in 2016, it was Mira that continued enabling our user community to pursue science and engineering breakthroughs. In 2016 , ALCF project teams produced more than 250 research publications, including papers in high-impact journals such as Science, Nature, and Physical Review Letters. Theta did get some use as well, as researchers from our Early Science Program achieved notable performance gains and scaling results while preparing for their science campaigns. Next year, we'll be kicking off the Early Science Program for Aurora, and continuing our close collaboration with Intel and Cray to prepare applications and our user community for the facility's nextgeneration supercomputers.

In our rapidly evolving community, it's critical that we operate with an eye on the future. This year, we were involved in some key efforts aimed at helping the nation move closer to realizing a future exascale system, including DOE's Exascale Requirements Reviews and the Exascale Computing Project (ECP). For the ECP effort several members of our team will continue to be deeply engaged in projects to develop applications and software for exascale machines. In addition, we launched the ALCF Data Science Program, as well as a new data science team, to explore and improve the computational tools and techniques needed to enable future data-driven discoveries on leadership computing resources.

- Katherine Riley

\section{USER EXPERIENCE}

The User Experience team is responsible for making sure ALCF users have a positive and productive experience with our facility, our services, and our supercomputers. Our goal is to help to reduce friction, remove roadblocks, and support the needs and goals of the scientists and engineers who use our leadership computing resources.

Stewarding the large-scale projects that are awarded computing time at the ALCF requires a significant amount of communication and assistance to the principal investigators and their teams. By using best practices and continuous improvement techniques, we ensured project teams were on-boarded quickly and efficiently so they could begin their science campaigns as soon as their allocations began. We also helped bring our users' stories to life by communicating the impact of their work to the scientific and HPC communities, our sponsors, and the general public.

On the outreach side, we connected the ALCF to a variety of new industry users, extended our user training offerings, and hosted the annual meeting of the Intel ${ }^{\circledR}$ Xeon $\mathrm{Phi}^{\text {Tm }}$ User's Group. We've also been making significant strides with business intelligence. Not only have we improved the efficiency of the ALCF's data management and analysis activities by centralizing all of the facility's critical data, we have ensured our internal and external reports are as accurate as possible.

- Richard Coffey 


\section{0-YEAR ANNIVERSARY}

\section{A DECADE OF \\ GROWTH \\ AND
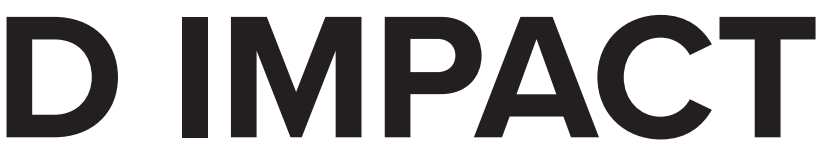

In its first decade of existence, the ALCF has played a significant role in advancing discovery and innovation through high-performance computing.

The ALCF was established at Argonne National Laboratory in 2006 as part of a DOE initiative dedicated to enabling transformational advances in science and engineering by providing the most powerful supercomputers in the world for open scientific research. The facility has grown leaps and bounds in its first decade, developing and deploying leading-edge computing systems with unprecedented capabilities, while fostering an ever-growing user community that is pushing the boundaries of fundamental discovery and understanding in a broad range of scientific disciplines.

TOTAL ALLOCATED HOURS

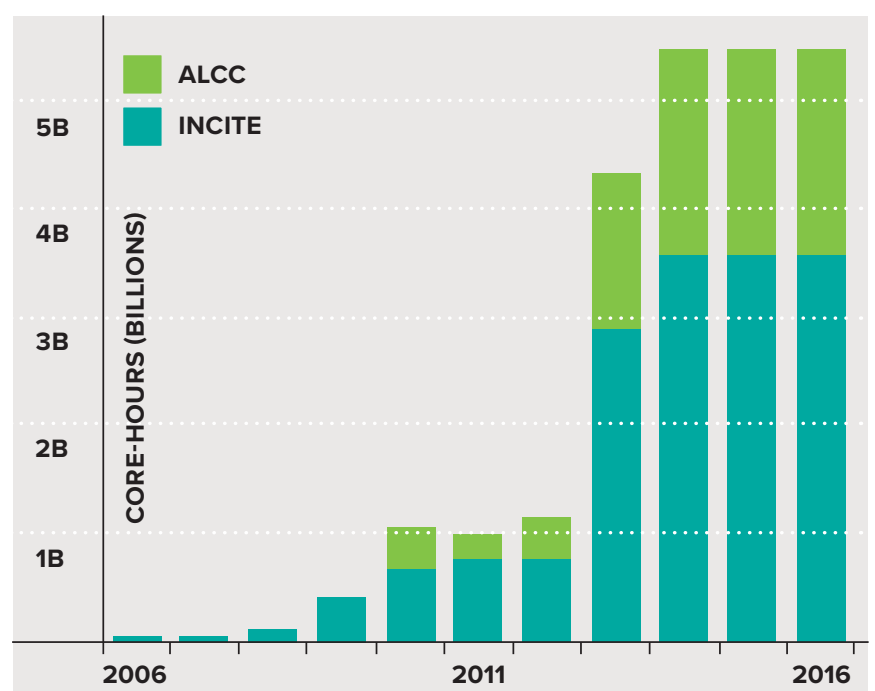

\section{AVERAGE PROJECT SIZE}

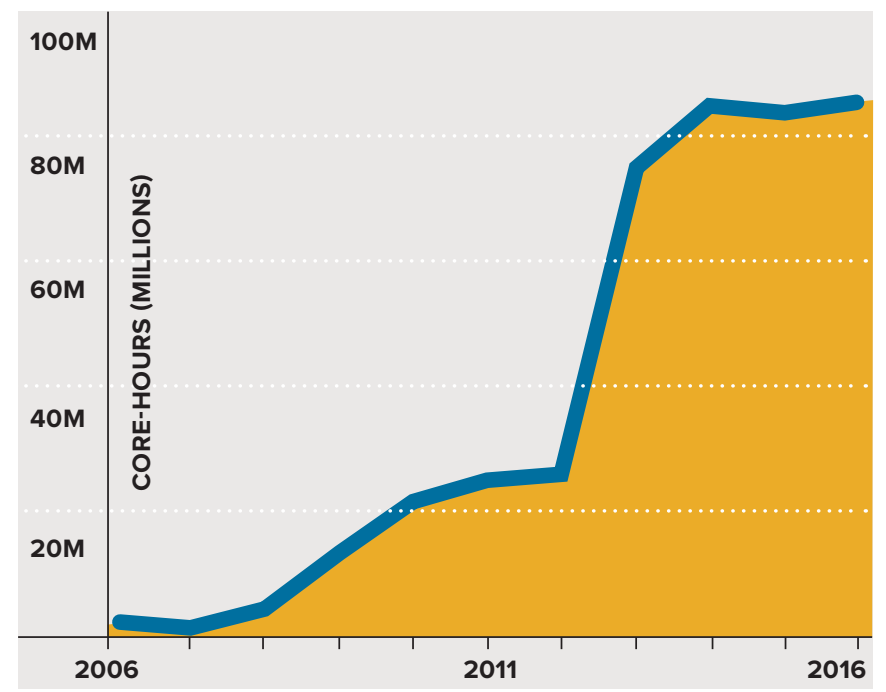

The ALCF's two primary allocation programs - INCITE and ALCC - have grown along with the facility. With each new supercomputer, these programs have awarded increasingly larger allocations of computing time to researchers. 


\section{THE HISTORY OF ALCF SUPERCOMPUTERS}

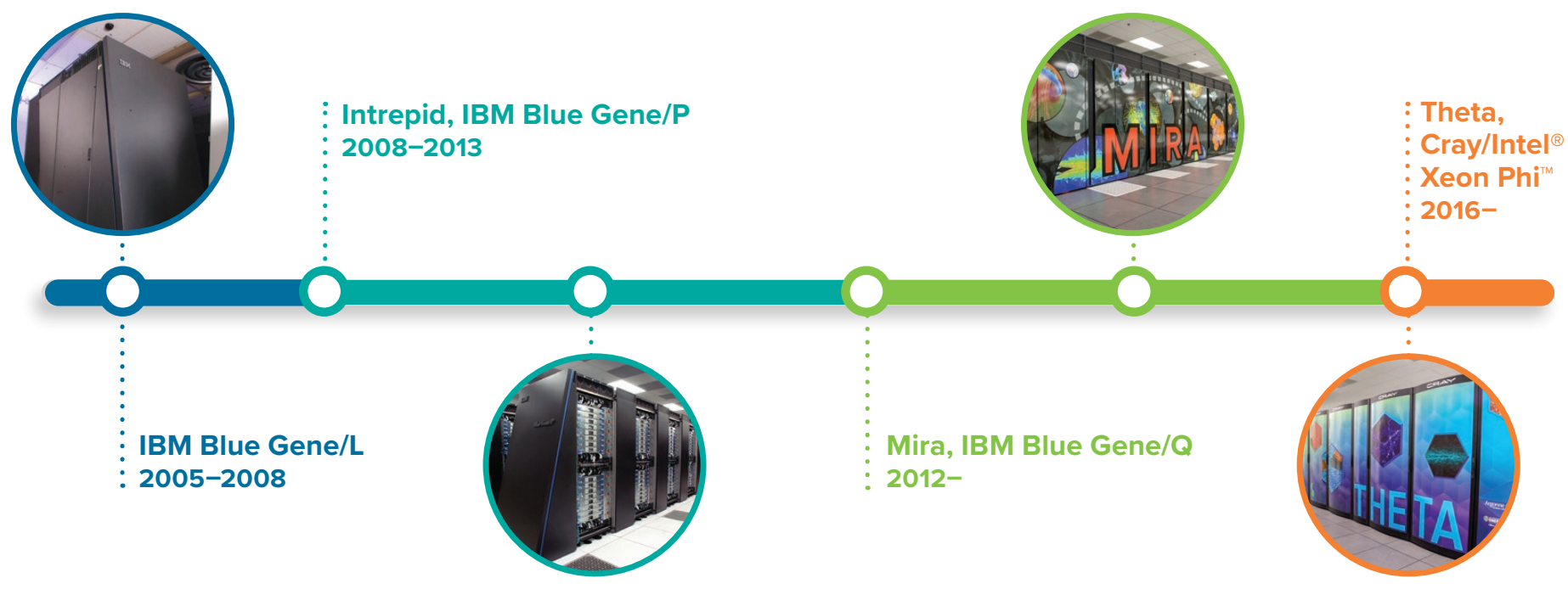

From teraflops to petaflops, ALCF supercomputers have come a long way over the past decade. Each new system brings advanced capabilities that enable researchers to expand their investigations in both scale and scope.

\section{ALCF SYSTEM SPECS}

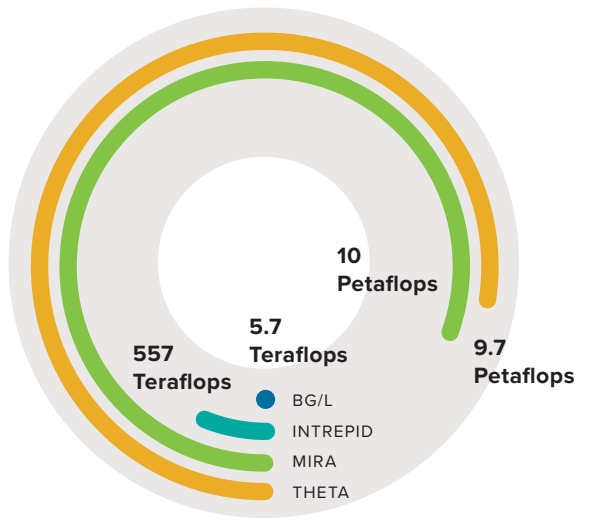

PEAK PERFORMANCE

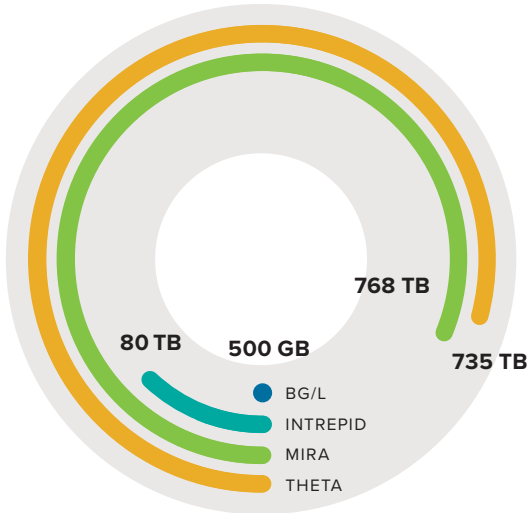

MEMORY

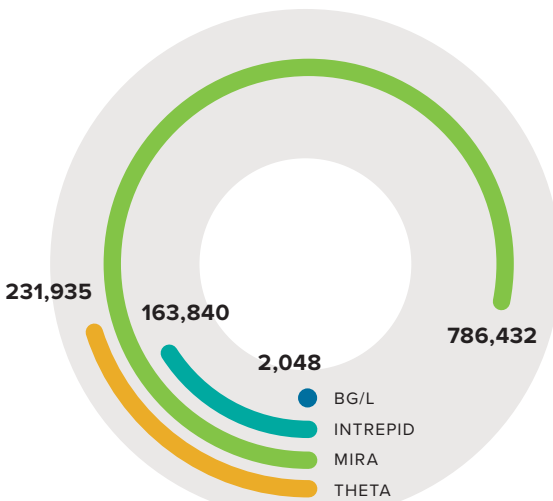

CORES 


\section{0-YEAR ANNIVERSARY}

\section{To acknowledge a decade of breakthrough science, here is a look at some notable accomplishments made possible by the ALCF's computing resources and expertise.}

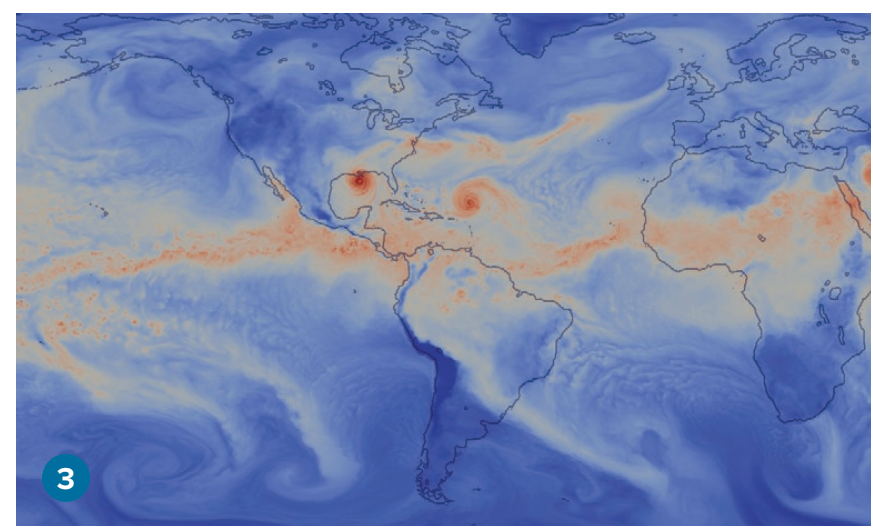

A snapshot from the Community Atmosphere Model. Image credit: Joseph A. Insley, Robert Jacob, and Sheri Mickelson, Argonne National Laboratory; Andy Bauer, Kitware; Mark Taylor, Sandia National Laboratories

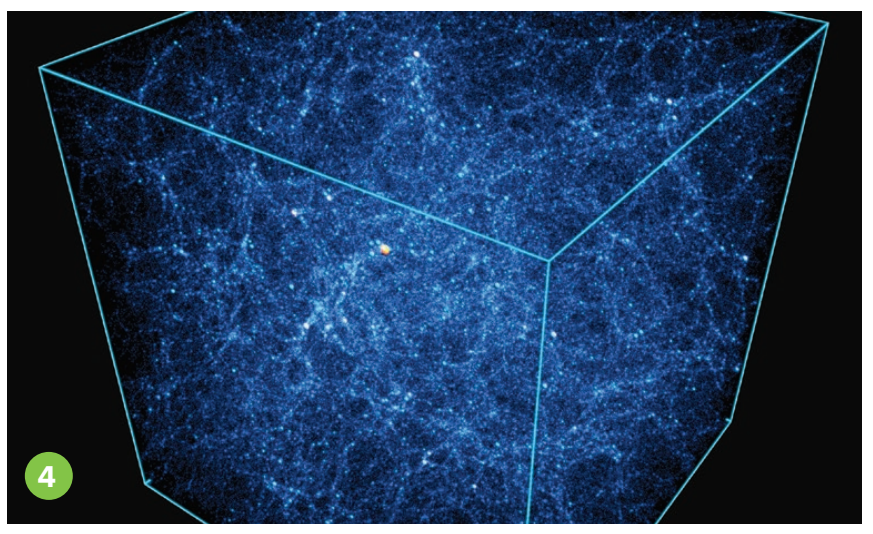

This visualization shows the distribution of matter in the Outer Rim simulation. Image credit: Joseph A. Insley, Silvio Rizzi, and the HACC team, Argonne National Laboratory
ENERGY TECHNOLOGIES

\section{Adding Renewables to the Electric Grid Mihai Anitescu \\ Argonne National Laboratory} Adding renewable energy to the electric grid is tricky, because solar and wind generation fluctuate with weather conditions. A team of researchers used ALCF resources to demonstrate that a complex mathematical approach called stochastic optimization can help grid operators minimize instability and distribute power on demand while minimizing the need for costly backup plants.

\section{MATERIALS SCIENCE}

\section{Developing New Types of Concrete \\ William George}

National Institute of Standards and Technology

Concrete is essential to modern life, but its production releases a significant amount of greenhouse gases. To develop new formulas that reduce emissions, scientists modeled the flow of concrete and found new principles that should help guide the design of more environmentally friendly types of concrete.

EARTH SCIENCE

\section{Enhancing Climate Predictions \\ Warren Washington \\ National Center for Atmospheric Research}

When we want to know how the climate might change in the future - which areas might have more drought, more floods, more heat - we turn to climate models. But predicting the future on a global climate scale is extraordinarily difficult; it's so complex that slight changes to the parameters show different futures. The ALCF has been helping scientists run these enormous climate models and refine the techniques to provide more accurate simulations.

PHYSICS

\section{Illuminating the Early Universe} Salman Habib Argonne National Laboratory The Outer Rim simulation, one of the largest cosmological simulations ever run, modeled the evolution of the universe over billions of years, allowing scientists to "watch" as galaxies develop. This helps researchers understand how cosmic structures are formed and provides a new tool for studying the mysterious force called dark energy.

\section{ENGINEERING}

\section{Improving Airplane Engines Peter Bradley Pratt \& Whitney}

Inside a jet engine, air flowing faster than a hurricane is combined with fuel to generate heat that powers the plane. Designers are turning to supercomputers to model these complex processes to make new engines that are cleaner, quieter, and less expensive. A study at the ALCF was key to helping industry integrate supercomputer simulations into their design process. Improvements based on this work are now currently in use in aircrafts. 


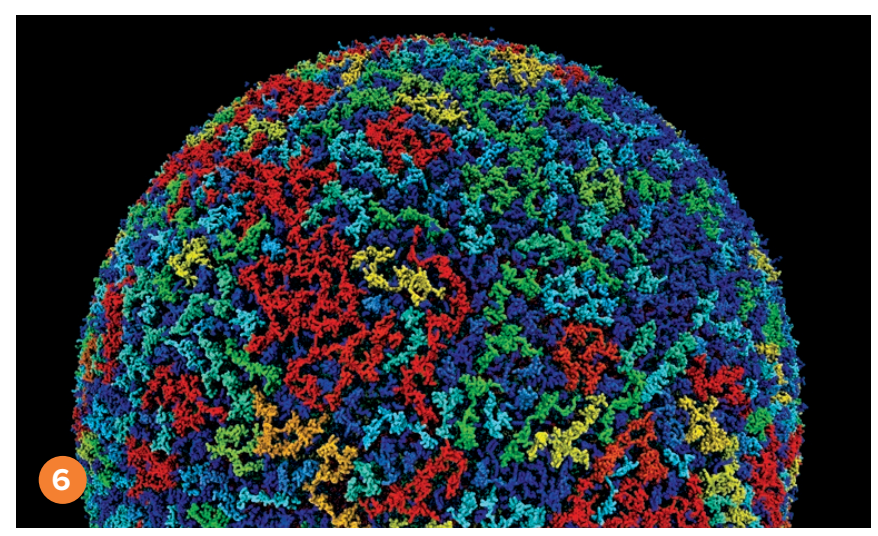

Nanocarbon produced by oxidation of a silicon-carbide nanoparticle. Image credit: Joseph A. Insley, Argonne National Laboratory; Ken-ichi Nomura, University of Southern California

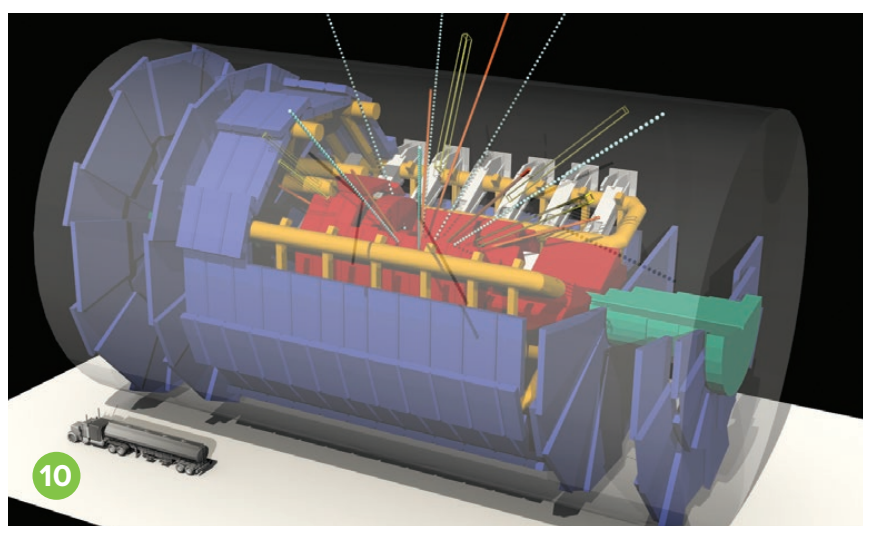

An artist's representation of particle collisions in the ATLAS detector at CERN's Large Hadron Collider. Image credit: Taylor Childers, Joseph A. Insley, and

Thomas LeCompte, Argonne National Laboratory
MATERIALS SCIENCE

\section{Investigating How Materials Crack}

Priya Vashishta and Rajiv Kalia University of Southern California When materials crack and corrode under stress, the process starts at the molecular level. Only supercomputers have the capability to run simulations so detailed that they actually show how molecules propagate a crack over time. These simulations can help researchers design possible solutions, such as self-healing nanomaterials, to build safer, stronger materials for highstress environments.

\section{CHEMISTRY}

\section{Modeling Detonations to Transport Explosives Safely Martin Berzins \\ The University of Utah}

Using ALCF supercomputers, researchers modeled an explosion that left a 30-by-70-foot crater in a Utah highway, capturing the physics that made the truck's cargo explode more violently than it should have. With such simulations, engineers can design safer transport for explosives.
BIOLOGICAL SCIENCES

\section{Puzzling Out Proteins that Work Against Antibiotics}

T. Andrew Binkowski

Argonne National Laboratory

In the race against antibiotic resistance, computing is becoming a valuable tool for identifying new ways to fight disease. Researchers ran simulations on key pathogen proteins, including nine molecules that could work against anthrax. They also explored potential avenues to defeat a gene that confers resistance against even the strongest antibiotics.

\section{BIOLOGICAL SCIENCES}

\section{Understanding the Molecular Basis of Parkinson's Disease Igor Tsigelny}

University of California, San Diego

Researchers ran simulations to understand how several proteins connected with Parkinson's disease interact to cause the degenerative neurological disorder. The findings provide a test bed for identifying possible treatments via computer modeling.

\section{PHYSICS}

\section{Using Supercomputers to Analyze Particle Collisions Thomas LeCompte \\ Argonne National Laboratory}

At the Large Hadron Collider (LHC) in Europe, the most powerful accelerator in the world, physicists run simulations on the data collected from particle collisions in order to understand the fundamental forces that drive the universe. To explore the potential of supercomputers to enable future discoveries at $\mathrm{LHC}$, a research team adapted their code to run on ALCF computing resources, allowing them to analyze more data and simulate more complex events than previously possible. 


\section{SHAPING THE FUTURE}

ALCF researchers lead and participate in several strategic activities that aim to push the boundaries of what's possible in computational science and engineering. 


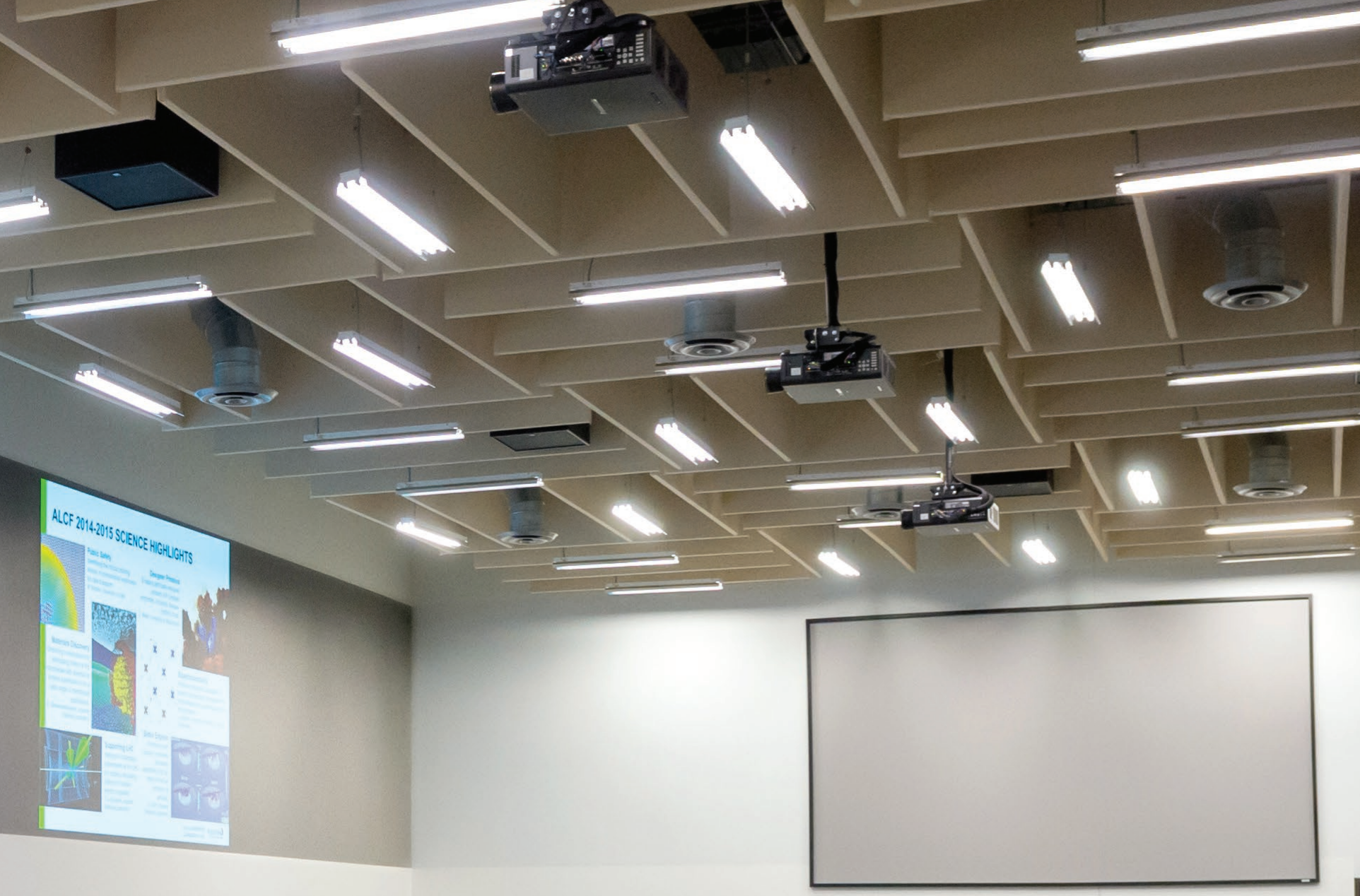

$\neg$

4ix.

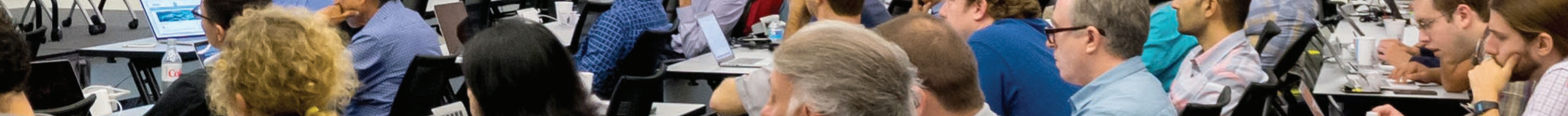

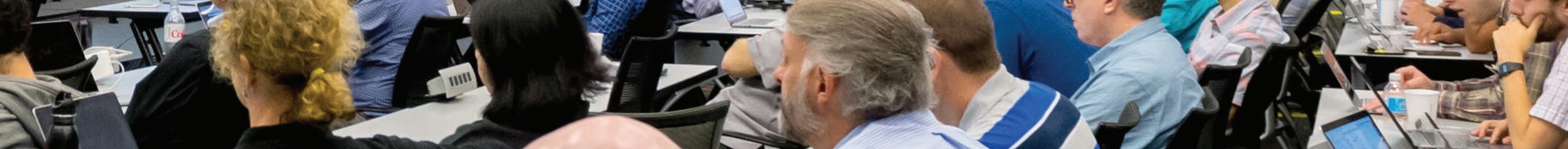

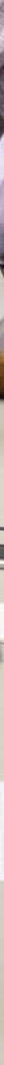




\section{SHAPING THE FUTURE}

\section{ALCF'S NEXT-GENERATION SUPERCOMPUTERS}

\section{The ALCF hits major milestones with the acceptance of Theta and preparations for Aurora.}

In 2016, the ALCF made significant strides toward its next-generation Intel-Cray systems with the successful delivery of Theta and the continued development of Aurora.

Aurora, a 200-petaflops system scheduled for delivery in 2018, will replace Mira as the ALCF's next major production supercomputer. Theta is designed to be a bridge between Mira and Aurora, providing an early Intel-Cray system that will enable new science while helping users transition their applications to the new technology.

Developed in collaboration with industry leaders Intel and Cray, Aurora and Theta are funded under DOE's Collaboration of Oak Ridge,
Argonne, and Lawrence Livermore (CORAL) initiative, a joint procurement activity launched in 2014 to build state-of-the-art supercomputers that are essential for technology advancement and scientific discovery. The new ALCF systems will help ensure continued U.S. leadership in high-end computing for scientific research, while also cementing the nation's position as a global leader in the development of next-generation exascale computing systems.

Among the most notable milestones in 2016 was the successful installation and acceptance of Theta. The system was delivered to Argonne two months ahead of schedule, arriving at the ALCF in late June. Once the system was installed, ALCF staff members, in collaboration with Intel and Cray, worked diligently to integrate and evaluate the machine to prepare it for acceptance testing. The team's work included developing tests and tools to identify, debug, and resolve several technical challenges along the way. ALCF staff members were also instrumental in setting up and running many of the acceptance test codes while working with the vendors to analyze performance and correctness results.
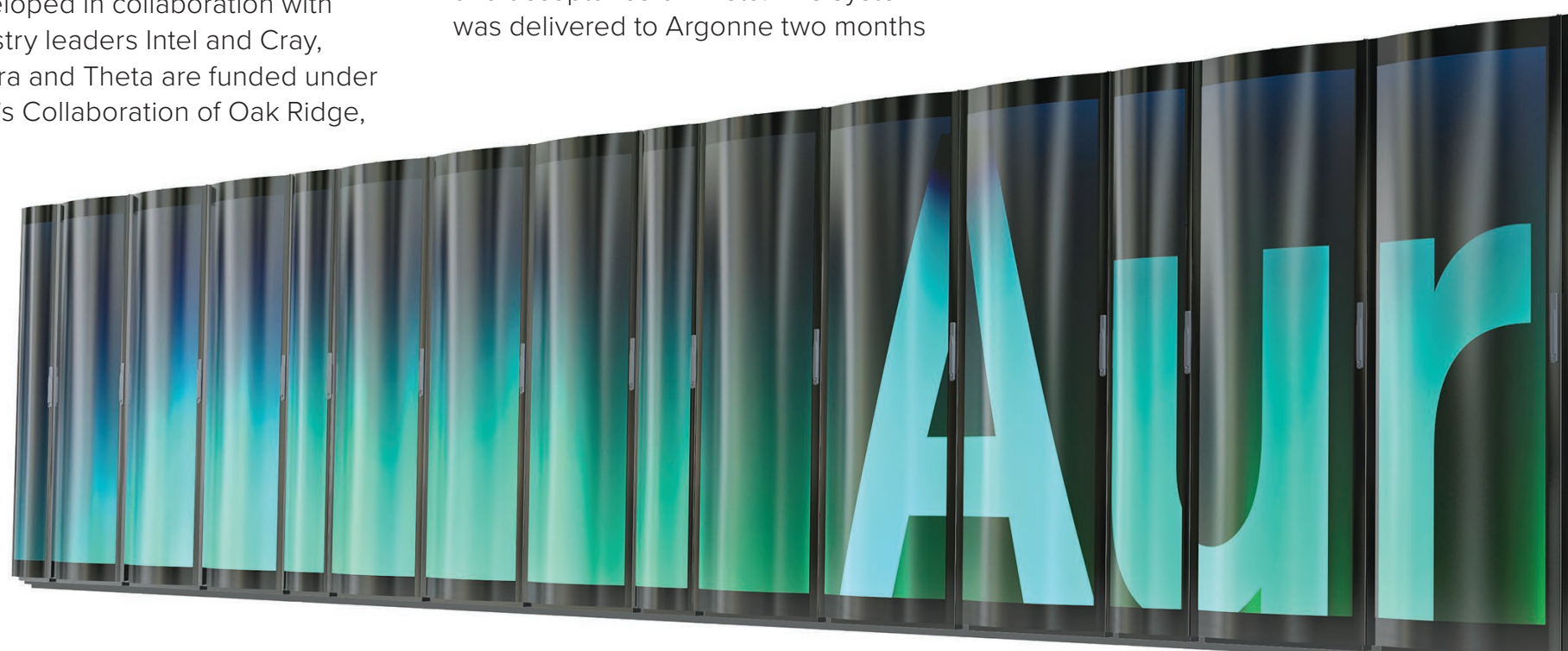
The collaborative efforts resulted in Theta being accepted on September 30, 2016, six months earlier than the initial target date for acceptance. Theta was subsequently made available to research teams in the Theta Early Science Program, and will be opened up to the broader ALCF user community in 2017.

On the Aurora front, the ALCF-IntelCray team made progress with the project's non-recurring engineering (NRE) work, which encompasses activities to enhance the usability and effectiveness of the final system. Much of the work in 2016 focused on facility infrastructure enhancements to prepare for the new supercomputer, including the installation of additional cooling capacity at Argonne's chilled water plant, an upgrade to Argonne's power station to accommodate Aurora's power requirements, and the completion of design plans for Aurora's electrical and cooling systems at Argonne's Theory and Computing Sciences Building, where the system will be installed. In addition, ALCF staff continues to work closely with the vendors to enhance the system's architecture, packaging, and software stack.

In July 2016, the ALCF issued a Call for Proposals for its Aurora Early Science Program, with the selected projects to be announced in early 2017. The program will award computing time to 10 science teams to pursue innovative research as part of pre-production testing on the new system. The projects will prepare key applications for the architecture and scale of Aurora and solidify libraries and infrastructure to pave the way for other applications to run on the system. To develop and optimize their applications for Aurora, project teams will be given access to early hardware, receive training on the underlying Intel and Cray technologies, and work collaboratively with ALCF staff and vendor experts.

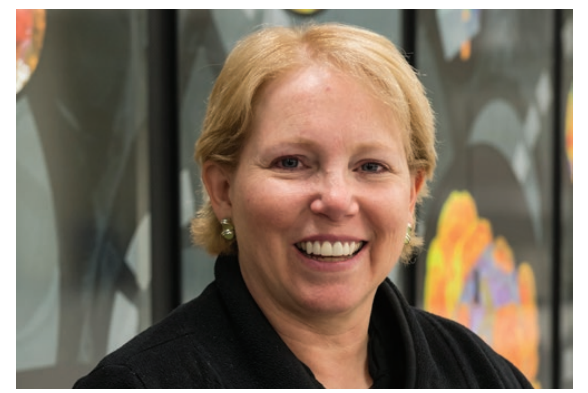

"With the development of Theta and Aurora, we were able to continue evolving the many-core, homogenous architectures that our user community has grown accustomed to. These leading-edge systems will fuel a new era of scientific discoveries at the ALCF." - Susan Coghlan, project director for Theta and Aurora 


\section{SHAPING THE FUTURE}

\section{THETA: PAVING THE WAY TO AURORA}

\section{Theta, the ALCF's new 9.65 petaflops supercomputer, will help bridge the gap between Mira and Aurora.}

Designed in collaboration with Intel and Cray, Theta provides advanced capabilities that will enable breakthrough computational science and engineering research at the ALCF. The early production system will also help ALCF users transition their applications to the new Intel-Cray architecture.

Theta is designed to serve as a stepping stone to the ALCF's next leadership-class supercomputer, Aurora. Like Theta, Aurora will be a massively parallel, many-core system powered by Intel ${ }^{\circledR}$ Xeon Phi ${ }^{\text {TM }}$ processors, running Cray's HPC software stack.

Theta is equipped with 3,624 nodes, each containing a 64-core processor with 16 gigabytes (GB) of high-bandwidth in-package memory (MCDRAM), 192 GB of DDR4 RAM, and a 128 GB SSD. Theta's initial parallel file system is 10 petabytes.

While Theta's peak speed is slightly less than Mira's, the new system has several features that will allow scientific codes to achieve higher performance. These features include: $\square$ High-bandwidth MCDRAM (300-450 GB/s depending on memory and cluster mode), with many applications running entirely in MCDRAM or using it effectively with dynamic RAM (DRAM)

$\square$ Improved single-thread performance

$\square$ Potentially much better vectorization with AVX 512

$\square$ Large total memory per node (208 GB on Theta vs. 16 GB on Mira)

Theta is located in the machine room in Argonne's Theory and Computing Sciences Building.

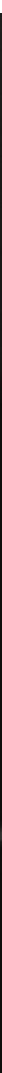




\section{THETA EARLY SCIENCE PROJECTS}

The ALCF's Theta Early Science Program allocates preproduction time on the system to projects pursuing science and code development work.

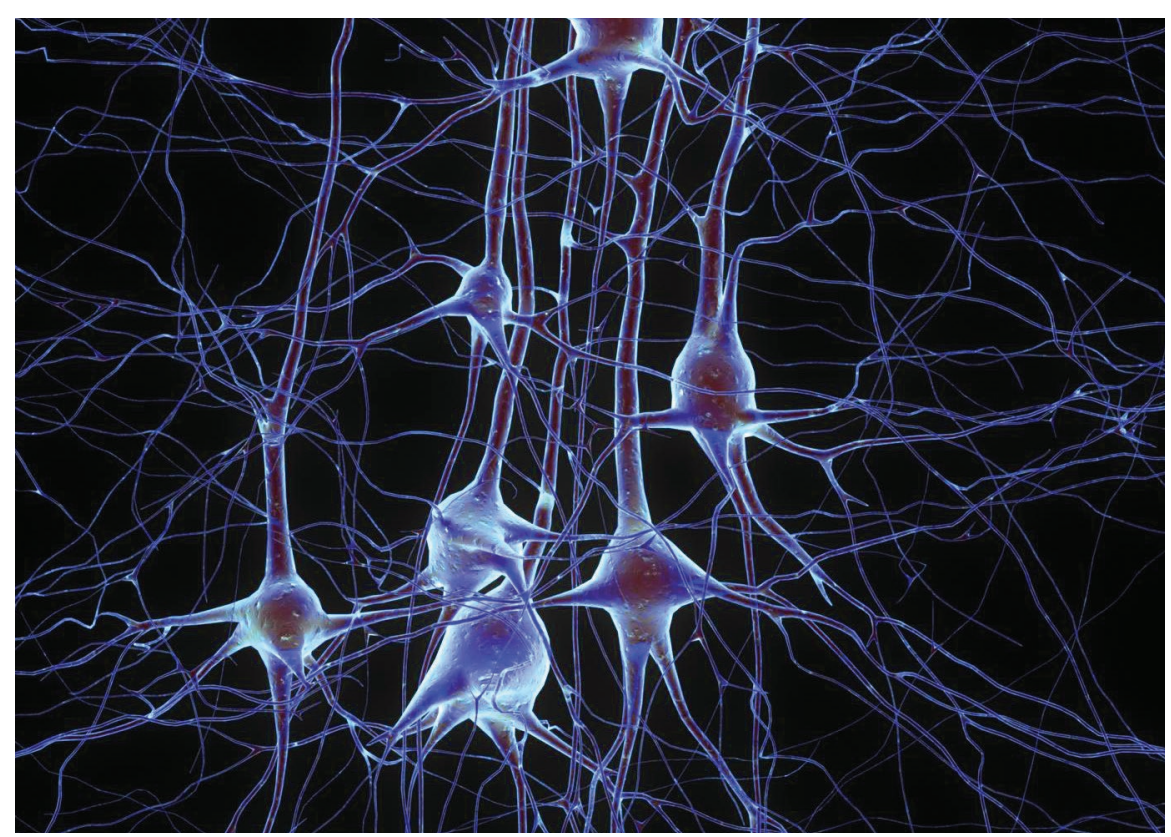

Digital reconstruction of pyramidal cells. Image credit: Blue Brain Project, EPFL

Direct Numerical Simulations of Flame Propagation in Hydrogen-Oxygen Mixtures in Closed Vessels

Alexei Khokhlov, The University of

Chicago

Code: HSCD

\section{Free Energy Landscapes of} Membrane Transport Proteins

Benoît Roux, The University of

Chicago/Argonne National Laboratory

Code: NAMD

\section{Next-Generation Cosmology}

Simulations with HACC:

Challenges from Baryons

Katrin Heitmann, Argonne National

Laboratory

Code: HACC

\section{CODE \\ DEVELOPMENT PROJECTS}

Advanced Electronic Structure Methods for

Heterogeneous Catalysis and Separation of Heavy Metals Mark Gordon, lowa State University Code: GAMESS

Electronic Structure-

Based Discovery of Hybrid Photovoltaic Materials on Next-Generation HPC Platforms

Volker Blum, Duke University Codes: FHI-aims, GAtor

Extreme-Scale Unstructured Adaptive CFD: From Multiphase Flow to Aerodynamic Flow Control Kenneth E. Jansen, University of Colorado Boulder Code: PHASTA

Flow, Mixing, and Combustion of Transient Turbulent Gaseous Jets in Confined Cylindrical Geometries Christos Frouzakis, Swiss Federal Institute of Technology Zurich Code: Nek5000

The Hadronic Contribution to the Anomalous Magnetic Moment of the Muon Paul Mackenzie, Fermilab Codes: MILC, CPS

Quantum Monte Carlo Calculations in Nuclear Theory

Steven Pieper, Argonne National Laboratory Code: GFMC 


\section{SHAPING THE FUTURE}

\section{EARLY SCIENCE TEAMS PREPARE THETA FOR SCIENCE ON DAY ONE}

\section{The ALCF's Theta Early Science Program (ESP) is designed to prepare users for the new supercomputer, ensuring that projects and codes are ready to make good scientific use of the system.}

Researchers participating in the Theta ESP are getting the first crack at using the new supercomputer for science simulations. In the process, they are helping pave the way for other applications to run on the system.

By bringing together computational scientists, code developers, and computing hardware experts, the program creates a collaborative environment for optimizing applications and characterizing the behavior of new hardware and software features.

Long before Theta was installed at Argonne this summer, ESP research teams, in collaboration with ALCF and vendor staff, were working diligently to adapt their applications for the system's new Intel-Cray architecture. Access to advanced Theta simulator software and early hardware allowed the teams to test and debug applications in advance of the system being available. The researchers were also able to use Mira and other ALCF computing resources for early development work that did not require Theta hardware (e.g., new algorithms, new physics modules).

In August, the ALCF hosted a handson workshop that allowed the ESP teams to use the full Theta system to port, benchmark, and optimize their applications. Attendees were able to measure the scaling of their applications on problem sizes relevant to their proposed science runs. They also received instruction on Intel and Cray development environments, system software, the Cobalt job scheduler, vendor development tools, and other essential information for running on Theta. In addition, the hands-on collaborative sessions allowed teams to try out different code optimization approaches and experiment with the system's innovative memory hierarchy. Many projects were able to scale up to 2,000-3,000 nodes on Theta, with some taking advantage of the full system. Project team members presented many of these benchmarks, as well as other performance

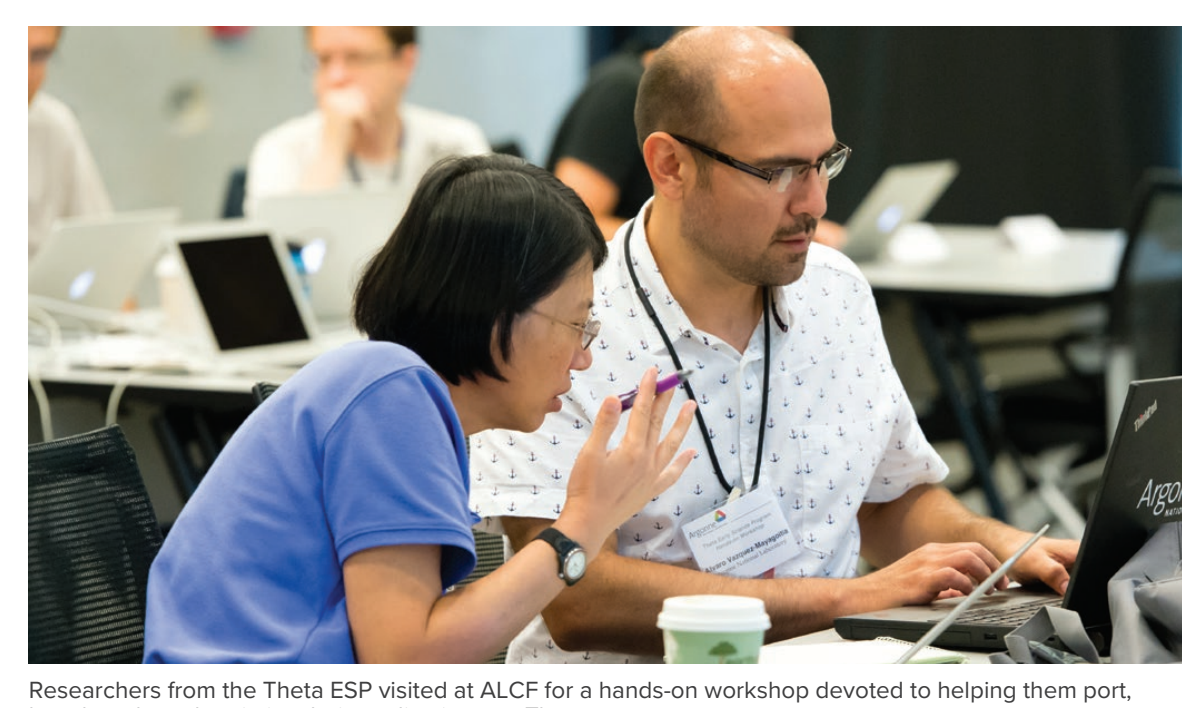

Researchers from the Theta ESP visited at ALCF for a hands-on workshop devoted to helping them port, benchmark, and optimize their applications on Theta. measurements, at the 2016 Intel ${ }^{\circledR}$ Xeon Phi' User's Group meeting held at Argonne in September.

Now that Theta is operational, ESP teams have begun running science simulations on the system to pursue their research goals, which range from modeling the brain to simulating the evolution of the universe. When the Early Science period is completed, each team will produce a technical report detailing their efforts to port and optimize their codes for Theta. They will present their results and lessons learned at a wrap-up workshop open to the entire ALCF user community. 


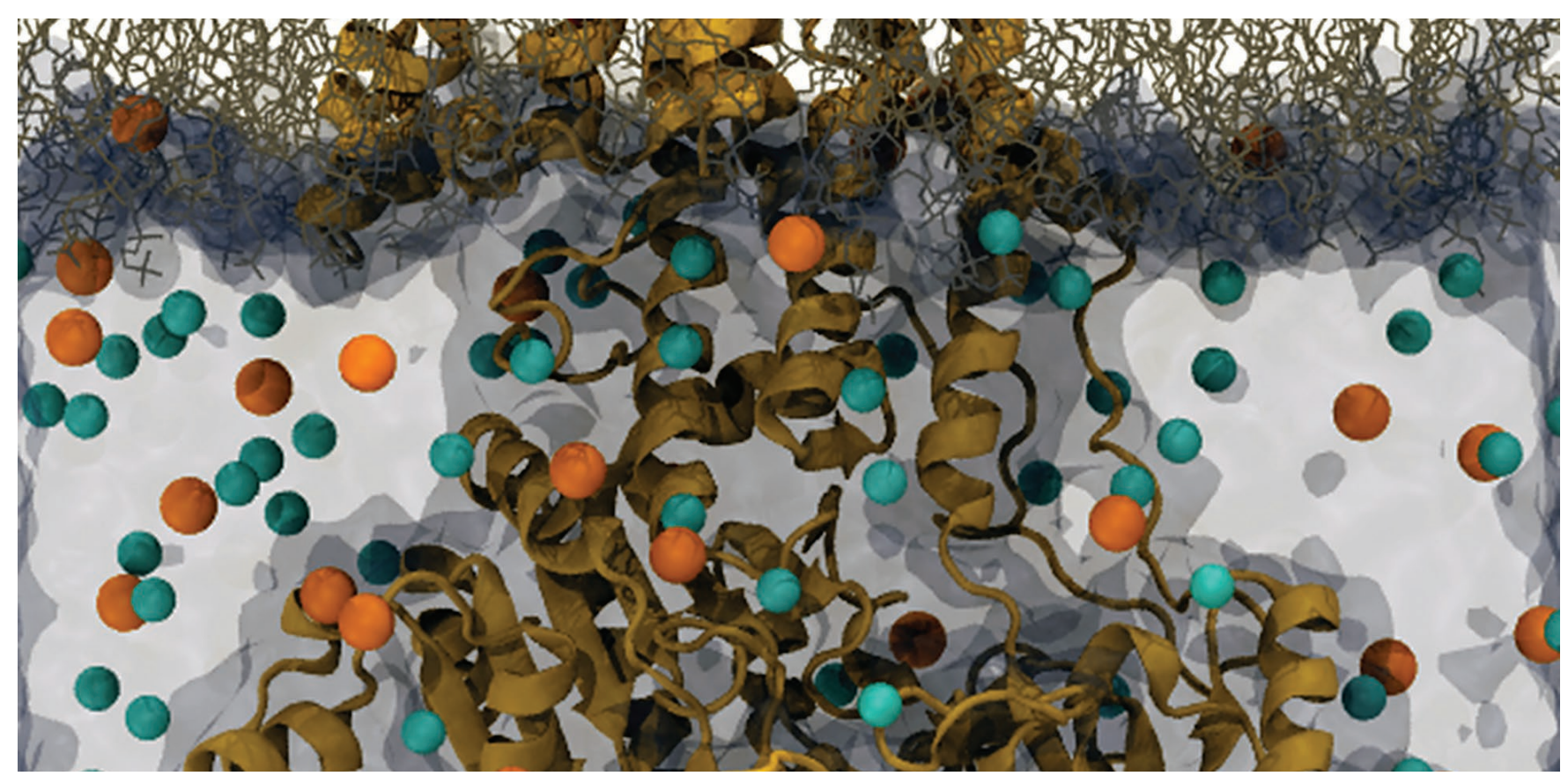

Benoît Roux's ESP project aims to determine the free energy landscapes of large membrane proteins, especially P-type ATPases such as the sodium-potassium pump (shown in yellow). Image credit: Brian Radak, Argonne National Laboratory/The University of Chicago; Huan Rui, The University of Chicago

\section{STRONG SCALING ON THETA}

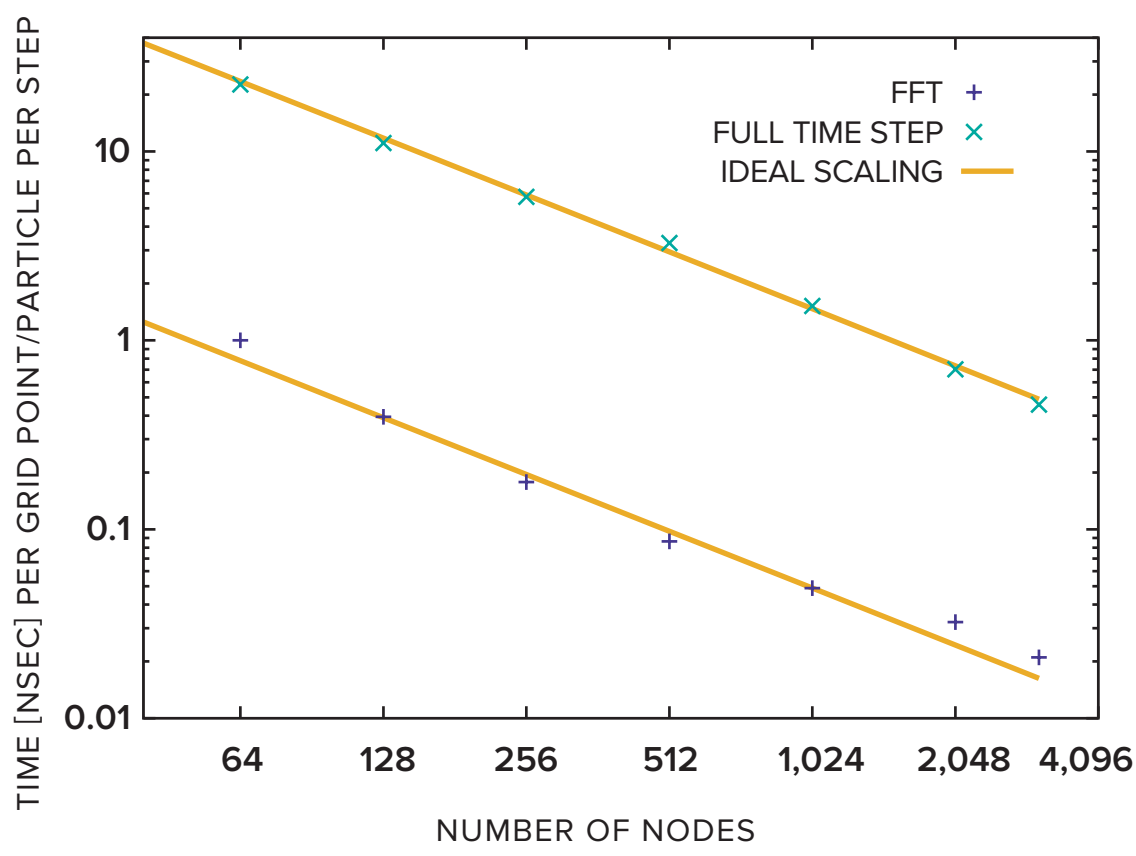

HACC strong scaling results on Theta for the entire application (indicated by teal " $x$ " symbols), and for just the fast Fourier transform (FFT) part of the application (indicated by purple pluses). The solid lines show ideal scaling. The tests were carried out on up to 3,072 nodes.

\section{EARLY RETURNS}

While the Early Science period does not hit full swing until 2017, several research teams have already achieved notable scaling and performance improvements on Theta.

$\square$ In initial results for the GFMC code, researchers demonstrated strong OpenMP thread scaling on a node, with the best throughput achieved using several MPI ranks with fewer threads each. Weak scaling was excellent up to 3,168 nodes.

$\square$ The HACC team found their application performed remarkably well in a strong scaling test, evolving $2,048^{3}$ particles in an $\left(800 \mathrm{~h}^{-1} \mathrm{Mpc}\right)^{3}$ volume (the target size for their hydrodynamics simulations in the Early Science period).

$\square$ In NAMD scaling tests using up to 3,072 nodes on Theta, researchers observed a $10 x$ speedup per node compared to the code's performance on Mira.

$\square$ For the Nek5000 code, researchers conducted a strong scaling test using a turbulent channel flow simulation. The code performed $7.2 x$ faster per node compared to its performance on Mira.

$\square$ Researchers performed a strong scaling study with PHASTA on an 80-billion element mesh. The case scaled perfectly to 192,000 cores on Theta, and fit into the system's MCDRAM with 1.2 million elements per core. 


\section{SHAPING THE FUTURE}

\section{BEYOND AURORA: THE PUSH TOWARD EXASCALE}

The ALCF is a key contributor to the DOE's Exascale Computing Project, a multi-laboratory initiative aimed at accelerating the development of a capable exascale computing ecosystem.

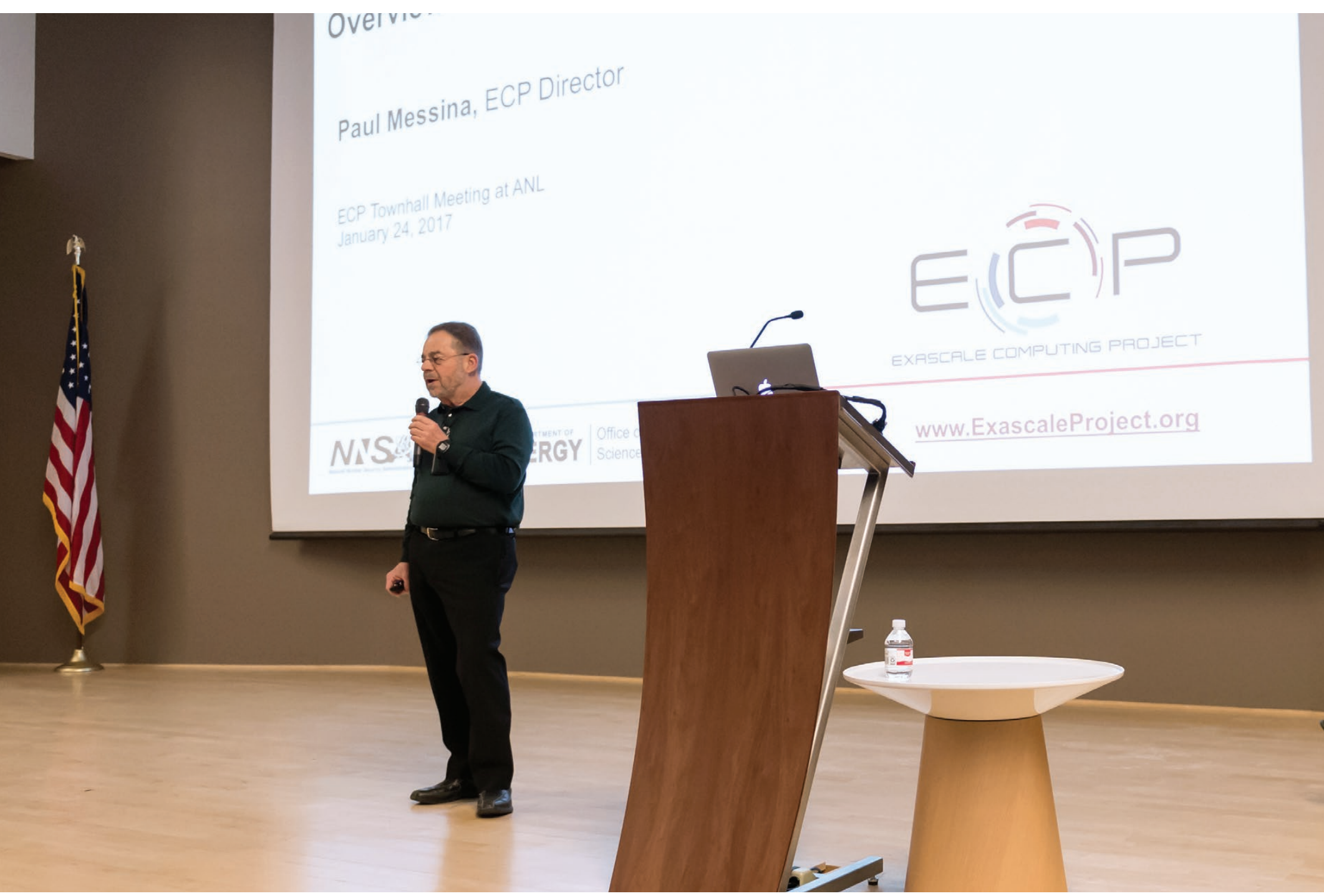


In addition to developing and operating some of today's most powerful computing systems, the ALCF is actively involved in the pursuit of future exascale systems, which will deliver approximately 50 times more performance than the top supercomputers currently in use.

From identifying the science capabilities needed from extremescale supercomputers to exploring and pioneering the technologies that will power them, the ALCF has long been a key player in the nation's efforts to develop a future exascale computing system.

In 2016, the DOE announced the launch of the Exascale Computing Project (ECP), a collaborative effort of two DOE organizations - the Office of Science and the National Nuclear Security Administration - that brings together multiple national laboratories to develop a productive exascale capability in the U.S. by 2021. Led by Paul Messina, Argonne Distinguished Fellow and former ALCF Director of Science, the ECP's mission is to build an ecosystem that encompasses applications, system software, hardware technologies, architectures, and workforce development to pave the way for the nation's first exascale systems.

ALCF experts and computing resources will play significant roles in helping the ECP achieve its goals. Several ALCF researchers are already engaged in ECP-funded projects in application development, software development, and hardware technology. The program will also support the operation of two additional Theta racks, allowing ECP researchers to use the ALCF's leading-edge system to pursue development work at a large scale.

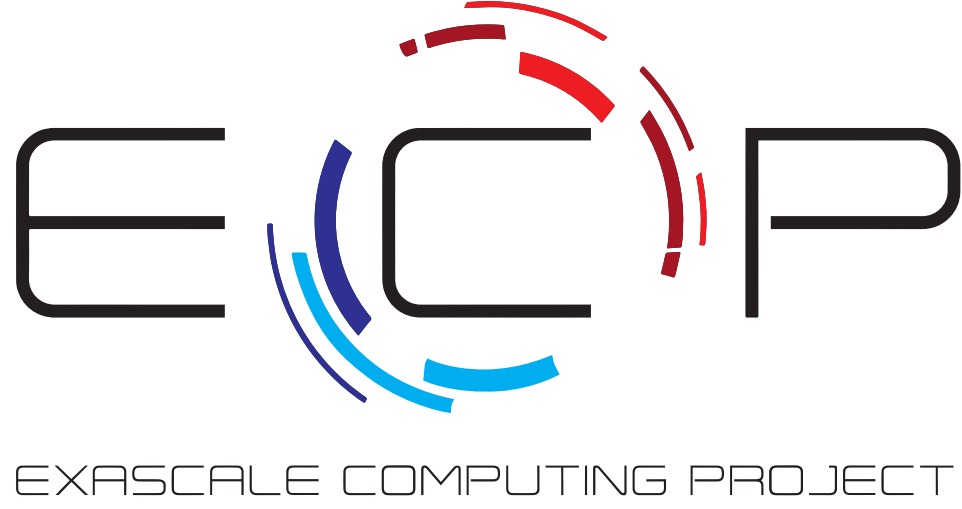

ACCELERATING EXASCALE

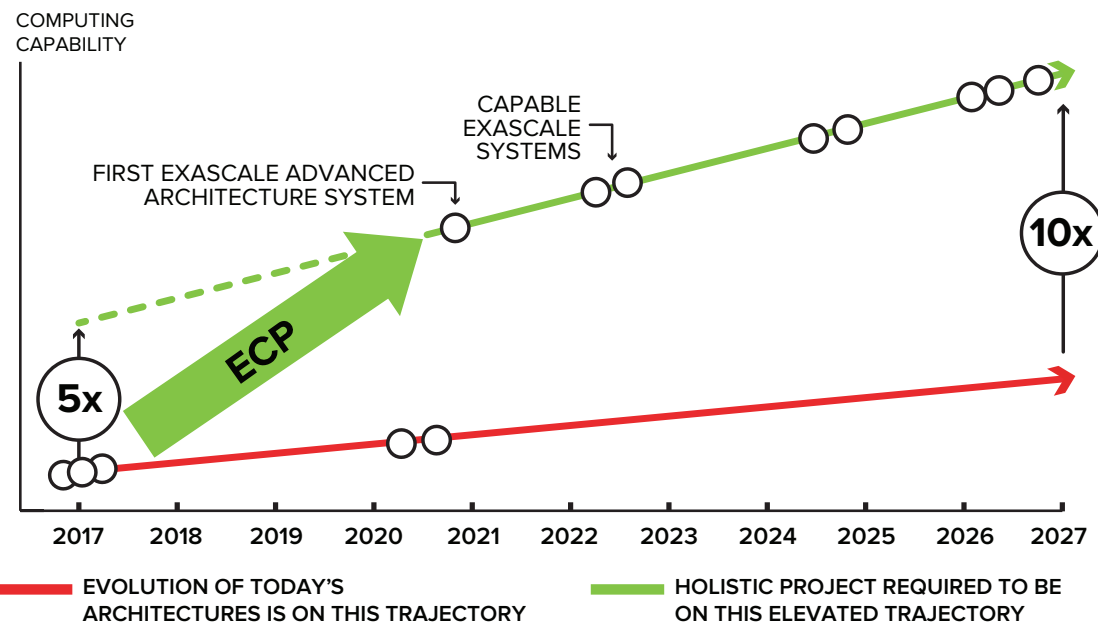

In the workforce development space, the ECP now funds the annual Argonne Training Program on Extreme-Scale Computing (ATPESC), which is organized and managed by ALCF staff. ATPESC is an intensive, two-week program that introduces attendees to the key skills and tools needed to carry out computational science and engineering research on leading-edge supercomputers and the extreme-scale systems of the future.

Ultimately, the ALCF and other DOE computing facilities will handle the procurement of future exascale-class supercomputers. Part of the ECP's role is to bridge and align the resources of DOE laboratories, allowing them to work more effectively with industry. This includes integration with technology and system vendors and software and application developers that goes beyond the specific needs and charters of any one laboratory. Prior to the procurement phase, the ECP will leverage its research and development work to establish the design, performance, and implementation requirements that will help shape the laboratories' exascale Request for Proposal documents. 


\section{SHAPING THE FUTURE}

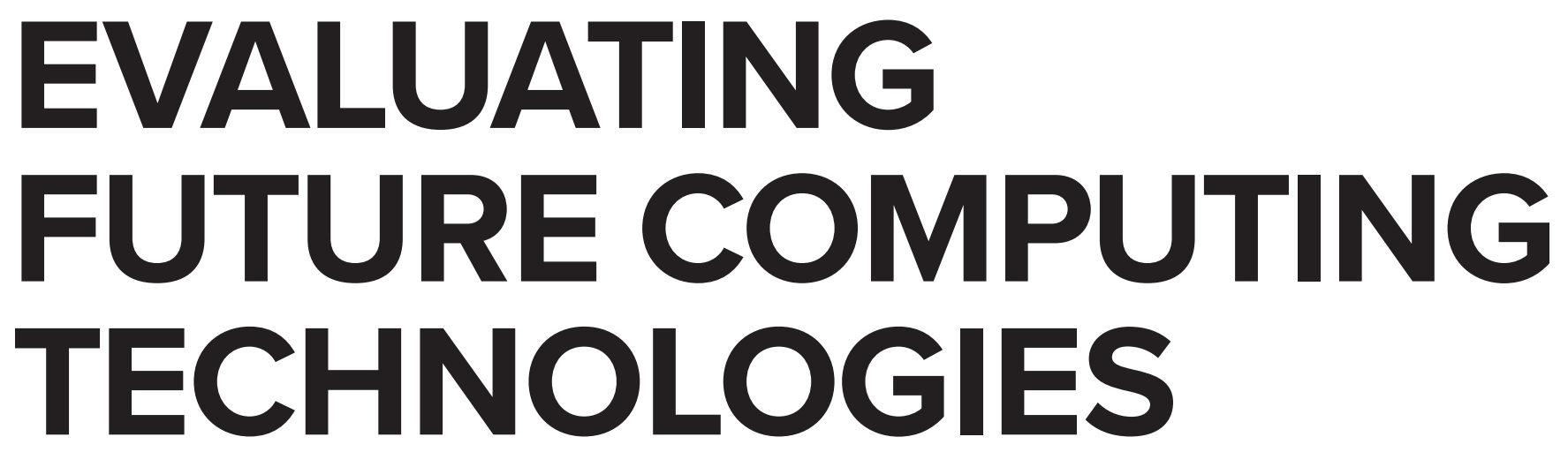

\section{Argonne's Joint Laboratory for System Evaluation (JLSE) is designed to help researchers assess and improve future extreme-scale computing technologies and systems of interest to the DOE.}

Established by the ALCF and Argonne's Mathematics and Computer Science Division, the JLSE was created to centralize Argonne's research activities aimed at evaluating future extreme-scale computing systems and technologies.

JLSE users from both divisions leverage existing infrastructure and next-generation hardware and software to explore low-level experimental computer and computational science, including operating systems, messaging, compilers, benchmarking, power measurements, $1 / O$, and new file systems.

By providing access to leading-edge computing resources and fostering collaborative research, the JLSE is allowing researchers to address Argonne and DOE needs in a variety of areas, including:

$\square$ Improving science productivity on future hardware and software platforms

$\square$ Providing an avenue for Argonne researchers to work collaboratively with HPC vendors on prototype technologies for petascale and beyond

$\square$ Investigating alternative approaches to current and future system deployments $\square$ Maintaining a range of hardware and software environments for testing research ideas

$\square$ Helping to drive standards on benchmarks, programming models, programming languages, memory technologies, etc.

For the ALCF, JLSE resources are enabling staff members to better prepare for the architecture of future systems. For example, ALCF researchers are using JLSE computing systems to analyze important application benchmarks and mini-apps to shed light on the performance and characteristics of next-generation platforms.

By considering architectural characteristics, such as instruction issue rates, memory and cache hierarchy, and vector instruction operations, the researchers are working to identify the capabilities and limitations of future architectures, best practices for improving the performance of applications, and, ultimately, a roadmap for users to effectively adapt and tune their codes for new leadership-class systems. 


\section{JLSE PROJECTS}

In 2016, the JLSE supported more than 75 Argonne users participating in over 30 projects. The following summaries represent a selection of current JLSE projects.

\begin{abstract}
ALCF Data Science Program Venkatram Vishwanath

Research teams from the ALCF Data

Science Program are using JLSE resources to explore and improve data science techniques, such as data mining, graph analytics, machine learning, and complex and interactive workflows.
\end{abstract}

\section{Evaluation of Neuromorphic Computing Hardware Hal Finkel, Fangfang Xia In collaboration with IBM, researchers \\ Argo is an exascale operating system and runtime stack being developed to support extreme-scale scientific computation.} are testing various use cases for neuromorphic hardware to develop an understanding of how to best train and make use of these artificial neural networks in hardware.

\section{Exascale Deep}

Learning-Enabled Precision Medicine for Cancer

\section{Rick Stevens}

Using the NVIDIA DGX-1 system and other JLSE computing resources, researchers are developing the CANcer Distributed Learning Environment (CANDLE), a computational framework designed to facilitate breakthroughs in the fight against cancer.

\section{Integration and Validation of Argo's Components \\ Swann Perarnau}

\section{Petrel Data Service Testbed Ian Foster, Michael E. Papka} Petrel is a pilot service for data management that allows researchers to store large-scale datasets and easily share that data with collaborators.

\section{RAN - RAM Area Network William E. Allcock}

Researchers are working with Kove, a high-performance storage vendor, to develop a network that treats RAM as a schedulable resource. This involves moving a portion of the RAM into a pool, so that it can be scheduled and better utilized.

\section{Testing Globus with Spectra Logic BlackPearl Ian Foster}

The project team is working with Spectra Logic to provide a turn-key research data storage solution by developing and testing a Globus interface for the vendor's BlackPearl object storage manager.

\section{Theta Early Science Program Tim Williams}

Theta Early Science Program teams used the Intel ${ }^{\circledR}$ Xeon Phi $^{\text {TM }}$ Knights Landing cluster and other JLSE resources to prepare and optimize applications for Theta in advance of the system being available.

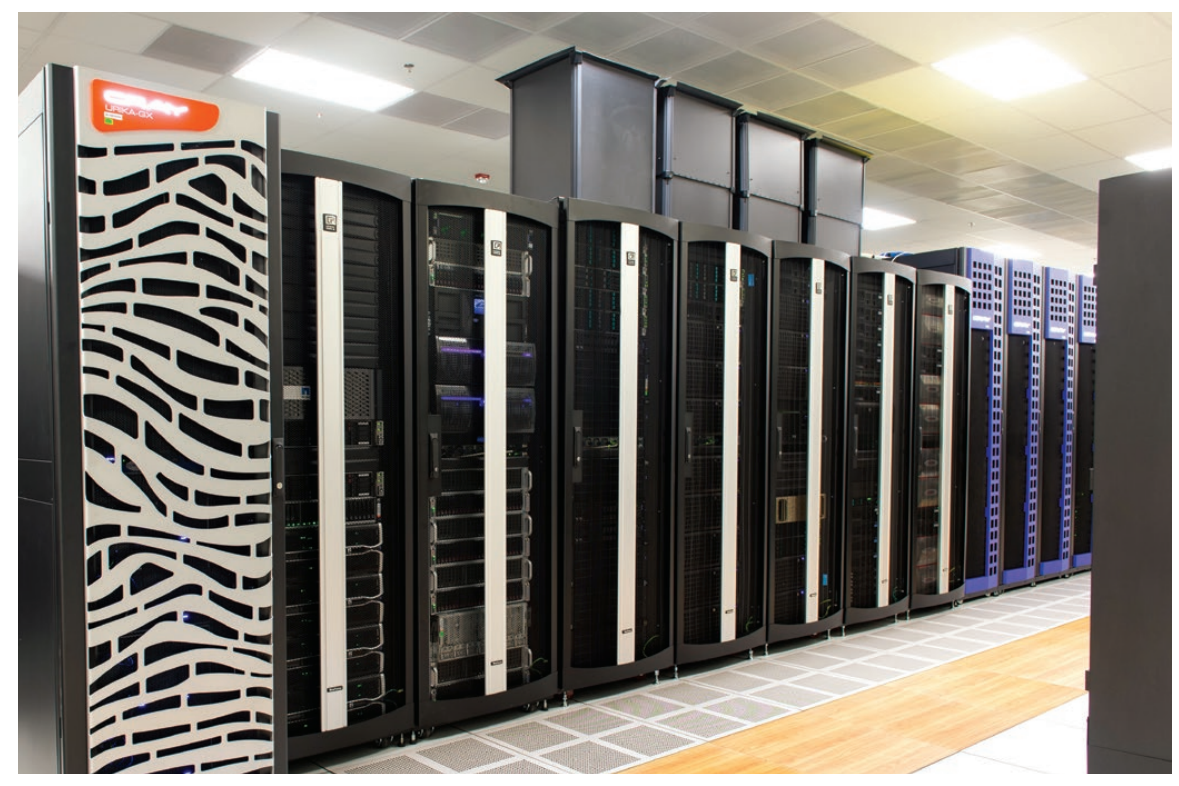

\section{JLSE TESTBEDS}

JLSE users' research and

development work is enabled

by the joint lab's diverse testbeds, which include:

$\square$ Intel ${ }^{\circledR}$ Xeon Phi ${ }^{\mathrm{Tm}}$ Knights Landing Cluster

$\square$ Cray Urika-GX Analytics Platform

$\square$ BM Power System S822LC

$\square$ AppliedMicro X-C1 Server Development Kit Plus

$\square$ NVIDIA DGX-1

$\square$ IBM Elastic Storage Server GL6 


\section{SHAPING THE FUTURE}

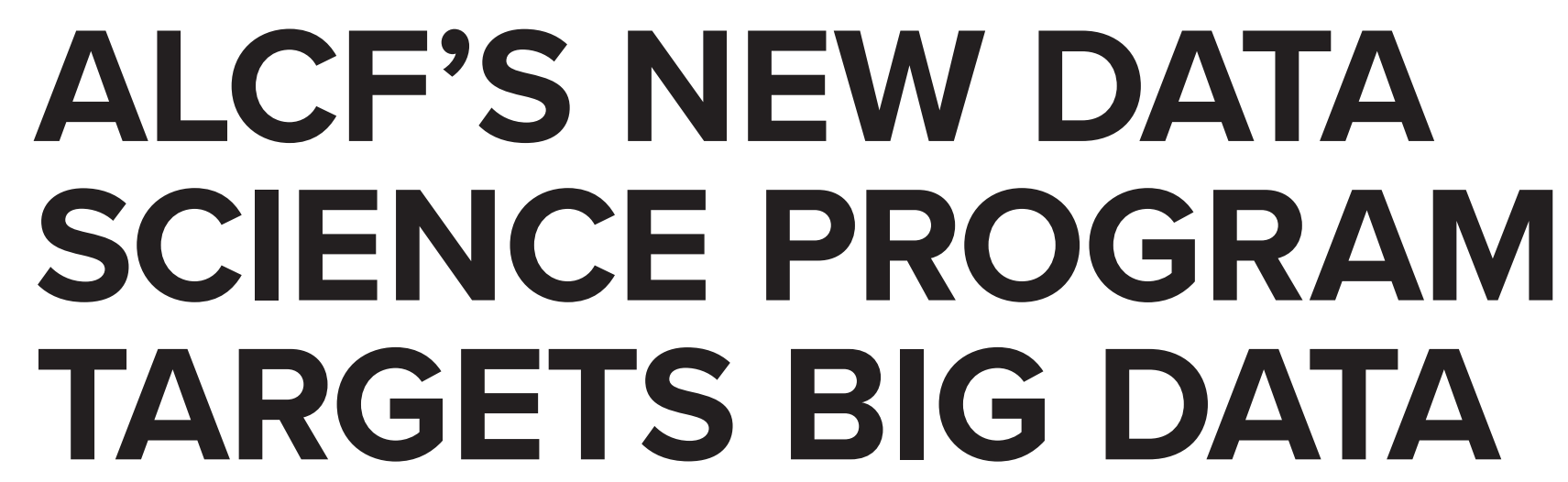

\section{With its pioneering Data Science Program, the ALCF is growing a community of users that can leverage leadership computing resources to advance data-driven discoveries.}

Large-scale experiments, metagenomics, urban planning, and many other data-centric application domains are generating increasingly larger amounts of data that contain scientifically significant discoveries. Leadership computing resources are massively powerful platforms that can be used to parse and analyze this data - provided the right tools and techniques can be developed, proved, and improved.

Launched in 2016, the ALCF Data Science Program (ADSP) was created to grow a community of users that are capable of using leadership-class supercomputers to advance data science across all scientific disciplines. The pioneering initiative is targeted at "big data" science problems that require the scale and performance of ALCF computing resources.

The program is allowing researchers to explore and improve a variety of computational methods and novel usage modalities that will help enable new data-driven discoveries. By developing and demonstrating rapid analysis techniques, such as data mining, graph analytics, and machine learning, together with workflows that will facilitate productive usage on extreme-scale computing systems, ADSP will help pave the way for more researchers to use supercomputers for their big data challenges in the future.

For the program's first year, the ALCF selected four projects that span experimental and computational sciences and range from physics to neuroscience. The projects, which have access to ALCF computing resources and staff, are split into two tiers, with the tier 1 projects receiving additional support from postdoctoral researchers. ADSP teams will be among the first to access Theta, the ALCF's new Intel-Cray system. Additional computing resources for this work include Mira, Cooley, and Sage, a Cray Urika-GX analytics platform housed in Argonne's Joint Laboratory for System Evaluation. 


\section{SHAPING THE FUTURE}

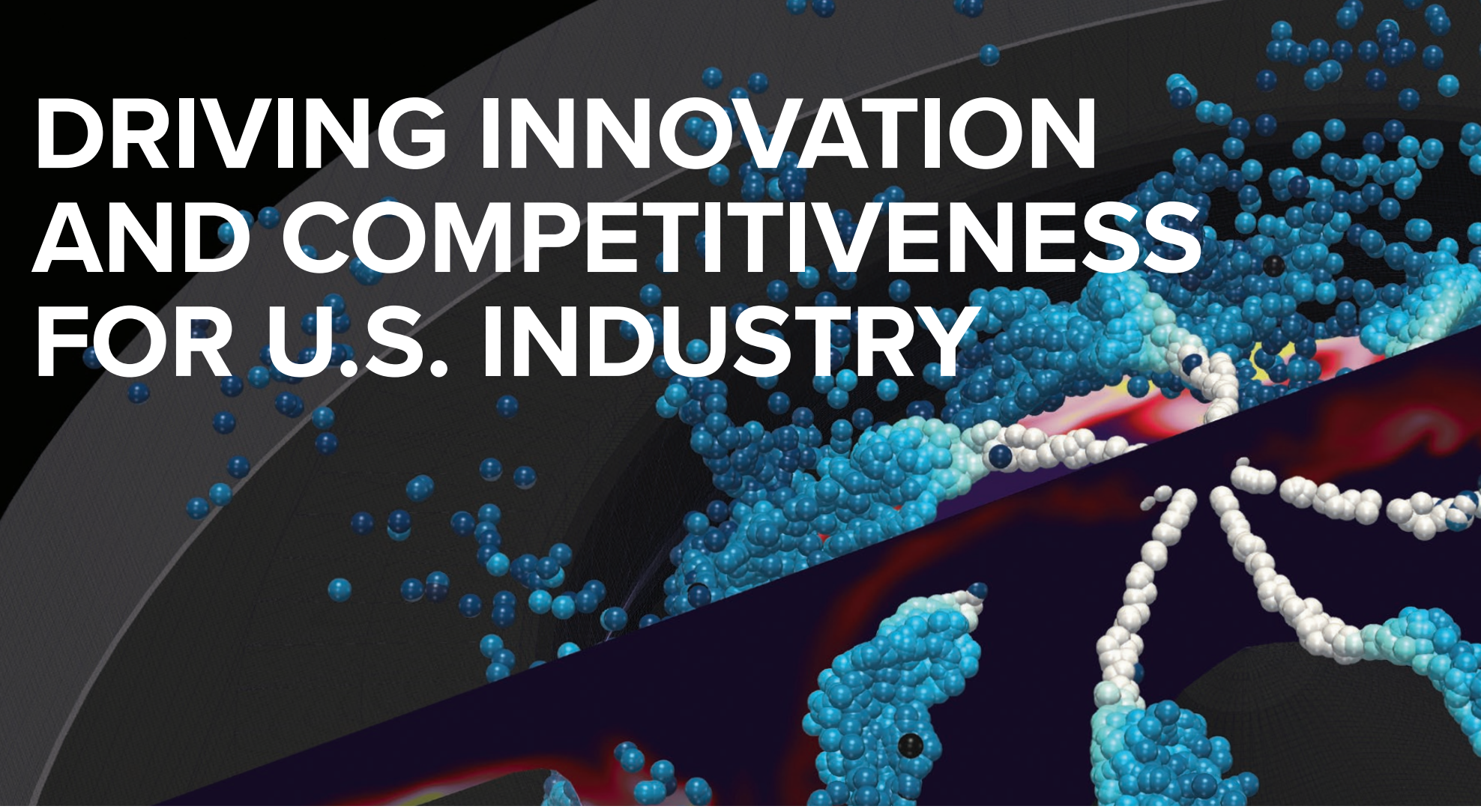

Industry partnerships with the ALCF help to strengthen the nation's innovation infrastructure and expand the use of supercomputing resources for national competitiveness.

The ALCF's industrial outreach efforts aim to grow the facility's base of industry users by identifying prospective companies of all sizes, from start-ups to Fortune 500 corporations, that could benefit from leadership computing resources and collaborative opportunities with the ALCF and across Argonne.

Supercomputers like Mira enable U.S. companies to tackle computational problems that are impossible to address on most internal computing systems. Access to ALCF computing systems gives them the ability to perform more complex simulations, achieve more accurate predictions, and create higher fidelity models of everything from battery materials to combustion.

The results allow companies to accelerate critical breakthroughs, verify uncertainties, and drastically reduce or eliminate the need to build multiple prototypes. Ultimately, industry partnerships with the ALCF help to strengthen the nation's

innovation infrastructure and expand the use of HPC resources for national competitiveness.

In addition to gaining access to some of the world's most powerful supercomputers, industry users also benefit from the ALCF's expertise in novel computational methods and algorithms, application porting, performance tuning and scaling, and data analysis and visualization.

The ALCF has recently enhanced its industry outreach program by partnering with other Argonne user facilities and divisions, including the Technology Development and Commercialization Division. This collaborative approach allows the ALCF to present a more complete picture of the laboratory's resources and encourages additional collaborations. These efforts have resulted in broader engagements across the laboratory with a number of companies. 
High-fidelity 3D simulations provide unique insights into the development of next-generation engines. This simulation captures fuel distribution inside the combustion chamber of a GM $1.9 \mathrm{~L}$ diesel engine being operated in a novel low-temperature combustion mode developed at Argonne.

Image credit: Joseph A. Insley, Janardhan Kodavasal, and Sibendu Som, Argonne National Laboratory

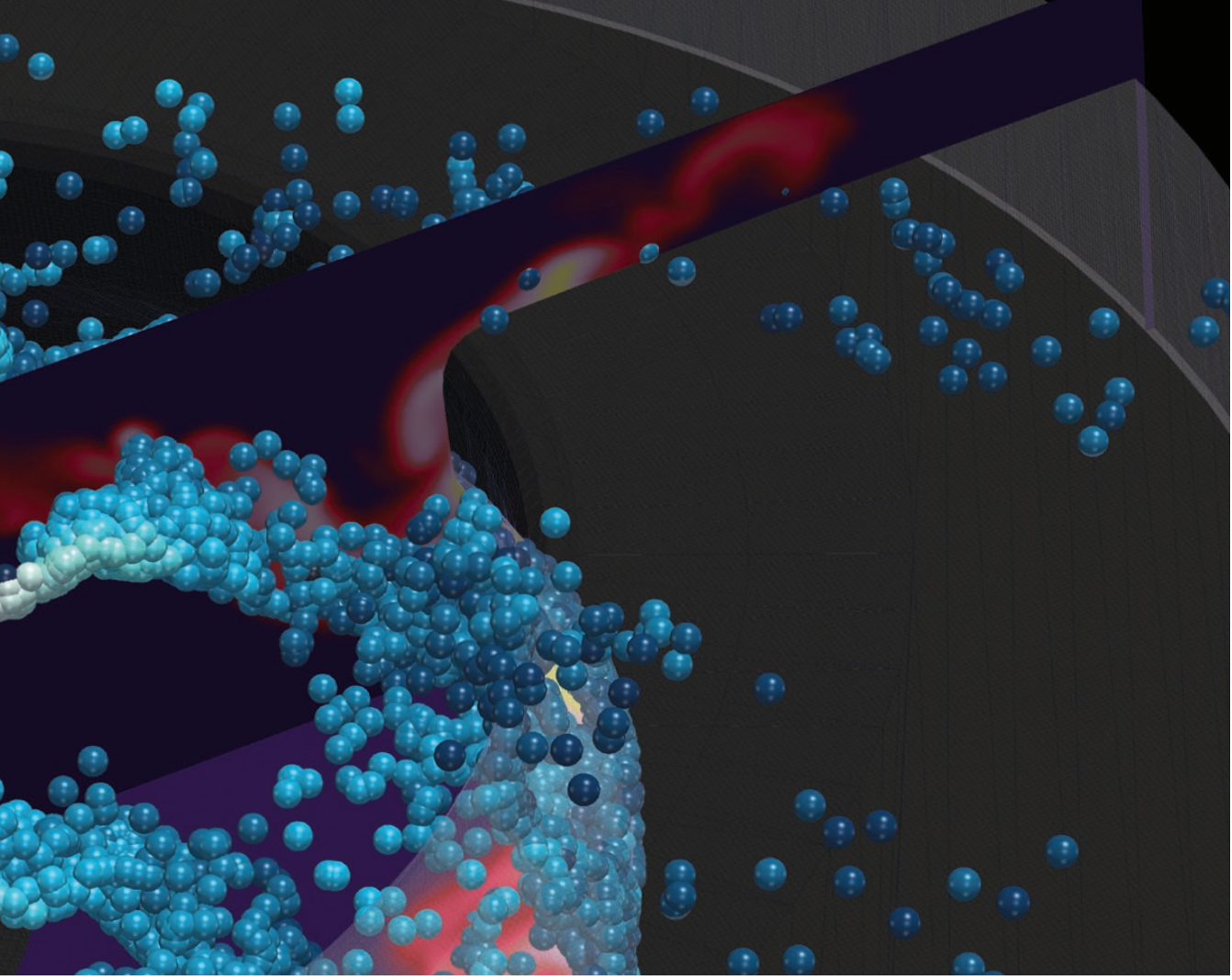

\section{Brewer Science Seeks to Develop New Materials for Semiconductor Industry \\ Brewer Science brings innovative \\ Convergent Science Uses Supercomputers to Improve Virtual Engine Design} material technologies, process solutions, manufacturing, and metrology capabilities to advance various industries such as

semiconductor, electronics packaging, and printed electronics. The company formed a multifaceted partnership with the ALCF and other Argonne divisions to advance their work with new material platforms, enabling multilayer lithography patterning and directed self-assembly applications. Brewer Science's ultimate goal is to use supercomputers to better understand the codependence of structure-property relationships of new polymer-based compositions, providing insights to guide the development of materials that will benefit the semiconductor industry.
Researchers from Argonne's Virtual Engine Research Institute and Fuels Initiative (VERIFI) are working with ALCF staff and Convergent Science Inc. to optimize the CONVERGE code, a commercial computational fluid dynamics (CFD) software tool, for Mira. This ongoing collaboration aims to develop HPC engine modeling and simulation capabilities, providing U.S. automotive manufacturers with a cost-effective tool to accelerate the development of more energyefficient engines. Such tools have the potential to improve the fuel economy of vehicles, thereby reducing U.S. dependence on foreign oil and reducing carbon emissions.

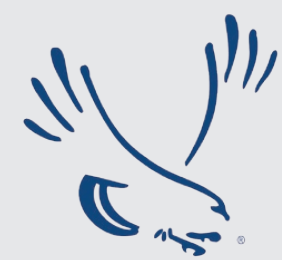

\section{brewer science}
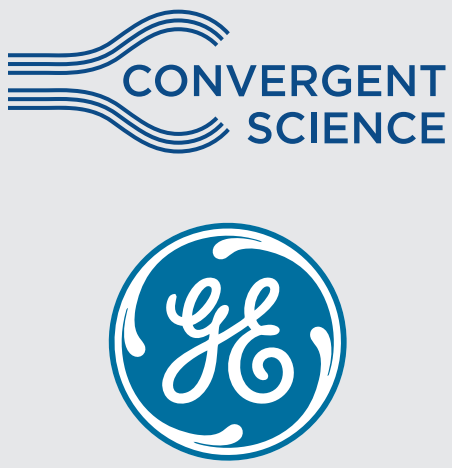

\section{GE Performs Simulations to Guide the Design of Better Aircraft Engines}

The aviation industry demands each generation of engines powering modern aircraft improve fuel efficiency while meeting environmental regulations. Pursuing insights toward better designs, GE researchers, in partnership with Cascade Technologies, are using ALCF supercomputers to study the complex physics of an engine's combustor liner flows. Findings from this project will provide high-fidelity datasets to guide the low-fidelity models currently available to engine designers. Ultimately, the results will help engineers design and optimize competitive aircraft engines that deliver better fuel efficiency, lower emissions, and improved performance. 


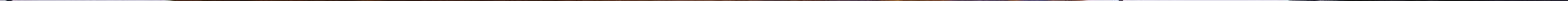


DP

\section{COMMUNITY \& OUTREACH}

As a leader in the HPC community, the ALCF is actively involved in efforts to broaden the impact of supercomputers and grow the community of researchers who can use them for computational science and engineering research. 


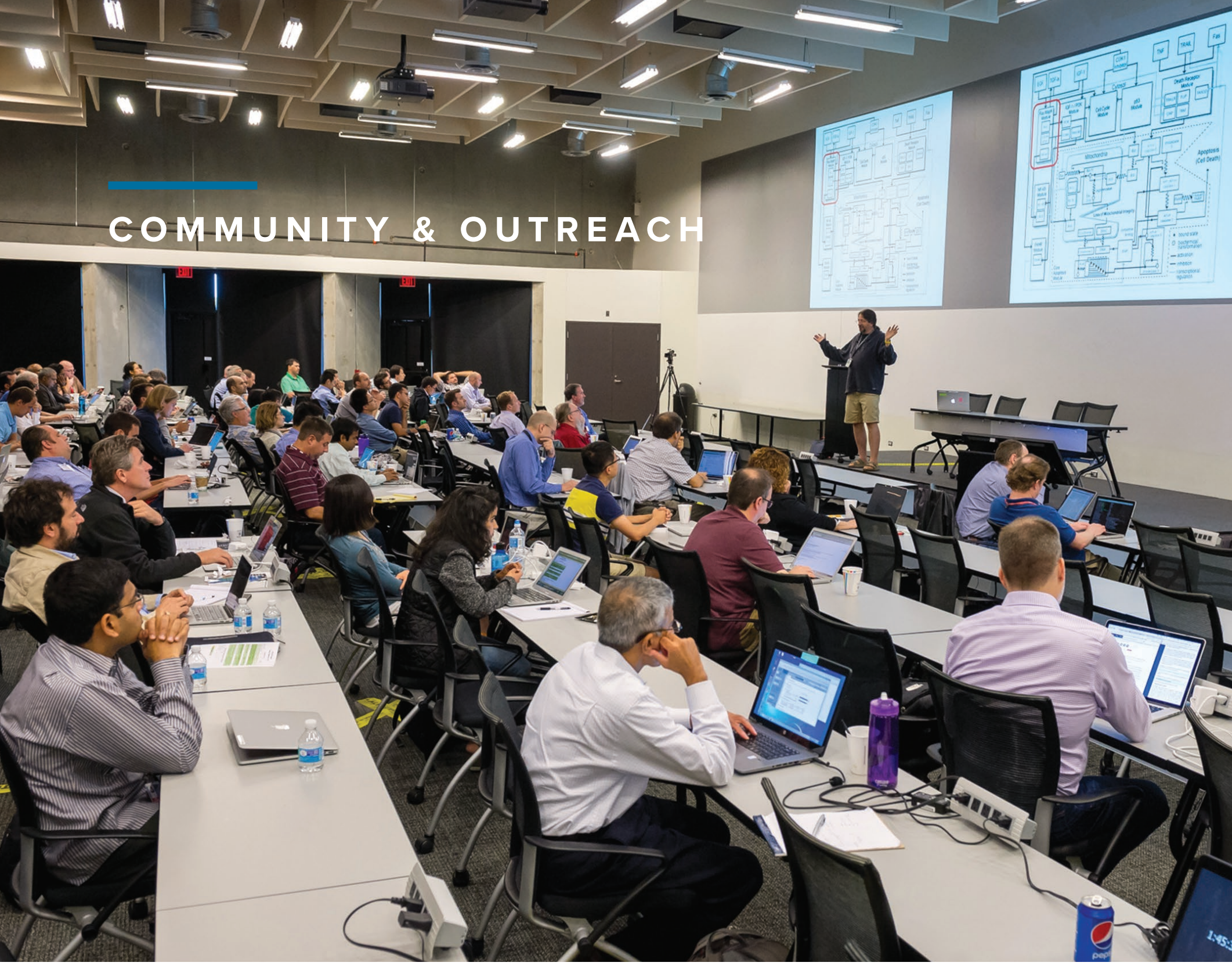

Argonne Associate Laboratory Director Rick Stevens addresses researchers at the 2016 Intel ${ }^{\circledR}$ Xeon Phi" User's Group (IXPUG) annual meeting.

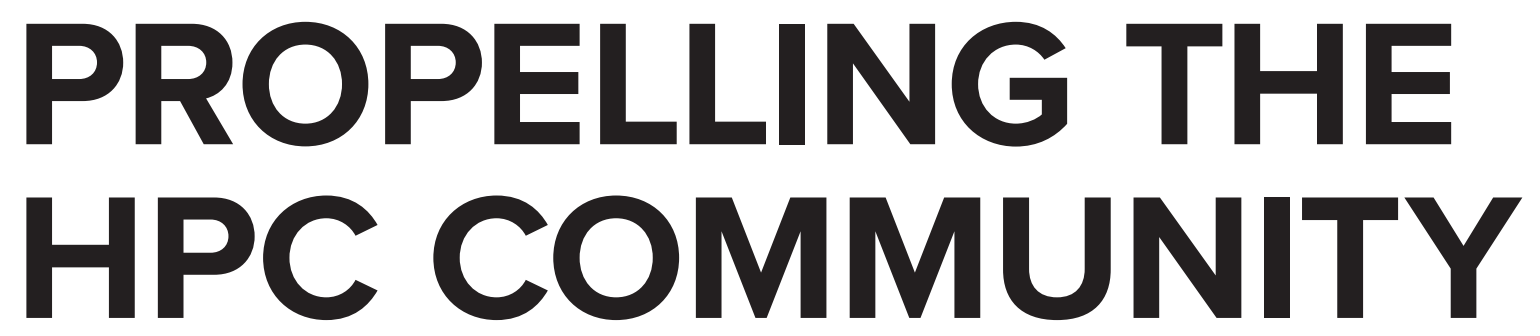

ALCF researchers are engaged in many forward-looking activities aimed at advancing the use of supercomputers for discovery and innovation. 


\section{Accelerating the Search for New Physics}

Physicists from across the country visited Argonne National Laboratory in April for a two-day workshop designed to generate new ideas and projects that will advance breakthroughs in physics. Sponsored by the ALCF and Lawrence Livermore National Laboratory, the Lattice for Beyond the Standard Model Physics 2016 workshop brought together the computational, phenomenological, and experimental communities to present recent findings and explore opportunities for collaboration. The focus of this year's workshop was on the role that lattice numerical simulations can play in the search for physics beyond the Standard Model.

\section{Advancing the QMC Method}

The quantum Monte Carlo (QMC) method is one of the most accurate electronic structure methods, providing an important computational tool for performing many-body calculations for a broad range of electronic systems. In 2016, the ALCF was named an Intel Parallel Computing Center for QMCPACK, a high-performance, open-source QMC code. Bringing together researchers from ALCF, Sandia National Laboratories, and Intel, the center is focused on optimizing QMCPACK for the next-generation Intel ${ }^{\circledR}$ Xeon $\mathrm{Phi}^{\mathrm{TM}}$ computing architectures that will be going online at several DOE supercomputing centers in the coming years. In a separate effort to advance QMC research, ALCF staff and computing resources supported the 2016 QMC Workshop, a weeklong, hands-on training event at the University of Illinois at UrbanaChampaign that introduced attendees to the fundamentals of QMC theory, and recent developments in methods and applications in physics, chemistry, and materials sciences.

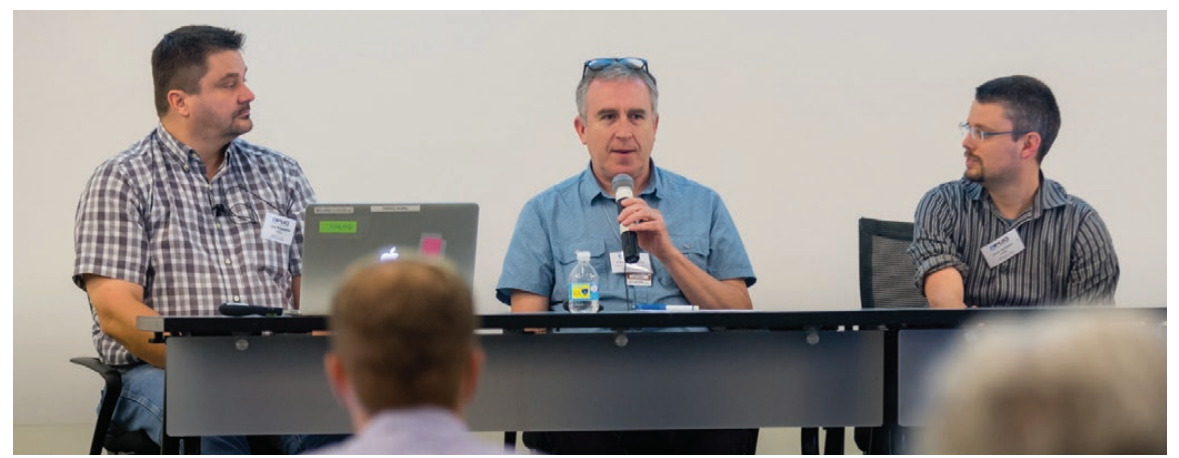

ALCF Deputy Director of Science Tim Williams (center) participates in a panel discussion on earlyexperiences with the Intel ${ }^{\circledR}$ Xeon $\mathrm{Phi}^{\mathrm{m}}$ processor at the 2016 IXPUG annual meeting.

\section{Identifying DOE Requirements for Exascale}

For the past two years, the ALCF has collaborated with its fellow DOE ASCR facilities to conduct a series of Exascale Requirements Reviews to determine the mission-critical computational science objectives for each of the six DOE Office of Science program offices through 2025. These workshops brought together key domain scientists and computational science experts to identify the requirements for developing an exascale ecosystem that includes computation, data analysis, software, workflows, HPC services, and other features. ALCF staff members, in collaboration with Argonne's Communications, Education, and Public Affairs Division, were also responsible for managing and producing the final reports from each of the six reviews, as well as developing and maintaining the Exascale Requirements Reviews website (exascale.org).

\section{Illuminating the Intel Xeon Phi Processor}

In September, Argonne National Laboratory hosted the Intel ${ }^{\circledR}$ Xeon Phi $^{\text {im }}$ User's Group's (IXPUG) 2016 annual meeting, which welcomed more than 130 researchers from around the world for four days of tutorials, workshops, and talks on the Intel ${ }^{\circledR}$ Xeon $\mathrm{Phi}^{\mathrm{TM}}$ processor. The ALCF's next-generation supercomputers, Aurora and Theta, are based on successive generations of the Intel processor. As one of the founding members of IXPUG, the ALCF has been active in the group's mission to provide a forum for exchanging information to enhance the usability and efficiency of scientific and technical applications running on large-scale HPC systems that use the new processor.

\section{Showcasing HPC Capabilities for Engine R\&D}

As a partner in Argonne's Virtual Engine Research Institute and Fuels Initiative (VERIFI), the ALCF played a key role in VERIFI's 2016 workshop on the role of simulations and high-performance computing in engines and fuels co-optimization. Held at the laboratory in June, the two-day workshop welcomed several representatives from the automotive industry for guidance on how VERIFI and the ALCF can help accelerate $R \& D$ efforts related to next-generation engines and fuels. In addition to showcasing current methodologies and capabilities, ALCF staff facilitated a hands-on session with Mira to demonstrate how supercomputers can be used to quickly run a series of practical engine simulations. 


\section{COMMUNITY \& OUTREACH}

The ALCF is committed to providing training and outreach opportunities that prepare researchers to efficiently use its leadership computing systems, while also cultivating a diverse and skilled HPC workforce for the future.

\section{ENGAGING CURRENT AND FUTURE USERS}

The 2016 Argonne Training Program on Extreme-Scale Computing brought together 65 participants for two weeks of instruction on the tools and approaches needed to use the world's most powerful supercomputers for science and engineering research.

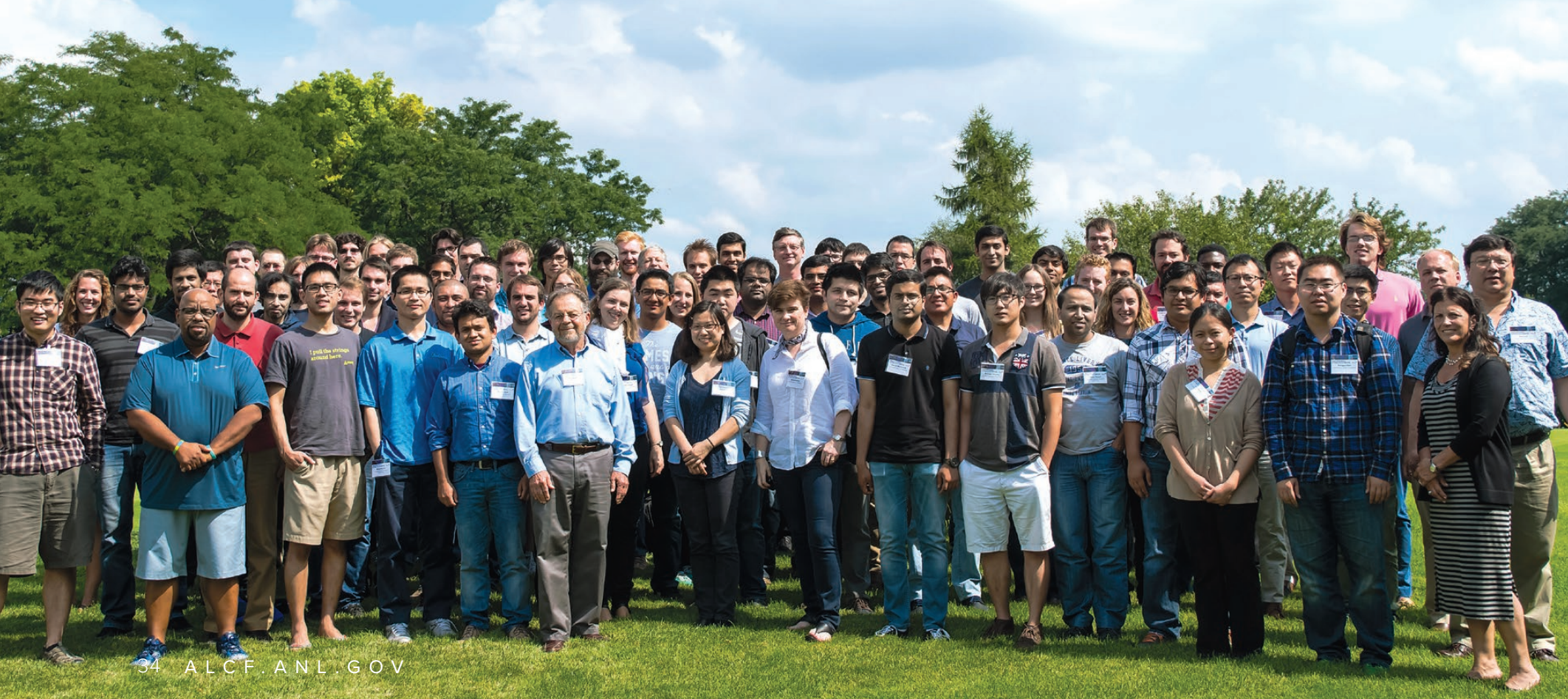




\section{INSPIRING STUDENTS}

\section{Computational Science Coursework}

ALCF staff played a large role in organizing and executing the Harvard University graduate course Extreme Computing: Project-Based High Performance Distributed and Parallel Systems. This applied computation course, part of a category of courses developed by Harvard's Institute of Applied Computational Science, highlighted the use of supercomputers to solve scientific problems and provided hands-on lessons, which included running jobs on Mira.

\section{Hour of Code}

As part of Code.org's annual Hour of Code event in December, several ALCF staff members visited Chicago-area schools to spark interest in computer science and coding. Working with classrooms ranging from kindergarten to high school, the ALCF volunteers led a variety of activities designed to demystify code and show that anybody can learn the basics. The global outreach campaign aims to expand participation and increase diversity in computer science.

\section{Promoting STEM Careers to Young Women}

Through participation in Argonne events like Introduce a Girl to Engineering Day and the Science Careers in Search of Women conference, ALCF staff members have the opportunity to connect with young women and introduce them to potential career paths in science, technology, engineering, and mathematics (STEM). The ALCF also promotes STEM careers to women

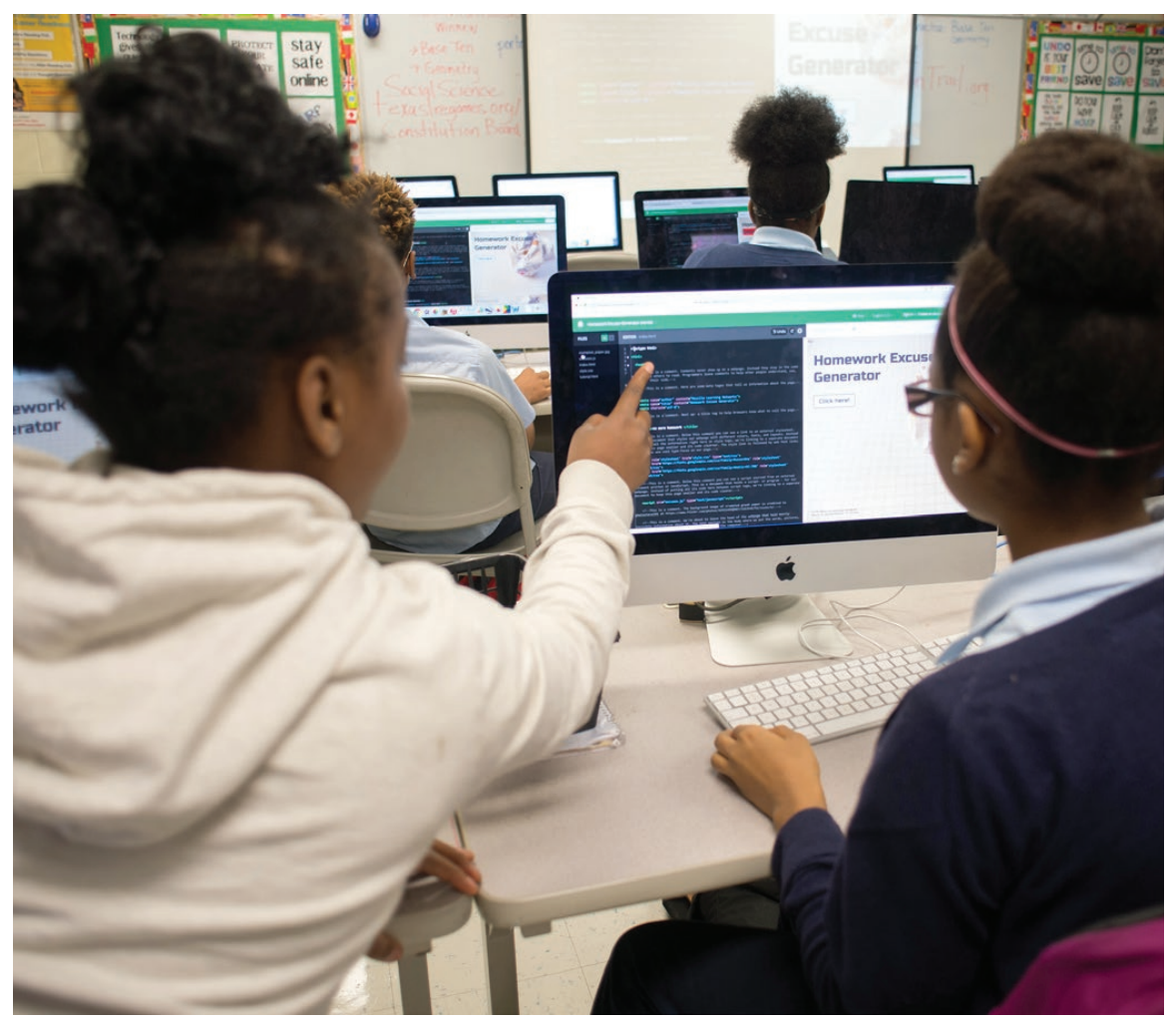

Several ALCF staff members volunteered to lead Hour of Code activities in K-12 classrooms as part of Computer Science Education Week in December.

through contributions to Argonne's Women in Science and Technology group, the Anita Borg Institute, the Grace Hopper Celebration of Women in Computing, and the Rocky Mountain Celebration of Women in Computing.

\section{Summer Coding Camp}

For the past two years, ALCF staff members have teamed with Argonne's education office to host an intensive coding camp for Chicago-area high school students. The weeklong course, developed and delivered by ALCF computer scientists, emphasized problem-solving skills and hands-on activities, including coding in Python and using a Raspberry Pi to program a robot.

\section{Summer Student Program}

In 2016, 22 students from universities around the country spent their summer at the ALCF, tackling a wide variety of projects that covered everything from HPC system administration and data analytics to computational science and performance engineering. Every year, the facility solicits student project proposals from staff members who are interested in mentoring summer students. Through programs like DOE's Science Undergraduate Laboratory Internship program and Argonne's Research Aide Appointments, college students, ranging from freshmen to $\mathrm{PhD}$ candidates, are brought in to work alongside ALCF mentors on real-world research projects. The program culminates with a special symposium in which the students present their project results. 


\section{COMMUNITY \& OUTREACH}

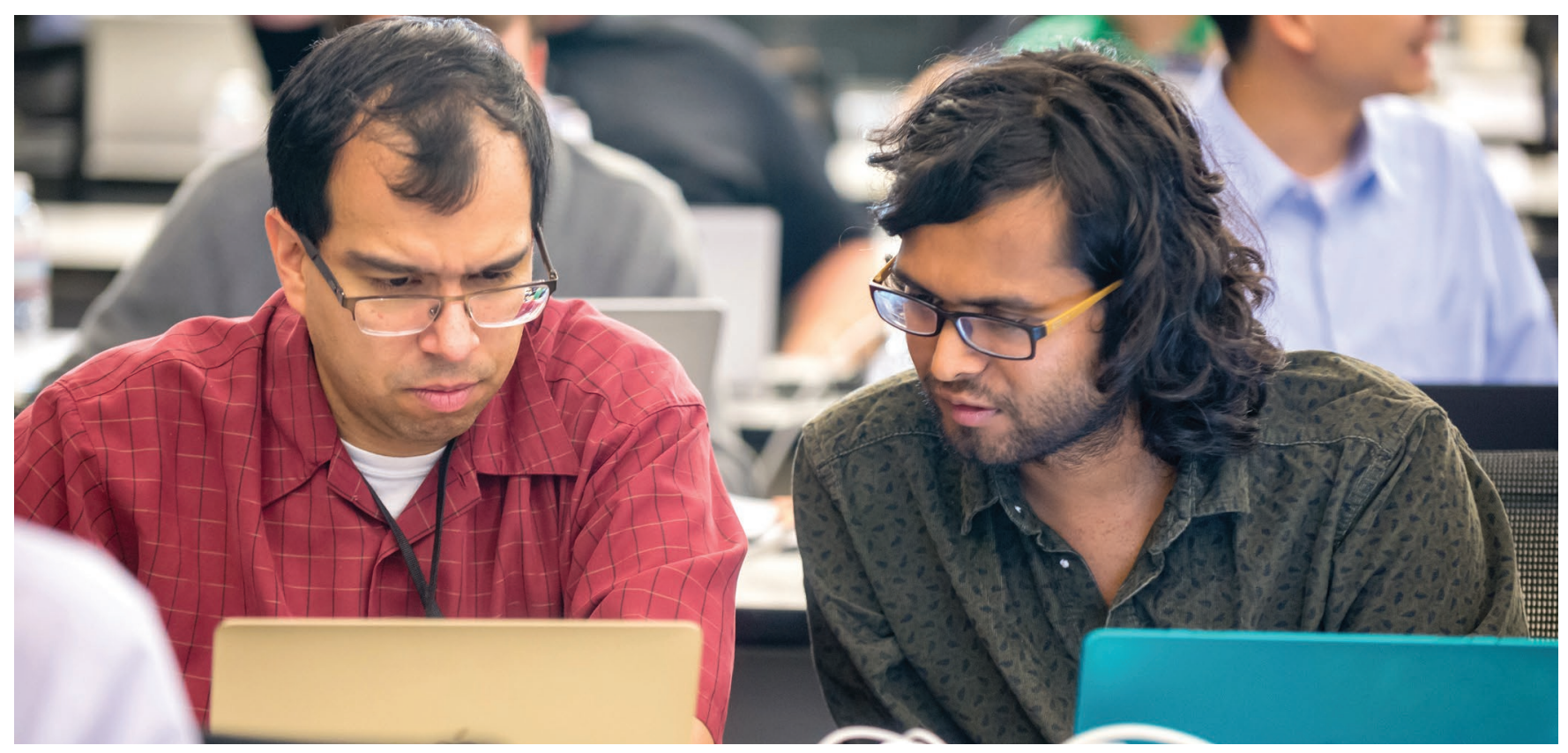

The ALCF's annual hands-on workshop provides an opportunity for users to work directly with ALCF staff to test, debug, and improve their codes.

\section{TRAINING USERS}

\section{Annual Hands-On Workshop}

In May, the ALCF's annual hands-on workshop, Scaling Your Science on Mira, welcomed both prospective and current users to work directly with ALCF computational scientists and industry experts on testing, debugging, and improving their codes on the facility's IBM Blue Gene/Q supercomputer. One of the workshop's goals is to help researchers demonstrate code scalability for a future allocation award at the ALCF. The two primary allocation programs for DOE leadership computing resources INCITE and ALCC - require project proposals to convey both scientific merit and computational readiness. The ALCF's hands-on workshop is a key resource for many teams seeking to fulfill the latter requirement. Current
ALCF users also attend the annual workshop to learn tips and techniques for maximizing their time on the facility's systems. In addition to hands-on sessions, the event featured talks on the IBM Blue Gene/Q architecture, parallel I/O, data analysis, and various HPC tools.

\section{ATPESC}

Now in its fourth year, the Argonne Training Program on Extreme-Scale Computing (ATPESC) is designed to teach participants the key skills and tools needed to efficiently use leading-edge supercomputers. Developed and managed by ALCF staff, the rigorous training program brought 65 graduates, postdocs, and researchers together at the Pheasant Run Resort in St. Charles, Illinois, in August, for two weeks of instruction on HPC codes, software, and architecture. As with previous years, all of the 2016 ATPESC presentations were recorded and posted to YouTube to extend the reach of the program. ATPESC is now funded by the Exascale Computing Project, a collaborative effort of the DOE Office of Science and the National Nuclear Security Administration.

\section{Best Practices for HPC Software Developers}

In 2016, the ALCF, the Oak Ridge Leadership Computing Facility, the National Energy Research Scientific Computing Center, and the Interoperable Design of ExtremeScale Application Software (IDEAS) project launched a series of webinars - Best Practices for HPC Software Developers - to help users of HPC systems carry out their software development more productively. 


\section{Cray Urika-GX Training}

With the launch of a Cray Urika-GX system this fall, Argonne National Laboratory became the first research center to deploy the new computing platform for big data analytics. In December, a group of 38 researchers gathered at the laboratory to learn how the system, named Sage, can help advance their data-intensive science problems. Hosted by the ALCF, the two-day workshop, led by experts from Cray, provided an overview of the new system's features and capabilities, and an opportunity for collaborative, hands-on training sessions. Sage, which is housed in Argonne's Joint Laboratory for System Evaluation, is one of the computing resources available to researchers participating in the ALCF Data Science Program.

\section{Theta ESP Workshop}

In August, the ALCF hosted researchers from its Theta Early Science Program (ESP) for a hands-on workshop to help them port, benchmark, and optimize their applications on the facility's next-generation Intel-Cray system. The four-day workshop included instruction from ALCF, Intel, and Cray staff on various tools and tips for running on Theta, and collaborative sessions aimed at getting the research teams' codes running on the actual machine.

\section{Virtual Training}

Employing an interactive videoconference format, the ALCF is able to connect with users from around the globe to provide virtual training on ALCF services and resources. Offered several times a year, the Getting Started on ALCF

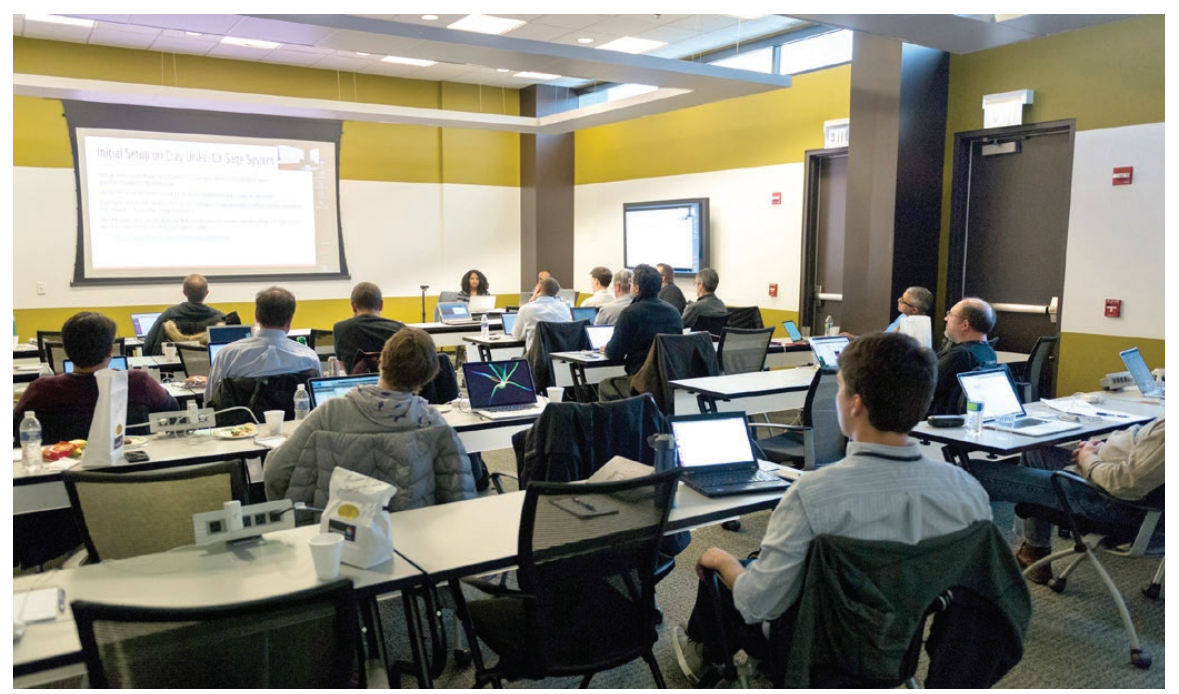

In December, the ALCF hosted a two-day workshop to introduce a group of researchers to Sage, Argonne's new Cray Urika-GX platform.

\section{From introducing students to exciting career possibilities in} HPC to working with users to inform them of new approaches for leadership-class systems, education is a critical part of the ALCF's mission.

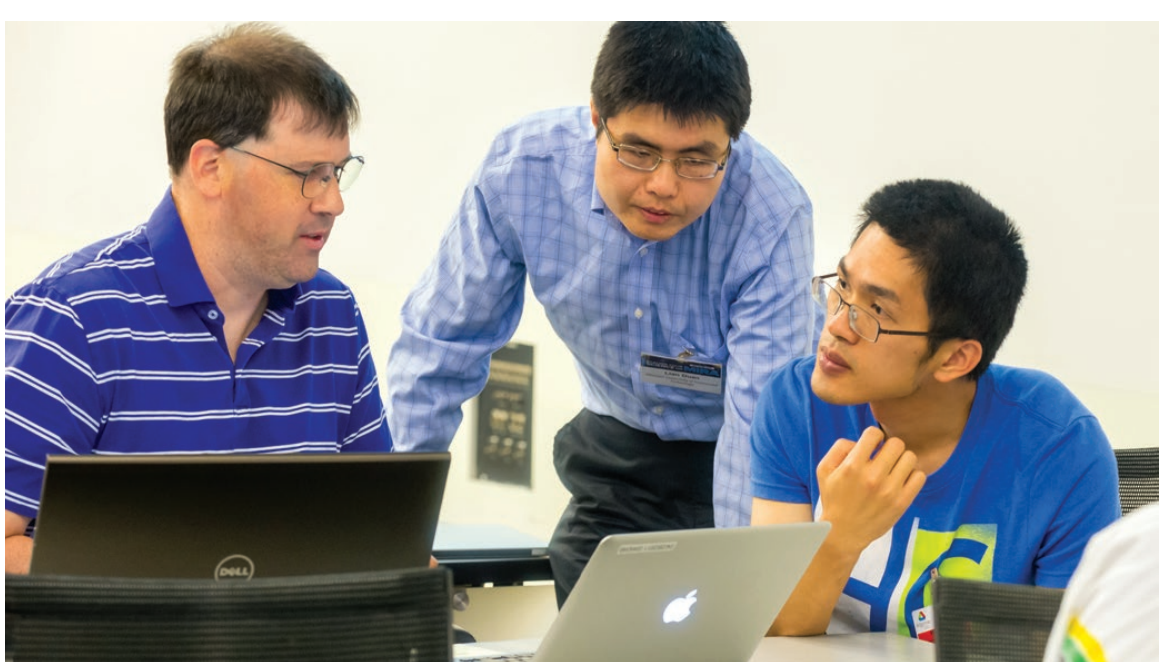

The ALCF's hands-on workshop is a key resource for researchers seeking to demonstrate code scalability for project proposals.

Resources videoconference is targeted at new users and those in need of a refresher. The highly interactive sessions cover the basics that researchers need to get their projects up and running, and give users an opportunity to receive guided assistance in building their codes and submitting jobs on Mira.
The ALCF also uses the virtual format for its Ensemble Jobs for Better Throughput videoconference. This specialized training session provides hands-on experience setting up an ensemble job script and helps users identify which job submission type is best for their projects. 


\section{SCIENCE}

\section{The ALCF is accelerating scientific discoveries in many disciplines, ranging from chemistry and engineering to physics and materials science.}

A research team from Argonne National Laboratory and the University of Chicago is using nanometerscale imagery of the enamel of a primate tooth to study its internal structure and test hypotheses related to mechanical characteristics. The data was imaged using high-resolution x-ray tomography at Argonne's Advanced Photon Source. To analyze the inherent structures of these large-scale experimental datasets, the team worked with ALCF researchers to visualize the data using the vl3 parallel volume rendering framework.

Image credit: James Grudzinski, Carmen Soriano Hoyuelos, Joseph A. Insley, and Silvio Rizzi, Argonne National Laboratory; Callum Ross,

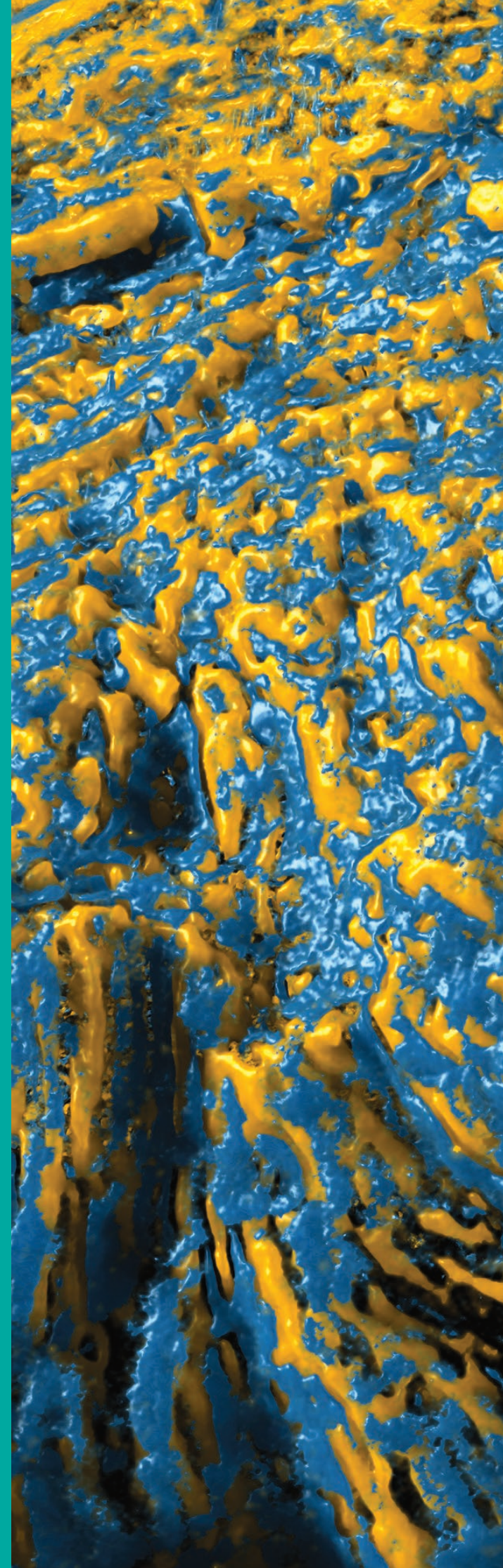




\section{c.}

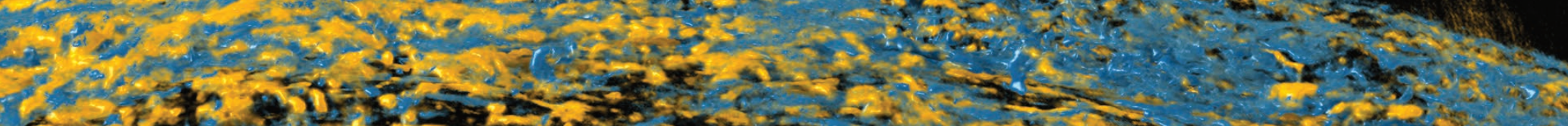

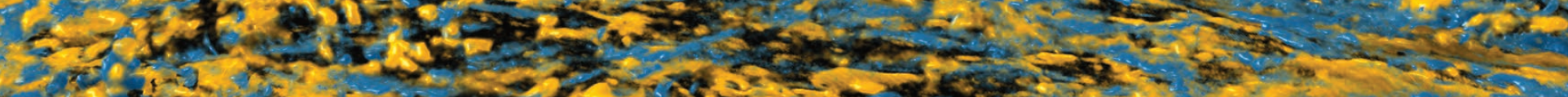

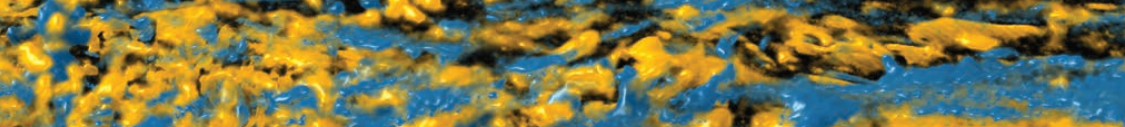

\section{are \\ 425}

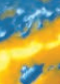

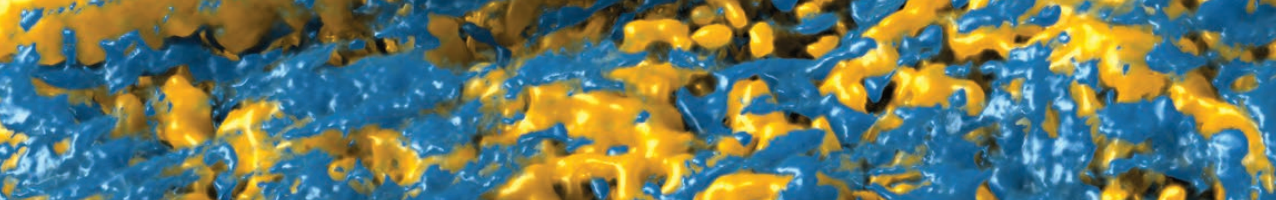

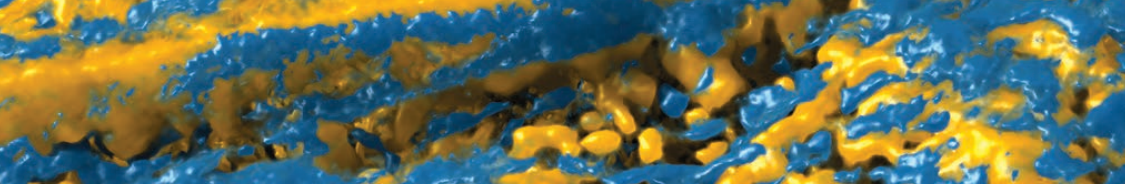

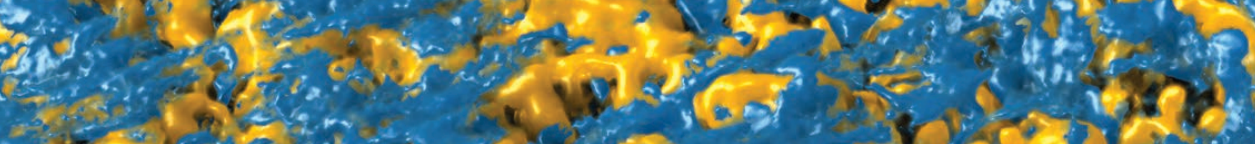

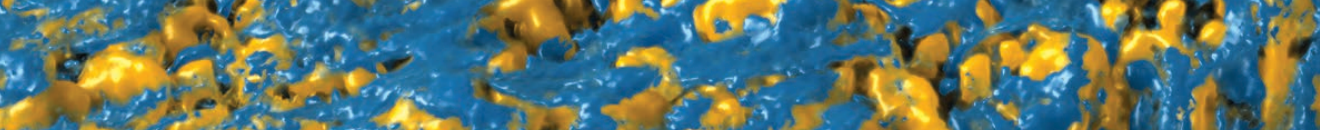
2. and sering

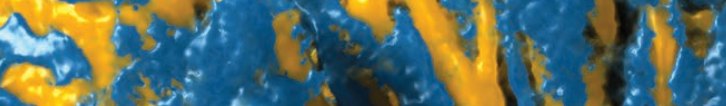
a - 7 Th st tive (1) 10.11:1. $\operatorname{lin}^{2}=$

ing

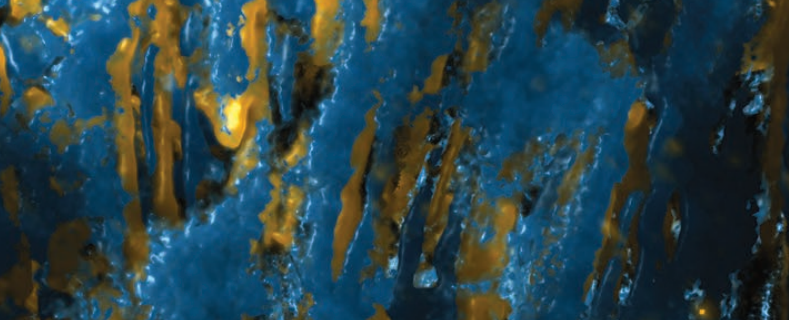

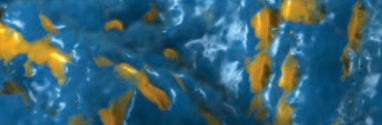

की

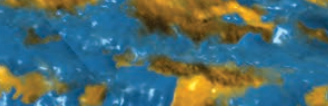

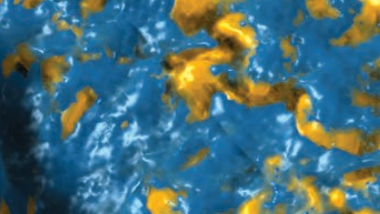
2y-2. 


\title{
ACCESSING ALCF RESOURCES
}

\begin{abstract}
As a national user facility dedicated to open science, any researcher in the world with a large-scale computing problem can apply for time on ALCF computing resources.
\end{abstract}

Researchers gain access to ALCF systems for

computational science and engineering projects, typically with awards of millions of core-hours, through competitive, peer-reviewed allocations programs supported by the DOE and Argonne.

The ALCF also hosts competitive, peer-reviewed application programs designed to prepare key scientific applications and innovative computational methods for the architecture and scale of ALCF supercomputers.

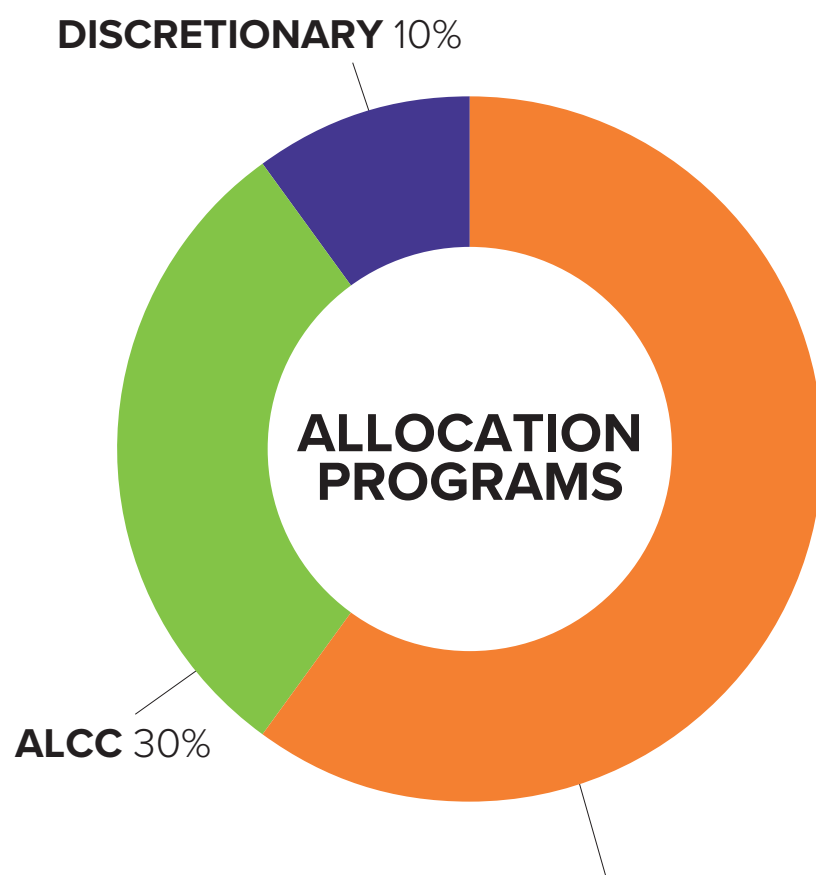

A breakdown of how computing INCITE 60\%

\section{APPLICATION PROGRAMS}

\section{Early Science Program (ESP)}

As part of the process of bringing a new supercomputer into production, the ALCF hosts the ESP to ensure its next-generation systems are ready for science on day one.

\section{ALCF Data Science Program (ADSP)}

Targeted at big data science problems, ADSP aims to explore and improve a variety of computational methods that will help enable data-driven discoveries across all scientific disciplines.

\section{ALLOCATION PROGRAMS}

\section{Innovative \& Novel Computational Impact on Theory and Experiment (INCITE)}

The DOE's INCITE program provides allocations to computationally intensive, large-scale research projects that aim to address "grand challenges" in science and engineering.

\section{ASCR Leadership Computing Challenge (ALCC)}

The DOE's ALCC program allocates resources to projects directly related to the DOE's energy mission, as well as national emergencies, and for broadening the community of researchers capable of using leadership computing resources.

\section{Director's Discretionary}

The ALCF's Director's Discretionary program provides "start-up" awards to researchers working toward an INCITE or ALCC allocation to help them achieve computational readiness. 

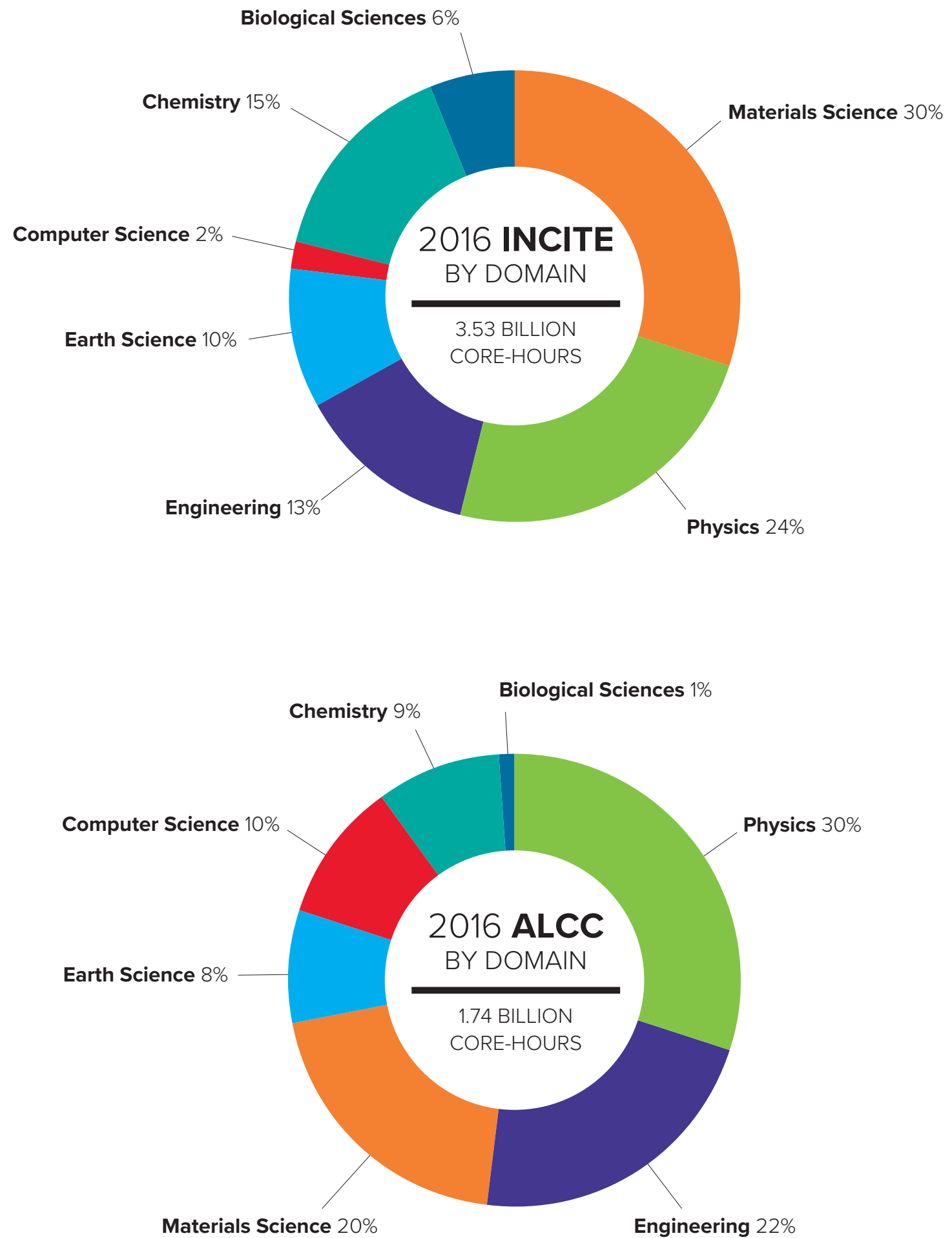

Note: ALCC data is from calendar year 2016 


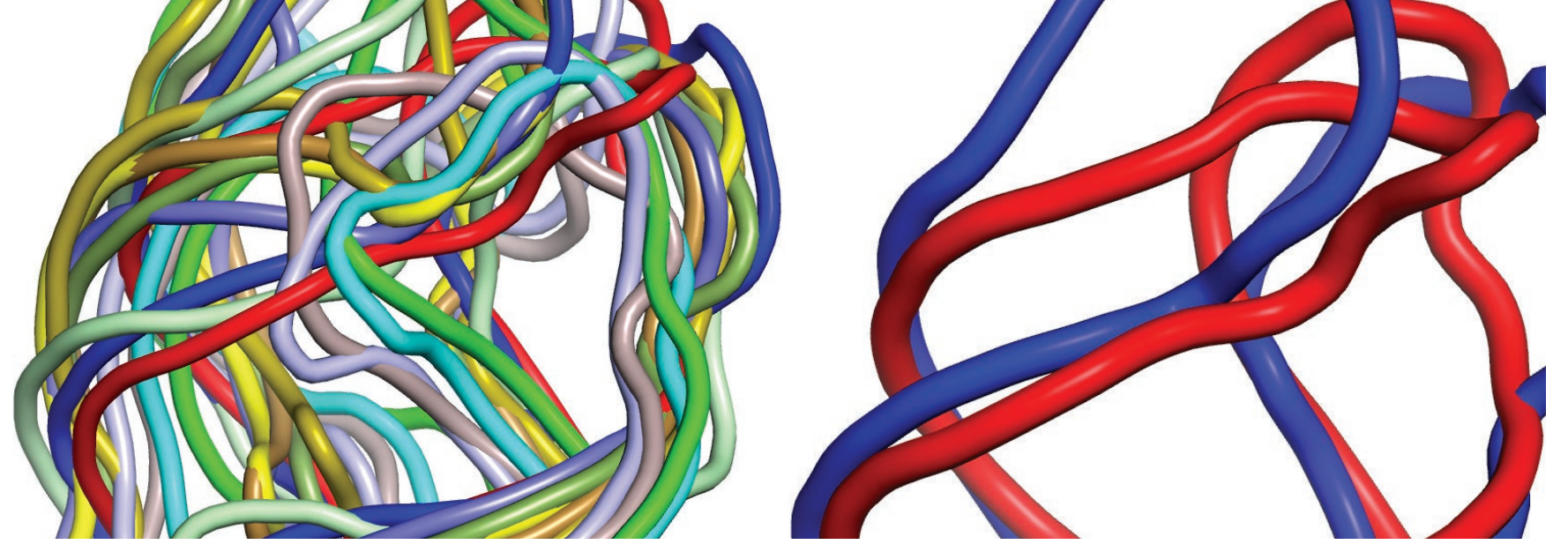

\section{COMPUTING 3D STRUCTURES OF RNA FROM SMALL-ANGLE X-RAY SCATTERING DATA AND SECONDARY STRUCTURES}

YUBA

BHANDARI

bhandariyr@mail.nih.gov

\section{YUN-XING \\ WANG}

wangyunx@mail.nih.gov

National Cancer Institute

\section{Director's \\ Discretionary}

15 Million Core-Hours

Biological Sciences
Image: Explicit 3D structures of adenine riboswitch RNA (PDB ID 1Y26) calculated using the RS3D program.

Image credit: Wei Jiang, Argonne National Laboratory; Yuba Bhandari and Yun-Xing Wang, National Cancer Institute
Ribonucleic acid (RNA) plays a critical role in regulating cellular processes, making it an important area of research for cancer studies. Gaining a better understanding of RNA depends on knowledge of its 3D structures, but such structures are difficult to ascertain with conventional methods. Researchers from the National Cancer Institute are using ALCF computing resources to develop a novel approach for calculating RNA structures that would greatly improve our understanding of RNA biology.

APPROACH The research team developed a robust algorithm and computational program called RS3D to calculate 3D structures of RNA using small-angle $x$-ray scattering (SAXS) data and known secondary structures as input. Starting from a glob model at nucleotide level of resolution, the algorithm carries out natural hierarchical moves based on the structural composition of RNA. Each move is guided toward improving the SAXS data fit and long-range interactions, if available. To conduct these types of calculations, the researchers must generate tens of thousands of structures for each type of RNA. Computing the RNA structures with various folds and complexities necessitates the use of a petascale supercomputer like Mira. In collaboration with ALCF researchers, the team has scaled and optimized RS3D to improve its performance on Mira.

RESULTS The team published a paper in the journal Methods detailing their computational approach to model RNA structures using SAXS data. They also validated the RS3D method extensively, using more than 15 different RNA structures that represent a wide variety of folding architectures available in the current structural database. By computing 3D topological RNA structures with diverse junction types and structural complexities, the team demonstrated the utility and robustness of RS3D, using simulated as well as experimental SAXS data. The method's novel features include conceptual simplicity that incorporates secondary structure motifs and the flexibility to integrate a variety of tertiary interactions that users can obtain from biochemical and biophysical data.

IMPACT This project is developing a high-throughput method that can determine the structure of RNA in a diverse folding landscape to fill in the gap between known RNA sequences and their explicit 3D structures. This work has significant implications in understanding the structural basis of RNA biology, and thus in the advancement of RNA therapeutics. 


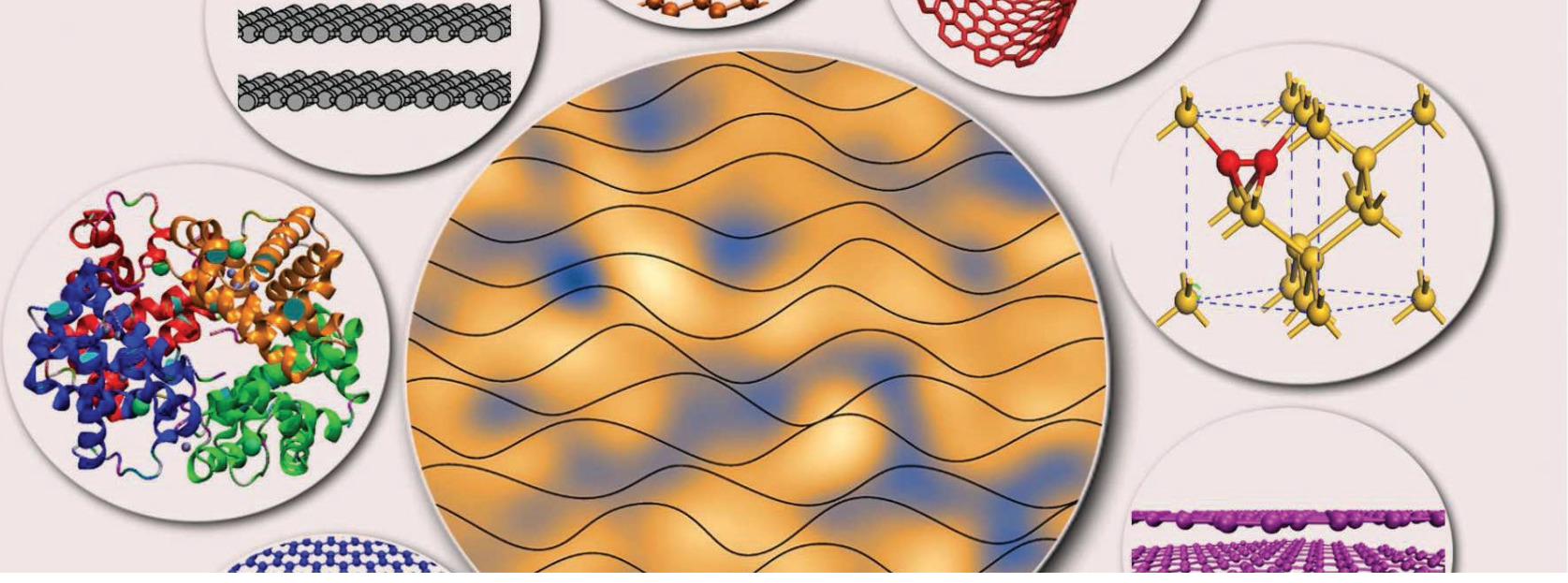

\section{ANOMALOUS DENSITY PROPERTIES AND ION SOLVATION IN LIQUID WATER: A PATH-INTEGRAL AB INITIO STUDY}

\section{ROBERT A. DISTASIO \\ distasio@cornell.edu Cornell University}

\section{ALCC}

175 Million Core-Hours Chemistry
Image: Researchers used Mira to validate a new many-body "wavelike" theoretical model to accurately solve for the van der Waals forces, demonstrating that this long-range interaction can be significantly enhanced at the nanoscale. This discovery is one component of the team's work, which involves carrying out large-scale simulations of liquid.

Image credit: Robert A. DiStasio, Cornell University; Alexandre

Tkatchenko, Fritz Haber Institute of the Max Planck Society and University of Luxembourg
Water is essential to life and critical to research fields addressing some of today's biggest energy challenges. Although the structure of a single water molecule is known, liquid water has a complex, disordered microscopic structure that is difficult to observe. At present, there is no experimental method that can probe the microscopic structure of liquid water, and many computerbased simulations, though useful for other types of microscopic molecular modeling, cannot reproduce the structure and dynamics of water and aqueous solutions important to applications, such as industrial catalysts, fuel cells, and protein stability.
APPROACH Researchers from Cornell and Princeton are performing highly accurate benchmark atomistic simulations of liquid water and aqueous ionic solutions through a combination of algorithmic advances and high-performance computing. The team has conducted large-scale simulations of liquid water, pyridine, and pyridine-like molecular crystals that are found in processes like DNA synthesis; and hydronium and hydroxide aqueous ionic solutions that provide fundamental models of acidic and basic conditions. Researchers are using the electronic structure code Quantum ESPRESSO (QE) to perform $a b$ initio molecular dynamics simulations utilizing density functional theory. ALCF staff helped improve the performance of QE simulations up to 40 percent by making better use of Mira's processors and reducing interprocessor communication.

RESULTS Results include a highly accurate characterization of microscopic structures and anomalous density properties of liquid water and crystalline ice; proton transfer rates and diffusivities of aqueous hydronium and hydroxide ions; and new insights into how the long-range van der Waals interactions and nuclear quantum effects affect the structure and equilibrium densities of pyridine and pyridine-like crystals. Related to the latter finding, the team recently validated a new "wave-like" theoretical model of the van der Waals force that better predicts how it behaves at the nanoscale. The results were published in the journal Science in 2016.

IMPACT For this project, researchers are simulating the microscopic structure and equilibrium properties of liquid water and aqueous ionic solutions with unprecedented accuracy, addressing important areas of renewable energy research, such as the design of aqueous ion batteries and fuel cells. 


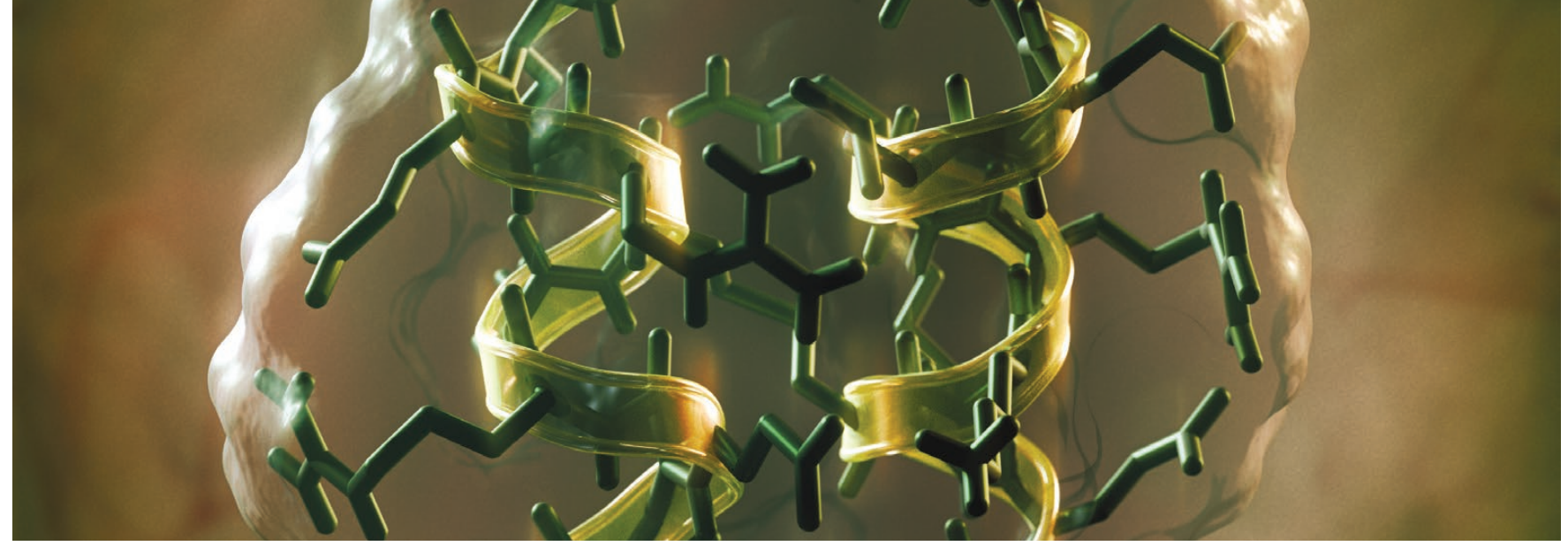

\section{TOWARDS BREAKTHROUGHS IN PROTEIN STRUCTURE CALCULATION AND DESIGN}

\section{DAVID \\ BAKER}

dabaker@u.washington.edu

University of Washington

\section{INCITE}

120 Million Core-Hours Chemistry
Image: This man-made peptide contains both natural and mirrorimage amino acid building blocks. This arrangement, which was modeled using Mira, gives rise to spirals that twist in opposite directions, a structure not seen in any natural proteins.

Image credit: Vikram Mulligan, University of Washington
Small-molecule drugs like aspirin use active ingredients that can cause unwanted side effects as they spread easily throughout the body. Protein drugs, designed to reduce side effects, can have the opposite problem - proteins can be too big, making it difficult to cross key membranes such as the blood-brain barrier. Researchers from the University of Washington are using Mira to develop and apply new computational methods for protein structure design, particularly that of small proteins called peptides, to further the impact of medicine.

APPROACH The Rosetta software suite, developed at the University of Washington's Baker Laboratory, is designed to tackle two difficult computational problems: the prediction of protein structure from amino acid sequences and the design of new amino acid sequences to yield a desired function. To enable the computational design work, the team developed a multistate design approach that allows them to create novel peptides with unique, rigid folds.

Their method involves exhaustively enumerating the possible conformations of peptides to search for a sequence that uniquely stabilizes one desired structure and destabilizes alternative structures. The search algorithm benefits enormously from Mira's massively parallel architecture, particularly when assigning each conformational state to a separate core for simultaneous sampling.

RESULTS The team made fundamental improvements to Rosetta's high-resolution energy function, including its agreement with observational studies and ability to reproduce and discriminate amino acid sequences. Using their multistate design approach, the researchers successfully designed stable versions of synthetic peptides. They validated the computational protocol by assembling physical peptides in the laboratory, which compared remarkably well with the simulated peptides. The team detailed their results in a paper published in Nature.

IMPACT This project is advancing protein structure modeling capabilities to enable the design of novel proteins, including therapeutic peptides that target diseases such as Ebola, HIV, and Alzheimer's. Artificial peptides represent a new class of drugs that have potential for greater efficacy and fewer side effects. The computational tools can also be used to design peptide catalysts and enzymes for environmental, energy, and industrial applications. 


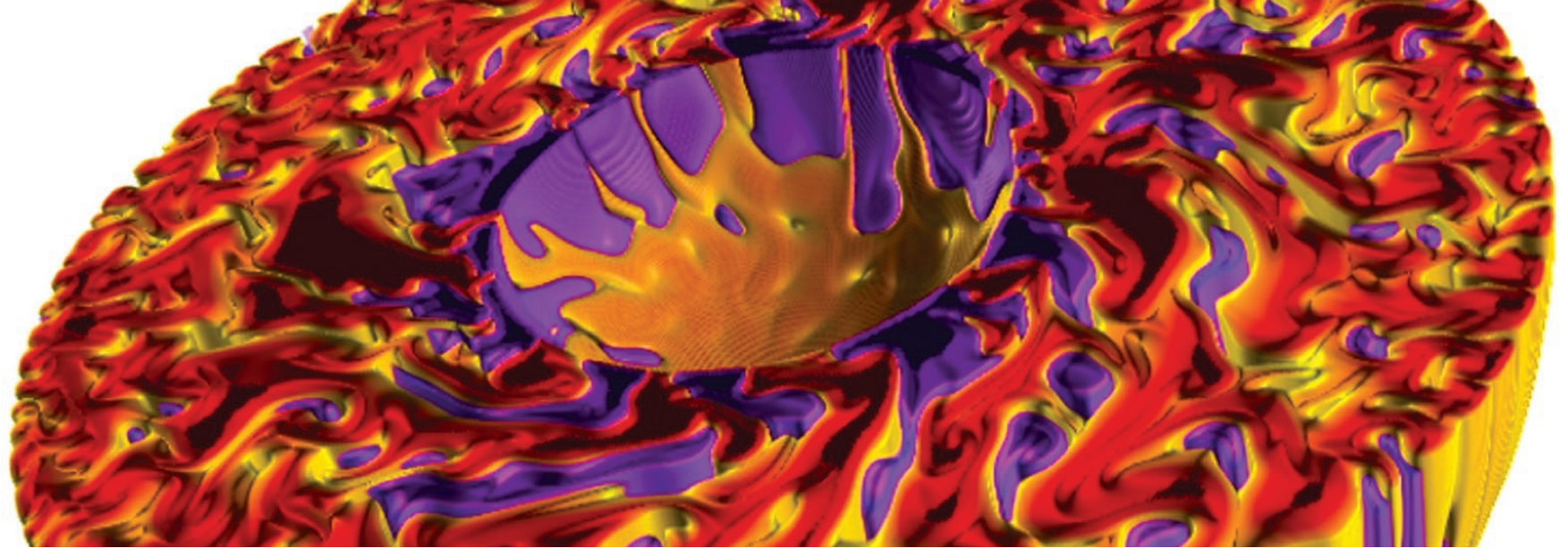

\section{FRONTIERS IN PLANETARY AND STELLAR MAGNETISM THROUGH HIGH-PERFORMANCE COMPUTING}

\section{JONATHAN AURNOU

\author{
aurnou@gmail.com \\ University of California, \\ Los Angeles
}

\section{INCITE}

83 Million Core-Hours Earth Science
Image: Temperature perturbations as realized in rotating, Earth-like convection at Ekman number 10-5. Warm temperature perturbations are rendered in yellow, cool in violet tones. The rendering has been cut at the equatorial plane to reveal the interior structure of convective columns that arise under such rotationally constrained conditions.

Image credit: Nicholas Featherstone, University of Colorado Boulder
Magnetic fields are generated deep within the interiors of stars and planets through dynamo action, where the motion of a conducting fluid produces an outward flux of heat. Solar and terrestrial magnetism, whether generated by iron in the Earth's core or dense plasma at the heart of the sun, plays an important role in our modern technological society, where Earth's magnetic field shields us from explosive, magnetically driven, solar phenomena. As the process of magnetic-field generation remains largely inaccessible to direct observation, researchers are leveraging ALCF computing resources to develop a new generation of models describing the dynamics at play in the Sun, Earth, and Jupiter.

APPROACH Through this ongoing investigation, a team of geo- and astrophysicists continue to develop state-of-the-art computational models to describe the interior dynamics of the Sun, Earth, and Jupiter. ALCF staff has participated in the application code development of Rayleigh, a pseudo-spectral code designed to study magnetohydrodynamic convection in spheres. Optimized to Mira, the code has allowed the team to construct high-resolution models detailing how convection transports energy generated by fusion deep within the Sun's core to the solar surface. This code and computer combination can resolve a range of spatial scales previously inaccessible to numerical simulation, ranging from the Sun's diameter to those roughly 1,000 times smaller.

RESULTS In 2016, the team added a new layer to the Rayleigh code, allowing it to solve on radially nested Chebyshev grids, which facilitates both higher accuracy and lower computational cost. While the team continues to evolve its solar simulations to include the effects of rotation on kinetic-energy scaling laws, simulations on Jovian dynamics have begun in earnest. They recently carried out the first survey to understand the appropriate internal heating functions for Jupiter simulations in preparation for primary, non-magnetic, Jupiter runs, which will constitute the highest-resolution Jupiter simulations yet.

IMPACT These models will open new windows into the understanding of the interplay of magnetism, rotation, and turbulent convection occurring within the remote interiors of geophysical and astrophysical bodies. The research will also provide the broader community access to the singular, extreme datasets generated by these massive computational efforts. 


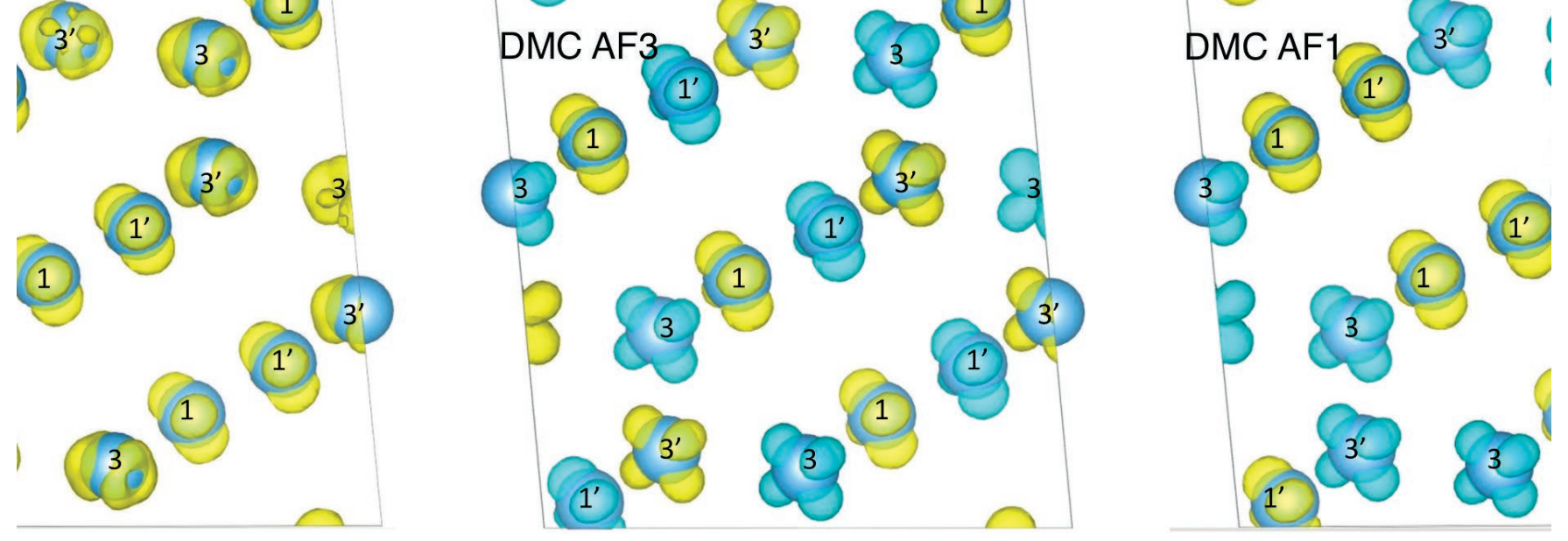

\section{QMC SIMULATIONS DATABASE FOR PREDICTIVE MODELING AND THEORY}

\author{
DAVID \\ CEPERLEY \\ ceperley@illinois.edu \\ University of Illinois at \\ Urbana-Champaign
}

\section{INCITE}

170 Million Core-Hours (ALCF: 90M; OLCF: 80M) Materials Science
Image: Diffusion Monte Carlo spin densities for low-temperature $\mathrm{Ti}_{4} \mathrm{O}_{7}$ ferromagnetic (left), antiferromagnetic 3 (center), and antiferromagnetic 1 phases. Yellow represents a positive spin density (or spin up) and blue

represents a negative spin density (or spin down).

Image credit: Anouar Benali and Olle Heinonen, Argonne National Laboratory

\section{Due to its numerical expense, quantum Monte Carlo (QMC) methods were once limited to model systems of small atoms or molecules. However, with supercomputers like Mira, QMC methods can be used for rigorous calculations on more complicated materials. This project is applying QMC on DOE leadership computing systems to improve predictive modeling of key properties found in energy-related or fundamental materials.}

APPROACH Researchers are using the QMCPACK code for a variety of studies, including heterogeneous catalysis of transition metal nanoparticles, magnetic phase transitions, properties of materials under pressure, and strongly correlated materials showing interesting electronic and magnetic properties. ALCF computational scientists have improved the performance of QMCPACK by developing an interface between the fragmented molecular orbital method and QMC to increase the model system size up to 10,000 electrons with minimum loss of accuracy. By rewriting the code in IBM-specific language for Mira's architecture, they have also improved code performance by 30 percent.

RESULTS For one study, researchers modeled $\mathrm{Ti}_{4} \mathrm{O}_{7}$, a titanium oxide that has a range of technological applications due to its complex electronic and magnetic structures. Simulations on Mira have, for the first time, calculated the lowest energy states of $\mathrm{Ti}_{4} \mathrm{O}_{7}$, revealing its magnetic properties and stable ground state. The team's results were published in Physical Chemistry Chemical Physics and will help improve the accuracy of other electronic structure modeling tools, such as the widely used density functional theory. Additionally, researchers are studying the intermediate concentrations of iron-bearing perovskite and post-perovskite phases of magnesium silicate to better understand the unusual properties observed in the core-mantle boundary layer. Further, they are simulating platinum (Pt) solids, nanoclusters, and surfaces to analyze the surface energies of Pt (111) and Pt (100), which are important in surface and catalytic applications.

IMPACT This project aims to advance the efficiency and global applicability of QMC through the development of tools that make systematic research less time intensive. Its results are providing accurate predictions for energy-related materials and processes, such as transition metal oxides useful for electronics applications, perovskite phases of magnesium silicate found in the Earth's mantle, and platinum surfaces utilized in catalytic applications. 


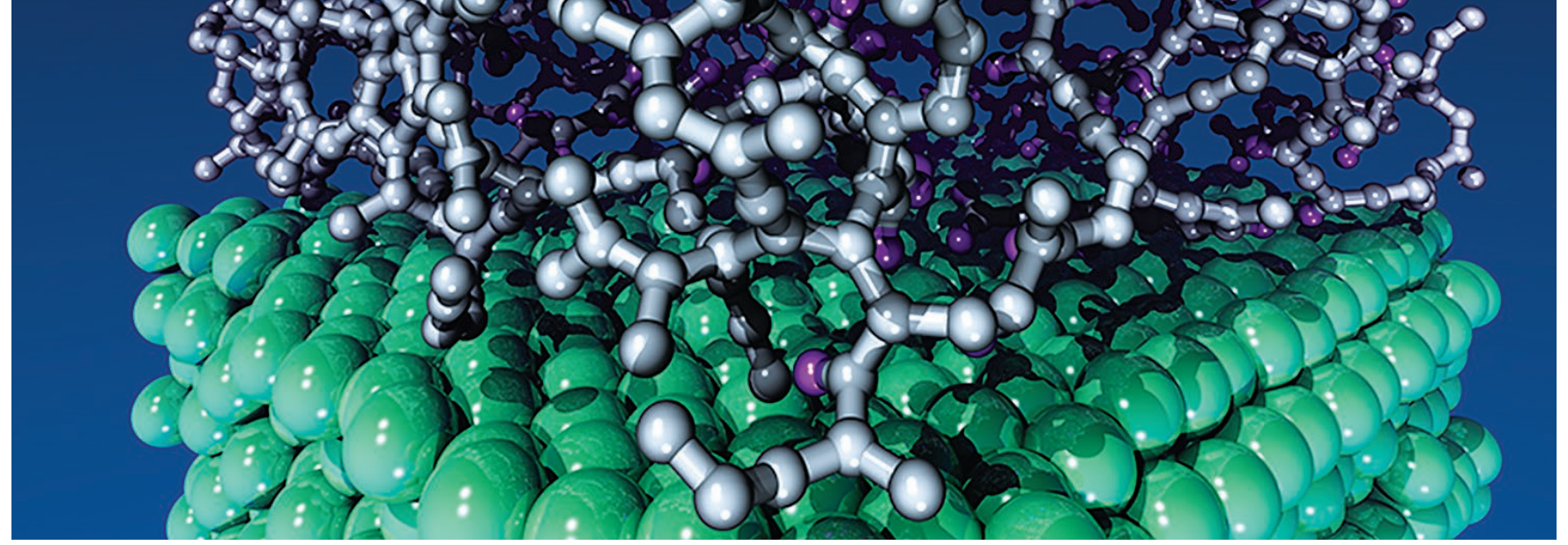

\section{REACTIVE MD SIMULATIONS OF ELECTROCHEMICAL OXIDE INTERFACES AT MESOSCALE}

\section{SUBRAMANIAN SANKARANARAYANAN \\ skrssank@anl.gov \\ Argonne National \\ Laboratory}

\section{INCITE}

40 Million Core-Hours Materials Science
Image: Visualization of a diamond-like carbon coating. Mira simulations have allowed an Argonne team to virtually test several potential catalysts (other metals and hydrocarbons in coatings and oils) for their self-healing properties in a high-temperature, high-pressure engine environment.
The initial reactions important to chemical bond formation, electron transfer, and other electrochemical processes can occur on the atomistic and molecular scale in a matter of nanoseconds, beyond the detection of many experiments. For the design and development of new materials for energy applications, scientists need a fundamental understanding of these underlying processes that facilitate desirable electrochemical reactions, including resistance to corrosion and friction. In this project, researchers are simulating the reactive processes, including bond breakage and formation, at electrochemical interfaces on the order of millions of atoms.

APPROACH Researchers from multiple computational and science domains at Argonne National Laboratory are simulating systems of over a million atoms on Mira using the LAMMPS molecular dynamics code with a ReaxFF module. ALCF staff assisted in doubling code performance by optimizing LAMMPS MPI communications on Mira's IBM Blue Gene/Q architecture and adding OpenMP threading to the ReaxFF module.

RESULTS After tribology experiments showed that a new film was being regenerated at the interface of an engine coating and base oil, researchers turned to Mira to model the underlying reactive processes. The simulations enabled the design of a self-healing, anti-wear coating that dramatically reduces friction and related degradation in engines and moving machinery. With LAMMPS, they modeled as many as two million atoms per simulation, making this one of the few atomistic studies of friction - of any kind, not just tribocatalysis - at this scale. Millions of time steps per simulation enabled researchers to identify the initial catalytic processes that occur within nanoseconds of machine operation. The results of the study were published in Nature. The researchers are now using their findings to virtually test other potential self-regenerating catalysts.

IMPACT Atomistic and molecular simulations are enabling researchers to understand the complex processes that make oils, coatings, electrodes, and other electrochemical interfaces effective. The computations are also allowing them to virtually test for new catalysts that may have useful properties. 


\section{PARTICLE ACCELERATION IN SHOCKS: FROM ASTROPHYSICS TO LABORATORY IN SILICO}

\author{
FREDERICO \\ FIUZA \\ fiuza@slac.stanford.edu \\ SLAC National \\ Accelerator Laboratory
}

\section{INCITE}

110 Million Core-Hours

Physics
Image: A visualization from a 3D OSIRIS simulation of particle acceleration in laser-driven magnetic reconnection. The trajectories of the most energetic electrons (colored by energy) are shown as (colored by energy) are shown as the two magnetized plasmas (grey isosurfaces) interact. Electrons are accelerated by the reconnection electric field at the interaction region and escape in a fan-like profile.

Image credit: Frederico Fiuza, SLAC National Accelerator Laboratory/ OSIRIS

\begin{abstract}
Magnetic reconnection, a fundamental process in astrophysical and fusion plasmas in which magnetic energy is converted into plasma energy, is thought to lead to efficient particle acceleration. The process is critical for the evolution of a variety of systems, from astrophysics to nuclear fusion devices, and holds promise as a means of explaining particle acceleration in explosive cosmic phenomena.
\end{abstract}

APPROACH Recent experiments studying laser-plasma interactions have shown the possibility of studying magnetic reconnection by using lasers to drive two plasma plumes that carry magnetic fields with opposite polarities. A laser blasts two foil targets, which are instantly turned into plasmas that expand and interact with each other. This INCITE project, an extension of similar work involving the development of particle acceleration in astrophysical shocks, is betting that these laboratory plasma experiments can shed light on the astrophysical processes that accelerate cosmic ray particles. Leveraging the relativistic massively parallel particle-in-cell (PIC) code OSIRIS, researchers conducted detailed simulations to study the possibility of observing particle acceleration in such an experimental setup - arguably the most important signature of reconnection. These were the first 3D simulations of laser-driven reconnection and the first to show the possibility of particle acceleration in this laboratory setting.

RESULTS The simulation results show that that laser-driven reconnection leads to strong non-thermal particle acceleration. As the two expanding plasma plumes interact with each other, they form a thin current sheet, or reconnection layer, which becomes unstable, breaking into smaller sheets. During this process, the magnetic field is annihilated and a strong electric field is excited in the reconnection region, strongly accelerating electrons as they enter the region. The accelerated electrons then escape in a fan-like profile, which leads to the development of a non-thermal particle distribution associated with some of the most explosive phenomena in the universe, such as solar flares and gamma ray bursts. The team's findings were published in Physical Review Letters.

IMPACT Results confirm that magnetic reconnection can lead to the efficient acceleration of astrophysical particles. The insight gained from these simulations may prove relevant not only to better understand the physics of cosmic rays, but also to design more efficient terrestrial accelerators for a variety of applications. 


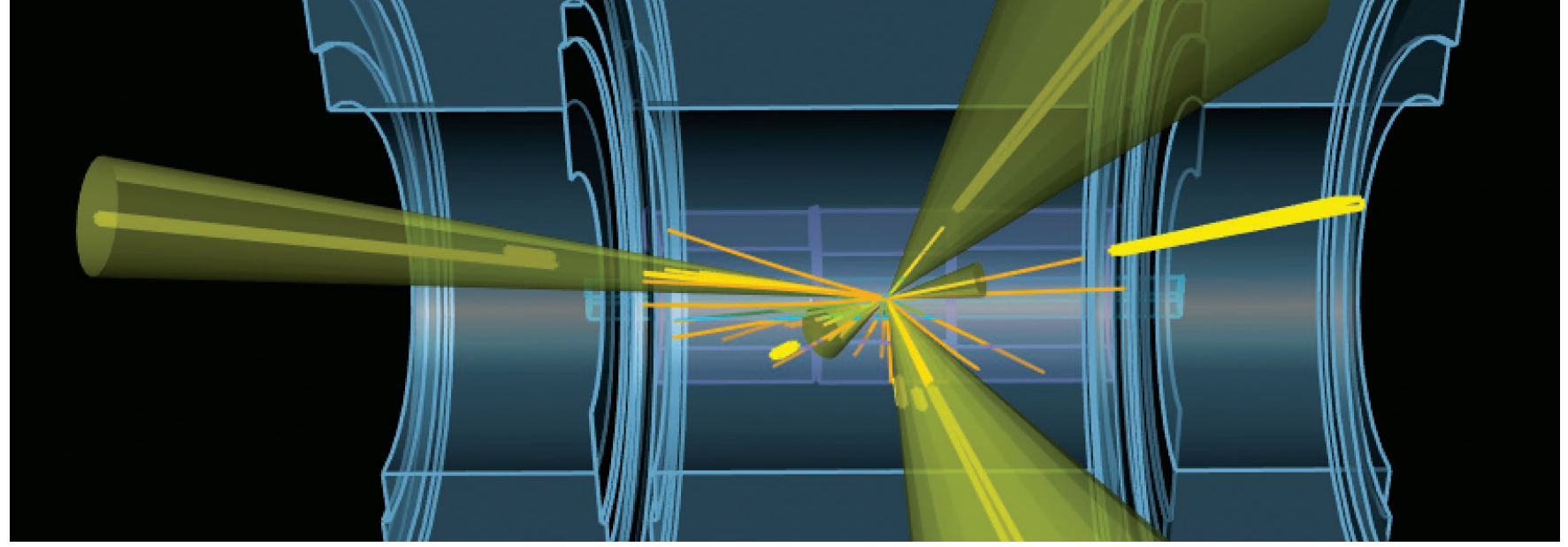

\section{PREDICTING THE TERASCALE ON-DEMAND WITH HIGH-PERFORMANCE COMPUTING}

\section{RADJA \\ BOUGHEZAL}

rboughezal@anl.gov

Argonne National

Laboratory

Director's

Discretionary

9 Million Core-Hours

Physics
With the theoretical framework

developed at Argonne, researchers can more precisely predict particle interactions such as this simulation of a vector boson plus jet event.
Scientists analyzing data produced during the high-energy particle collisions at the Large Hadron Collider (LHC) are looking for subtle deviations from the Standard Model of particle physics. Using precision calculations, researchers can resolve discrepancies between theoretical predictions and experimental data. In the first run of the LHC, scientists discovered the Higgs boson. Now operating at a higher collision energy in its second run, researchers anticipate that even more exact calculations of particle interactions may address other outstanding problems in fundamental physics, including the nature of dark matter and the origin of matter-antimatter asymmetry.

APPROACH In this large-scale computational study, researchers from Argonne National Laboratory developed a novel framework adapted for supercomputers to perform precision calculations that are critical to advancing knowledge of phenomena such as dark matter. They used two-thirds of Mira's more than 49,000 nodes to predict the process of vector boson plus jet production, a particle interaction that is the dominant background in dark matter searches and has been measured by LHC experiments. The team's new framework maps the high-energy physics calculations to three separate many-dimensional integrals that are performed using importance-sampling Monte Carlo integration parallelized via MPI+OpenMP. Because this approach uses many sampling points to evaluate these integrals over the possible particle trajectories, Mira's massively parallel architecture is needed for unprecedented accuracy.

RESULTS The theoretical predictions produced on Mira explain an important class of measurements that had previously resisted theoretical explanation and will better enable researchers to track the rate of dark matter events in contrast to the barrage of similar events that take place in collisions. Results were published in a 2016 issue of Physical Review Letters.

IMPACT This project demonstrates that supercomputers can improve our ability to predict fundamental particle interactions in nature at the highest energies, making the most of experimental data and expediting searches for unknown particles and interactions. 


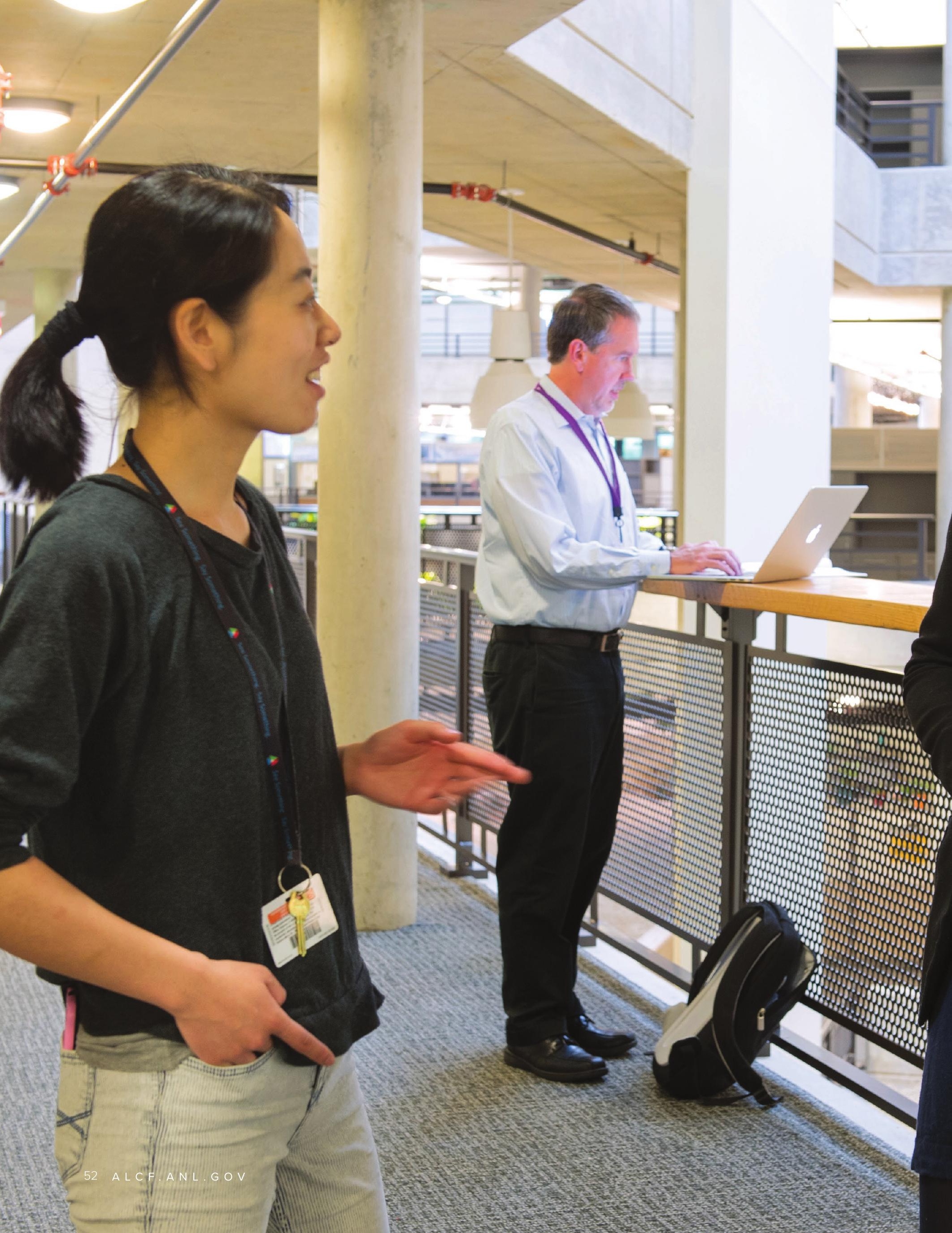




\section{EXPERTISE \& RESOURCES}

The ALCF's unique combination of supercomputing resources and expertise enables breakthroughs in science and engineering that would otherwise be impossible. 


\section{STATS}

\section{OPERATIONS}

The Operations team is responsible for managing and supporting all ALCF computing systems, ensuring users have a stable, secure, and highly available resource to pursue their scientific goals. This includes overseeing the facility's supercomputers and computing clusters, network infrastructure, storage resources, and systems environments. In addition, the team develops and maintains a variety of tools that are critical to the seamless operation of the ALCF's supercomputing environment.

\section{SCIENCE}

The Science team works directly with ALCF users to maximize and accelerate their research efforts using the facility's computing resources. With multidisciplinary domain expertise, a deep knowledge of the ALCF computing environment, and experience with programming methods and community codes, the Science team helps users improve scientific productivity on ALCF computing resources and surmount any obstacles they may encounter along the way. The team is comprised of computational scientists, performance engineers, visualization experts, and data science experts.

\section{USER EXPERIENCE}

The User Experience team supports the ALCF user community by providing technical assistance, managing outreach and training, and stewarding stories about activities and research conducted at the facility. User Experience staff also lead the ALCF's efforts in industry outreach and business intelligence. The team's wide-ranging efforts include managing various user processes and interfaces, operating the help desk, facilitating user training events, developing facility stories, growing the base of industry users, and assimilating and verifying data to ensure accurate reporting of facility information.

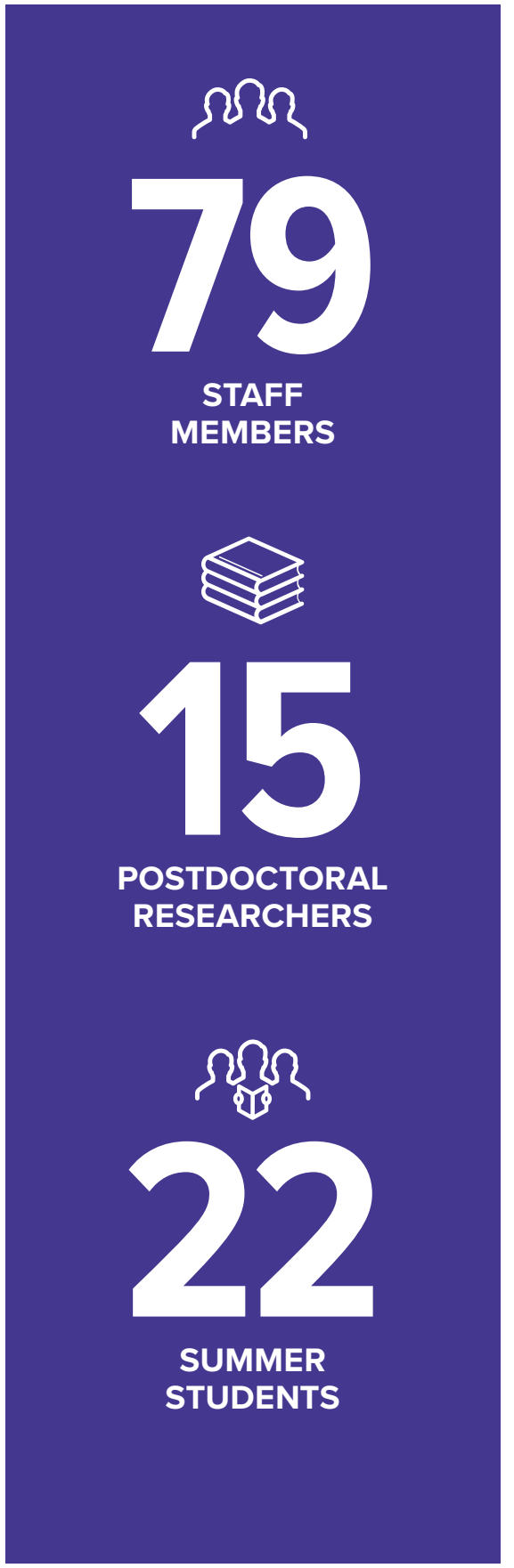




\section{STAFF NEWS}

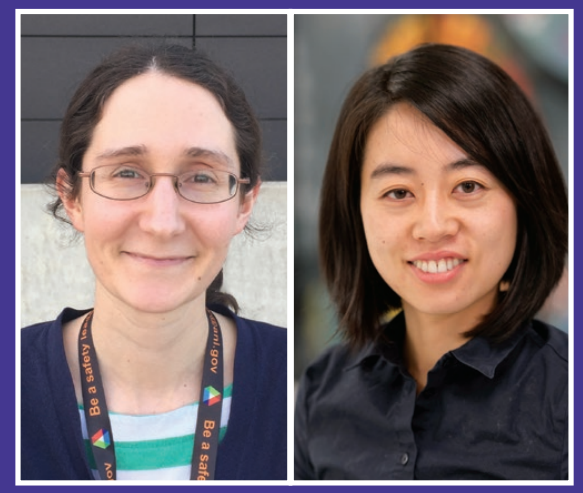

\section{ALCF}

\section{Announces its Next Margaret Butler Fellow}

In June, the ALCF named lowa State University graduate Colleen Bertoni as the next recipient of its Margaret Butler Fellowship in Computational Science. Bertoni, who was introduced at Argonne's annual Margaret Butler Celebration, will join the ALCF in 2017 to advance quantum chemistry studies of liquid water and ion solvation by employing and optimizing ab initiobased fragmentation methods on the facility's supercomputers.

Bertoni will replace Amy Ying Li, the ALCF's first-ever Margaret Butler Fellow. A postdoctoral researcher from the University of Southern California, Li's two-year fellowship was focused on using ALCF supercomputers to study materials for advanced batteries. She is now continuing her work at the laboratory as an Argonne Research Scholar.

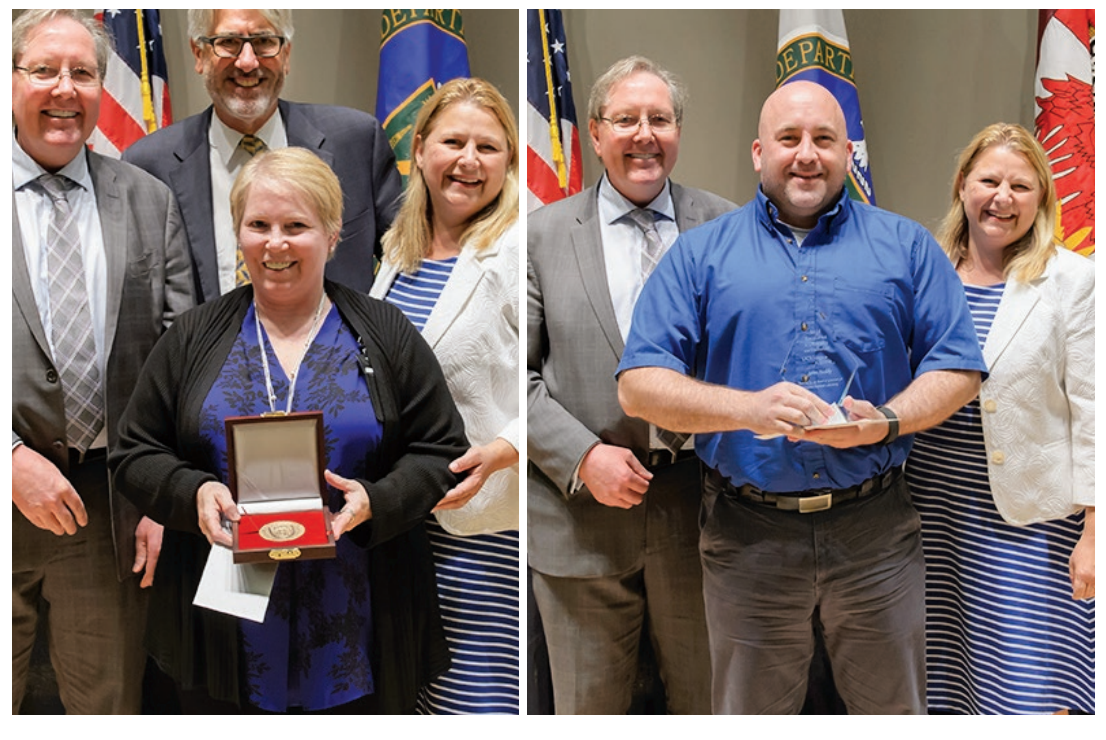

\section{Two ALCF Staff Members Recognized by Argonne's Board of Governors}

\section{Susan Coghlan and Skip Reddy}

of the ALCF were recognized for their outstanding achievements to the laboratory at the 2016 University of Chicago, Argonne, LLC Board of Governors' awards ceremony in August.

Coghlan, who has served as the project director for all ALCF supercomputers, received the Distinguished Performance Award for her exceptional accomplishments in petascale computing technologies, advanced system software, and large-scale computational facilities - an area that Argonne has defined as one of its strategic initiatives and that the DOE has identified as critical to solving the nation's most important scientific and engineering problems.

Reddy, the ALCF's HPC Infrastructure Lead, was awarded the Excellence in Diversity and Inclusion Award for his founding and continued stewarding of Spectrum, an employee resource group for the lesbian, gay, bisexual, transgender, queer, and ally community at Argonne. As a result, he has helped promote a more welcoming and inclusive laboratory community. 


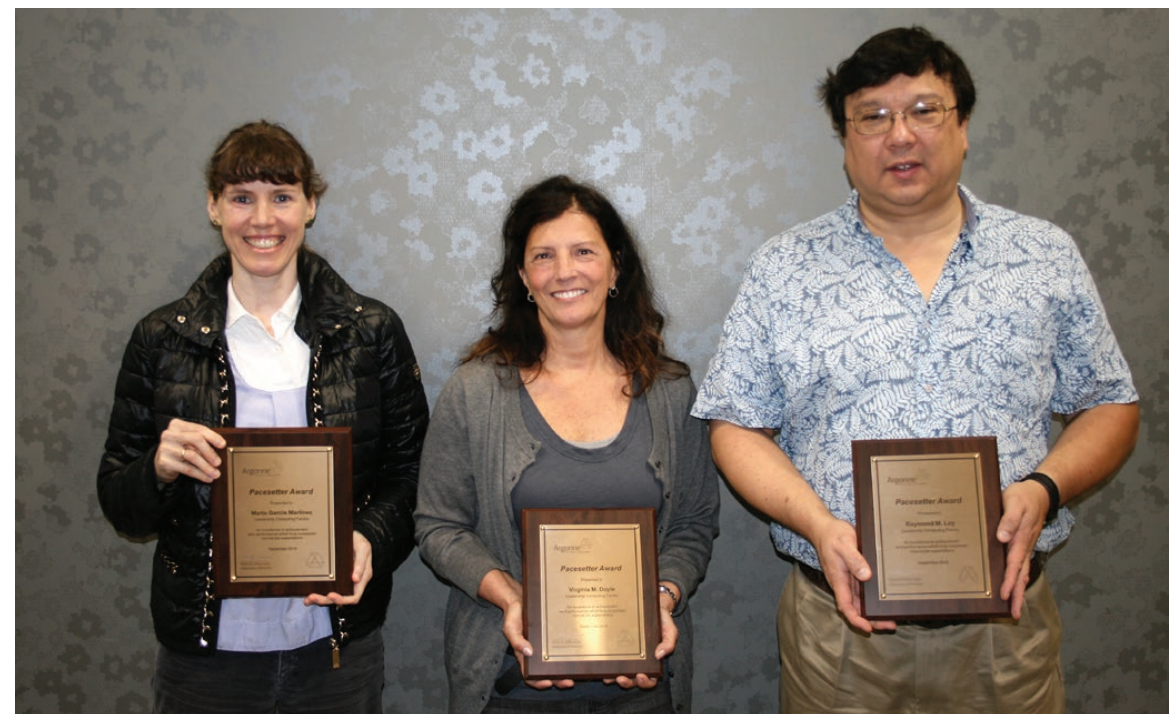

\section{Several Staff Members Honored with Pacesetter Awards}

\section{In 2016, 13 ALCF staff members received Argonne Pacesetter Awards, which are given to employees who have shown extraordinary initiative and dedication in their work at the laboratory.}

Ben Allen was recognized for his role in making the Joint Laboratory for System Evaluation a critical resource for the laboratory, and for his efforts in support of the Theta Early Science Program.

\section{Paul Coffman and William Scullin} were recognized for their effort to bring the Joint Center for Energy Storage Research's Materials Project to production state on Mira.

Ginny Doyle was recognized for her contributions to the 2016 Argonne
Training Program on Extreme-Scale Computing, including her support of the event website and other communications-related activities.

Marta Garcia and Ray Loy were recognized for their leadership in organizing and directing the 2016 Argonne Training Program on Extreme-Scale Computing.

Ye Luo was recognized for his work in implementing innovative technical improvements to advance the capabilities of the QMCPACK code.

\section{Kevin Harms, Ed Holohan, Ryan} Milner, Eric Pershey, Skip Reddy, and Paul Rich were recognized for their efforts and expertise leading up to and during the installation and acceptance of Theta.

\section{ALCF Fills Two Leadership Positions}

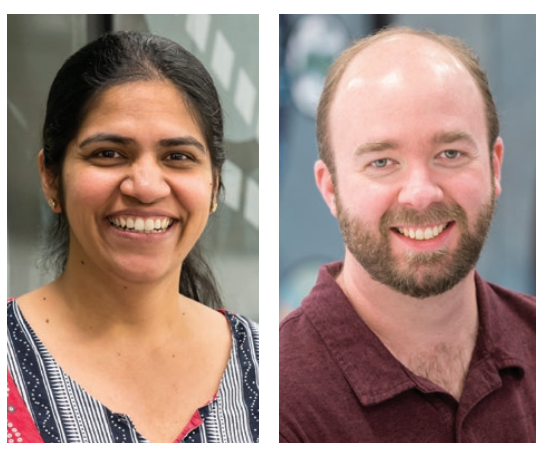

The ALCF filled two key leadership positions in 2016, with Jini

Ramprakash hired as deputy division director and Ti Leggett coming aboard as the deputy project director/deputy director of operations.

In her new role, Ramprakash, who previously served as ALCF's user experience team lead, works closely with the ALCF director in planning, developing, implementing, and evaluating major initiatives of the division, including day-to-day operations and facility development. Leggett comes to the ALCF from Argonne's Computing, Environment, and Life Sciences Directorate where he served as HPC systems administrator lead. In his role as deputy project director, Leggett assists the ALCF's project director in leading and managing large system deployment efforts, including Theta and Aurora. As deputy director of operations, he helps coordinate and manage the facility's operational activities. 


\section{STAFF SPOTLIGHTS}

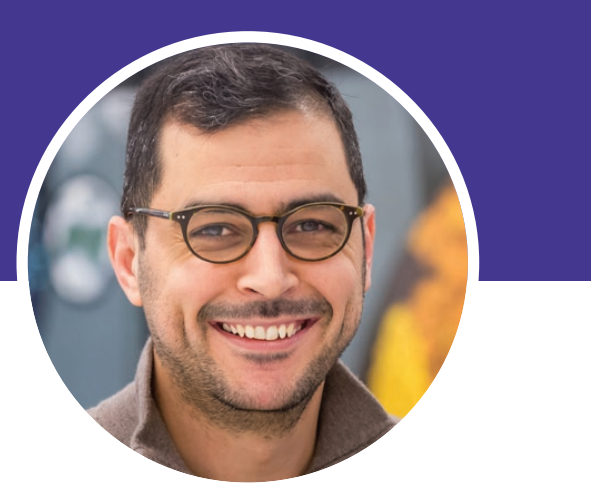

\section{Anouar Benali Computational Scientist}

Anouar is one of the HPC community's foremost experts on the quantum Monte Carlo (QMC) approach, an important computational tool for performing accurate electronic structure calculations of atoms, molecules, and solids. As a developer of the QMCPACK simulation package, his work focuses on implementing and improving QMC algorithms for current supercomputers and future exascale systems. In 2016, Anouar began serving as co-principal investigator of a new Intel Parallel Computing Center, a collaborative effort aimed at optimizing QMCPACK for massively parallel computer systems based on the Intel ${ }^{\circledR}$ Many Integrated Core Architecture technology. He was also named co-principal investigator of an application development project involving QMPACK for DOE's new Exascale Computing Project. In addition, Anouar's recent work with an INCITE research team demonstrated the ability to use QMC simulations to accurately calculate the magnetic properties of a titanium oxide material for the first time. Their results were published in Physical Chemistry Chemical Physics.

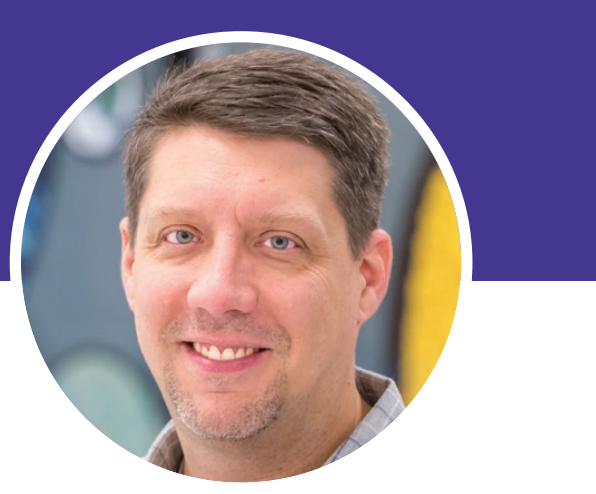

\section{Kevin Harms Performance Engineer}

From playing a lead role in the effort to deliver the facility's next-generation systems to working with users to optimize code performance, Kevin contributes to the ALCF mission in a multitude of ways. In 2016, he was a part of the team responsible for the successful acceptance of Theta. As the co-lead for the Aurora NRE (non-recurring engineering) effort, Kevin continues to work directly with Intel and Cray to resolve technical issues and to ensure all milestones are achieved. In the computational science realm, Kevin recently leveraged his I/O expertise to help an engine research team greatly improve the performance of the CONVERGE code on Mira. His optimization work enabled the team to carry out the largest engine simulation of its kind. Kevin is also a regular collaborator with the $1 / O$ research team in Argonne's Mathematics and Computer Science Division. Their work has included developing and deploying Darshan, a lightweight I/O instrumentation library, on ALCF systems to investigate the $1 / 0$ behavior of production applications.

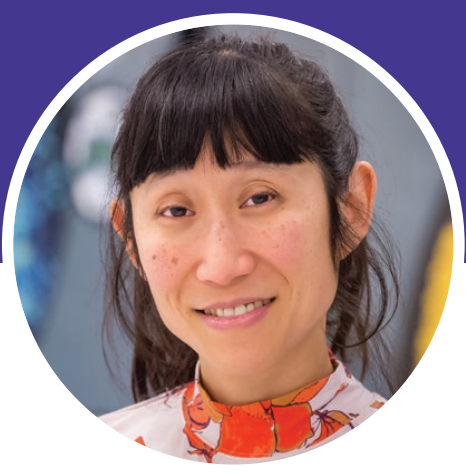

\section{Janet Knowles Software Developer}

Prior to joining the ALCF's visualization team, Janet spent nearly a decade in the research and development industry working with mixed reality environments, 3D modeling, and real-time computer graphics. She is now leveraging that expertise to develop new ways for ALCF staff and users to interact with their projects. As her first order of business, Janet was charged with creating ALCF on the Move (OTM), a mobile productivity app that allows staff and users to access real-time information on ALCF resources anywhere at anytime. From collecting requirements and designing the user interface to writing the software front- and back-ends, Janet handled all aspects of developing ALCF OTM. Currently available to staff, the app provides information on ALCF system availability, running and scheduled jobs, and ALCF news and events. Janet is now preparing ALCF OTM for wider release so facility users can take advantage of the new tool as well. Also active in mentoring at the ALCF, Janet has worked with several undergraduate and graduate students on in-house visualization projects and libraries. 


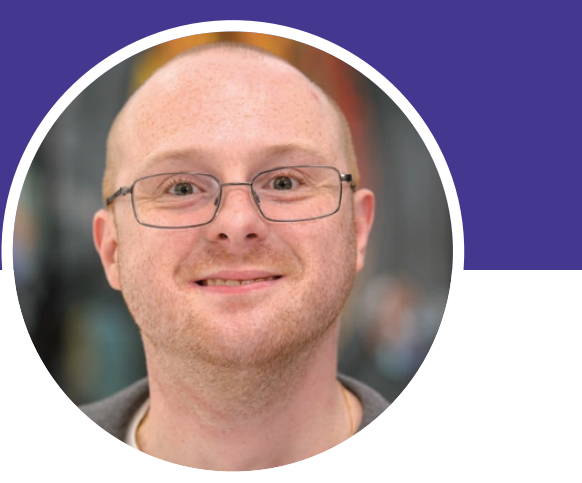

\section{Ben Lenard Database Administrator}

Ben is responsible for overseeing the administration and improvement of database systems in the ALCF's supercomputing environment. These databases are critical to many of the facility's support services, including job scheduling, job accounting, and business intelligence. In 2016, Ben deployed the IBM Data Server Manager to help streamline database administration tasks. With this tool in place, Ben has a better idea of how the databases are being used, while developers have an improved method for identifying and addressing any performance issues with their queries. Ben gave a presentation on his experience with the tool at the 2016 IBM World of Watson conference in Las Vegas. In addition to his day-to-day responsibilities, Ben has been a strong advocate for the ALCF and for computer science, volunteering for events like the Hour of Code and Argonne's public open house. He is also currently pursuing a PhD in Computer and Information Sciences at DePaul University.

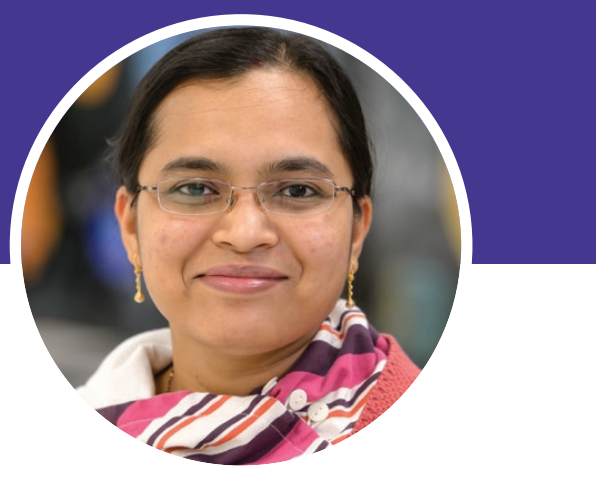

\section{Preeti Malakar Postdoctoral Researcher}

For her doctoral studies at the Indian Institute of Science, Bangalore, Preeti worked on efficient high-fidelity weather simulations, online data analysis, and remote visualization in resource-constrained computational environments. As a postdoctoral researcher with the ALCF's visualization team, she is now building off of her previous work to develop novel computational methods for some of the world's most powerful computing resources. Preeti's work includes developing in situ analysis techniques for large-scale molecular dynamics and astrophysics simulations, devising solutions to execute optimal

simulation-analysis workflows, and improving I/O performance through better routing of data flows. In 2016 , she co-authored a paper in the journal Parallel Computing that details a new algorithm designed to improve $\mathrm{I} / \mathrm{O}$ performance on Mira. By routing data to the closest $\mathrm{I} / \mathrm{O}$ nodes and reducing network congestion, the algorithm successfully reduced write times by an average of 60 percent over the default independent I/O.

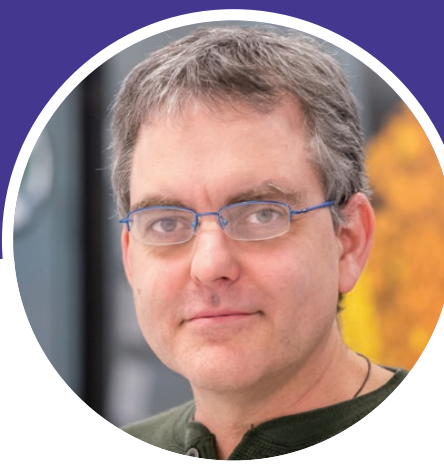

\section{Doug Waldron Data Architect}

In a Star Trek Borg-themed talk to ALCF staff earlier this year, Doug explained that the ultimate goal of his business intelligence team is "achieving data perfection." Since joining the ALCF in 2013, Doug has become the facility's go-to guy for all data-related inquires, which can range from machine utilization graphs to information about the ALCF user community. As an example, Doug's team is responsible for providing data for the ALCF's annual Operational Assessment Report, which calls for 63 metrics, 23 graphs, and 12 reports. Under his leadership, the small, but productive business intelligence team has developed automated tools to assimilate and perfect erratic and conflicted data from several different systems at the facility. In 2016, Doug led the expansion of the ALCF's Data Warehouse to include data from Theta, Darshan, Tracklib, and Autoperf. All in all, his business intelligence efforts have helped to eliminate the burden of data collection, maintenance, analysis, and reporting from several other groups at the ALCF. 


\section{ALCF SOFTWARE RESOURCES}

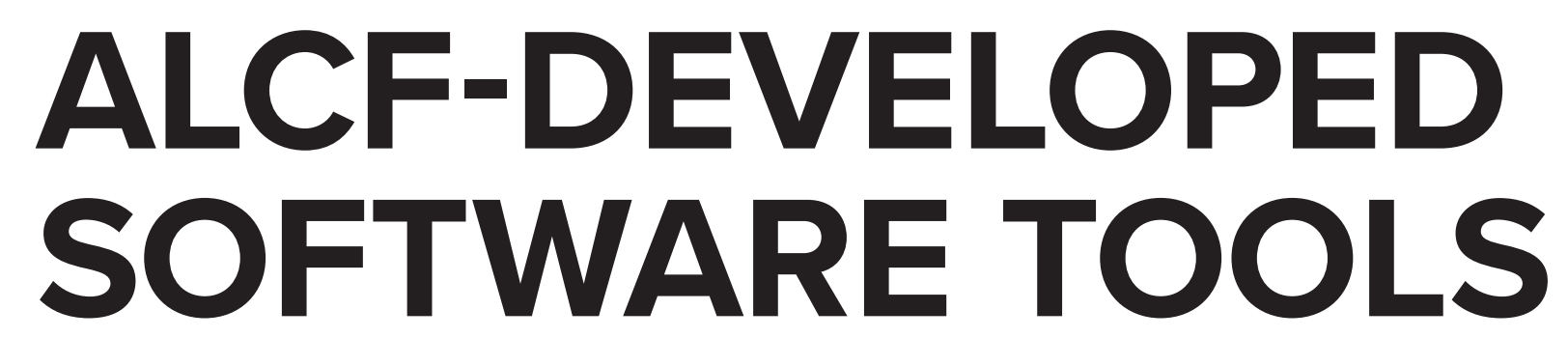

\section{ALCF researchers are developing software tools and approaches to help scientific applications run more efficiently on its leadership-class systems, and to provide valuable information to guide future support and research priorities.}

\begin{abstract}
AutoPerf
AutoPerf is an ALCF-developed library that automatically collects performance information for applications running on ALCF systems. The tool records data from simulations and saves it for analysis when the job is completed. Output includes MPI usage and performance information that indicates which MPI routines were called, how many times each routine was called, the time spent in each routine, the number of bytes sent or received (if applicable), and data from the system's hardware performance counters. This information is helping ALCF staff better understand the requirements of applications and how to optimize their performance.
\end{abstract}

\section{Business Intelligence Tools}

The ALCF's Business Intelligence (BI) team is responsible for assimilating and verifying data from many ALCF systems to ensure the accurate reporting of facility information to users, ALCF leadership, and DOE. This includes producing everything from real-time usage graphs to facility data for quarterly and yearly reports. The BI team has developed automated systems to continuously extract the data from several disjointed systems at the facility and convert the data into dimension and fact tables with a standard set of units. The data is loaded into a centralized database known as the Data Warehouse. Additional processing combines multiple datasets into smaller datasets suitable for use by the BI team's reporting software. The team has automated tools in place to ensure the accuracy and completeness of all data pulled from the Data Warehouse. $\mathrm{Bl}$ also maintains Pentaho, an ad hoc reporting system that allows ALCF staff members to execute complex queries and visualize resulting datasets. These efforts have greatly improved the efficiency of data collection, maintenance, analysis, and reporting at the ALCF.

\section{Cobalt}

Developed at Argonne, Cobalt is the ALCF's job scheduler and resource manager. This tool gives the facility great flexibility in executing its scheduling policies, accommodates diverse workloads, and provides important features (e.g., alternate kernel support) that are either not supported or poorly supported by other schedulers and resource managers. In 2016, the ALCF continued work to prepare Cobalt for its next-generation systems. The tool now has the ability to interface with and run on Cray systems, starting with the XC40 platform, significantly broadening the types of systems on which Cobalt can be installed. This functionality enabled ALCF researchers to use Cobalt to drive the successful acceptance testing of Theta. In addition, Cobalt's data-staging capabilities were enhanced to allow users to stage specific files to ALCF storage from external facilities, such as Argonne's Advanced Photon Source, using Globus Online to broker the transfer in a secure manner. This Cobalt improvement is also a critical first step to adding more data-centric features, such as handling non-volatile node-local storage and burst-buffer capabilities, in preparation for Aurora. 


\section{Compiler and Library Tracking}

The ALCF has developed and deployed two software tools Trackdeps and Tracklib - to track the compilers and libraries being used on its systems. The first stage in the library-tracking process is Trackdeps, which records paths to all build process inputs that contribute to the final output, including compiler identity, header files, Fortran module files, and libraries. Tracklib, the second stage in the library-tracking process, is a set of tools used to examine programs as they run on Mira, producing the data necessary to match the job's accounting information with the data collected by Trackdeps. With these tools in place, staff can determine how various libraries and compilers are being used at the facility, providing knowledge that helps inform support and ALCF and DOE research priorities.

\section{Darshan}

ALCF researchers are collaborating with staff from Argonne's Mathematics and Computer Science Division on the continued development of Darshan, a scalable HPC I/O instrumentation library designed to capture an accurate picture of application I/O behavior. This tool records statistics such as the number of files opened, time spent performing $1 / O$, and the amount of data accessed by an application. Darshan's lightweight design makes it suitable for fulltime deployment for workload characterization of large systems. The information revealed by Darshan enables researchers to investigate and tune the $1 / O$ behavior of complex HPC applications, while also helping the storage research community to

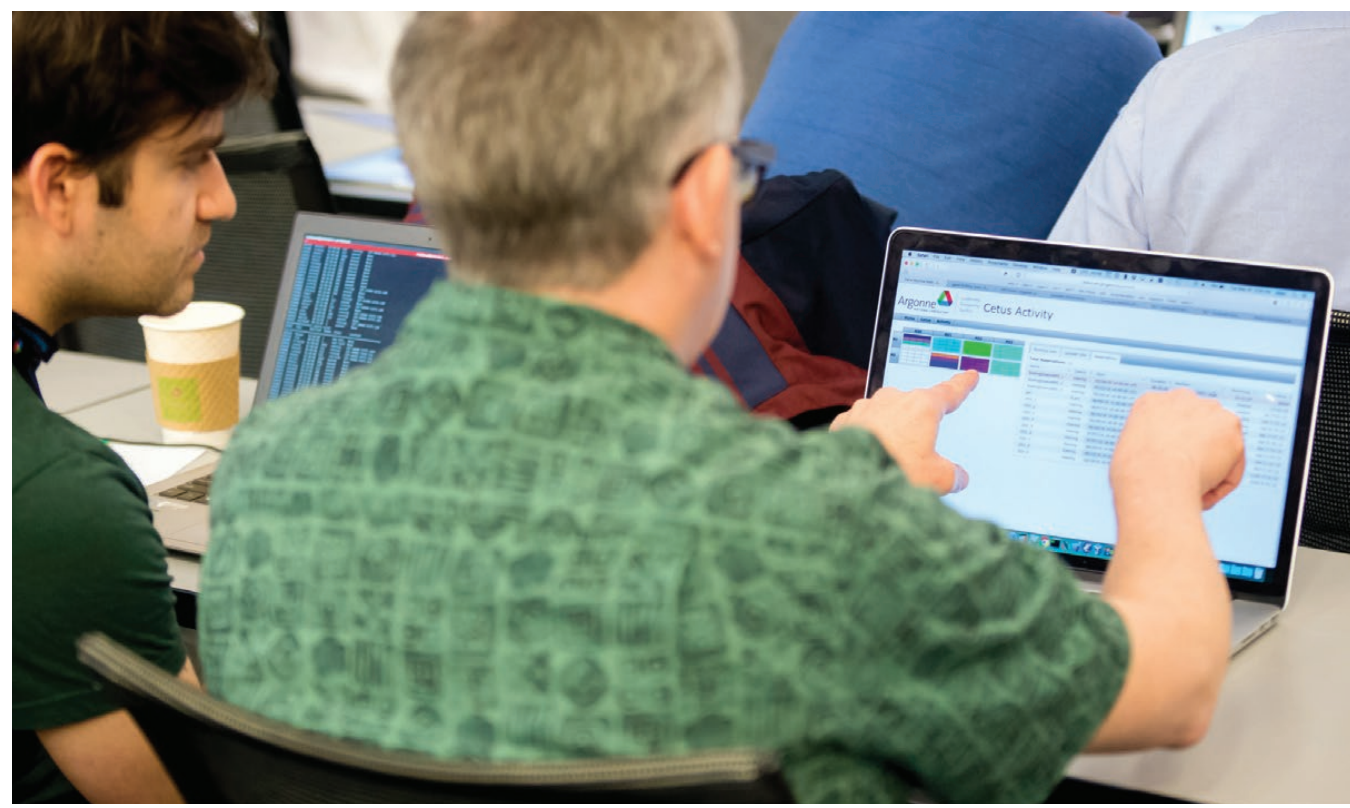

A web-based tool called the Gronkulator pulls machine status information from Colbalt to provide users and staff with a real-time look at ALCF system activity.

develop strategies and approaches to better serve the needs of scientific computing. Darshan is portable across a wide variety of platforms and is now deployed at multiple DOE computing facilities. In 2016, the development team released a new modularized version of Darshan (version 3).

\section{Job Failure Analysis}

Every week, the ALCF Operations team analyzes all job failures that occur at the facility to resolve any system-related issues and to identify issues that could help improve user productivity. To help with this analysis, the ALCF has developed a system to allow interactive querying of job data and annotation of job status. This system helps ALCF staff determine the fate of every job with a non-zero exit code. Instead of many people talking to the one person who has specific job information, the system allows staff to save the data in a database in real time. The system has also eliminated much of the manual log parsing that individuals were doing to find out what really happened to a particular job. In 2016, ALCF staff began adapting the job failure analysis software for its next-generation systems, Theta and Aurora, as part of the development of the Operational Data Processing Service (ODPS). In addition to being the core of the job failure analysis data, ODPS tracks usage, availability, reservations, jobs, or any event that happens over time using an ALCF computing resource.

\section{sbank}

In December, the ALCF deployed sbank, a new job accounting system that will replace the facility's original accounting tool, cbank. The customized software system, which processes output from the Cobalt scheduler, is designed to allow users to track project allocations, usage charges, and refunds. It gives users the ability to query the balance and expiration of their project, and enables them to manage and monitor their allocation usage by user, job, and computing system. The new sbank software has similar syntax to cbank, but provides enhanced options for displaying allocation information, giving users much more flexibility in how they can query and report usage data. 


\section{ALCF COMPUTING RESOURCES}

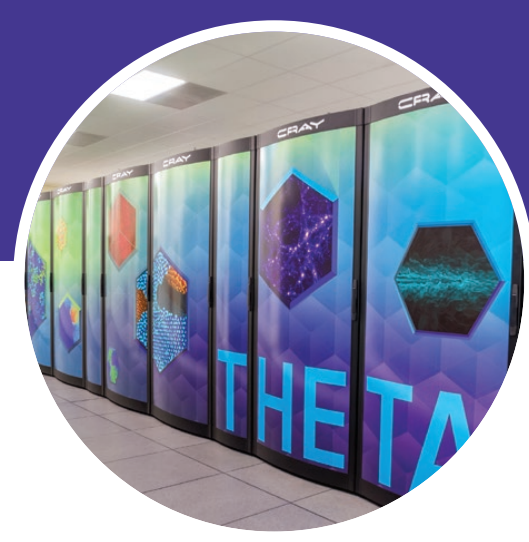

\section{THETA}

Theta, a 9.65-petaflops

supercomputer, serves as a bridge between Mira and the ALCF's next leadership-class system, Aurora. Theta will be used for computational science and engineering research, while providing an early production system to help ALCF users transition their applications to the new Cray/ Intel ${ }^{\circledR}$ Xeon Phi ${ }^{\mathrm{TM}}$ architecture.

$\square$ Cray/Intel ${ }^{\circledR}$ Xeon Phi ${ }^{\text {TM }}$ architecture

$\square 9.65$ petaflops

$\square$ 64-core $1.3 \mathrm{GHz}$ Intel 7230 processor per node

$\square$ 3,624 nodes

$\square 231,935$ cores

$\square 679$ TB of memory

$\square 56$ TB of high-bandwidth memory

$\square$ Aries interconnect with Dragonfly configuration

$\square 20$ racks

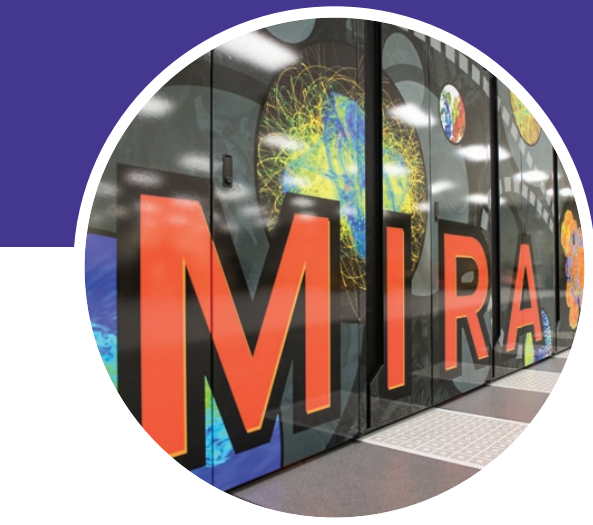

\section{MIRA}

Mira is the ALCF's 10-petaflops IBM Blue Gene/Q supercomputer. In addition to being one of the most powerful supercomputers in the world, Mira is also among the most energy efficient. The system saves considerable energy through innovative chip designs and a unique water-cooling system.
$\square$ IBM Blue Gene/Q architecture
$\square 10$ petaflops
$\square$ 16-core 1.6 GHz IBM PowerPC A2 processor per node
$\square$ 49,152 nodes
$\square 786,432$ cores
$\square 768$ TB of memory
$\square$ 5D torus interconnect
$\square 48$ racks

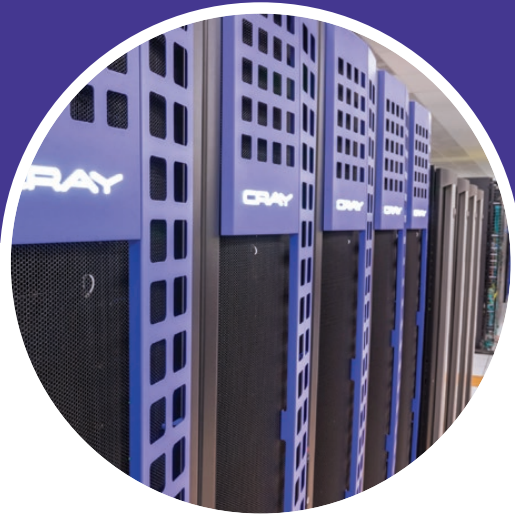

\section{COOLEY}

Cooley is the ALCF's data analysis and visualization cluster. The system helps researchers analyze and explore the massive datasets that result from their simulations on Mira. Cooley shares file systems with the supercomputer, enabling direct access to Mira-generated results.

$\square$ Intel ${ }^{\circledR}$ Haswell architecture

$\square 293$ teraflops

$\square 2$ 6-core 2.4 GHz Intel E5-2620 processors per node

$\square 1$ NVIDIA Tesla K80 GPU per node

$\square 126$ nodes

$\square 1,512$ cores

$\square 47$ TB of memory

$\square 3$ TB of GPU memory

$\square$ FDR Infiniband interconnect

$\square 6$ racks 


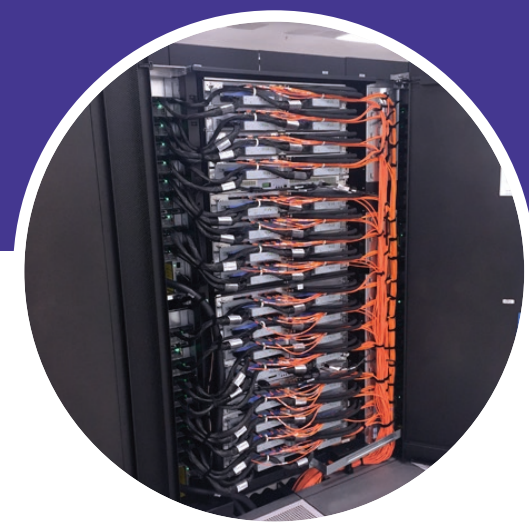

\section{CETUS}

Cetus is an IBM Blue Gene/Q system used to offload both debugging issues and alternative production workloads from Mira. To facilitate these activities, Cetus shares the same software environment and file systems as Mira.

$\square$ IBM Blue Gene/Q architecture

$\square 838$ teraflops

$\square$ 16-core 1.6 GHz IBM PowerPC A2 processor per node

$\square$ 4,096 nodes

$\square 65,536$ cores

$\square 64$ TB of memory

$\square$ 5D torus interconnect

$\square 4$ racks

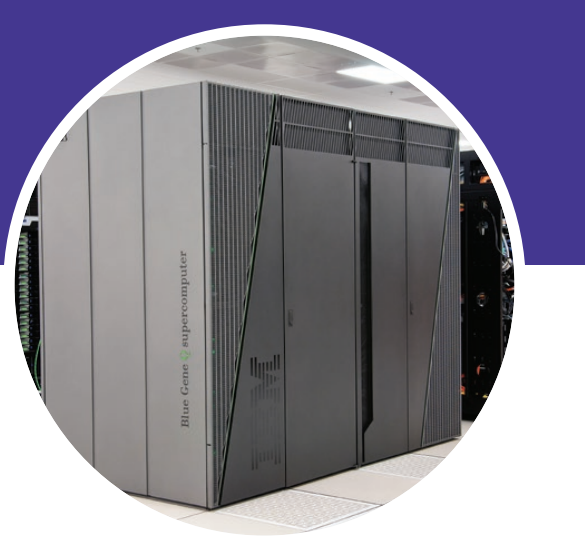

VESTA

Vesta, an IBM Blue Gene/Q system, serves at the ALCF's test and development platform, providing a computing resource for researchers preparing to use Mira.

$\square$ IBM Blue Gene/Q architecture

$\square 419$ teraflops

$\square$ 16-core 1.6 GHz IBM PowerPC A2 processor per node

$\square$ 2,048 nodes

$\square$ 32,768 cores

$\square 32$ TB of memory

$\square$ 5D torus interconnect

$\square 2$ racks

\section{DATA STORAGE}

At the ALCF, disk storage provides intermediate-term storage for active projects, offering a means to access, analyze, and share simulation results. Tape storage is used to archive data from completed projects.

DISK STORAGE The Blue Gene/Q data systems consist of $384 \mathrm{I} / \mathrm{O}$ nodes that connect to 22 storage arrays that control 13,000 disk drives with a total useable capacity of 27 PB and a maximum aggregate transfer speed of 330 $\mathrm{GB} / \mathrm{s}$ over two file systems. The ALCF uses the General Parallel File System to access the storage.

TAPE STORAGE The ALCF has three 10,000-slot libraries using LTO 6 tape technology. The LTO tape drives have built-in hardware compression with compression ratios typically between 1.25:1 and $2: 1$, depending on the data, giving an effective capacity of 36-60 PB.

\section{NETWORKING}

The Blue Gene/Q systems have an internal proprietary network for communicating between nodes. InfiniBand enables communication between the $1 / 0$ nodes and the storage system. Ethernet is used for external user access, and for maintenance and management of the systems.

The ALCF connects to other research institutions using up to $100 \mathrm{~Gb} / \mathrm{s}$ of network connectivity. Scientists can transfer datasets to and from other institutions over fast research networks, such as the Energy Science Network (ESNet) and Internet2. 
APPENDIX I: ALCF PUBLICATIONS 
Researchers who use ALCF resources are major contributors to numerous publications that document their breakthrough science and engineering. The refereed journal articles and conference proceedings represent research ventures undertaken at the ALCF through programs supported by the U.S. Department of Energy and Argonne National Laboratory.

In 2016, research at the ALCF resulted in more than 250 publications. The following list is ordered by publication month. An asterisk after a name indicates an ALCF author. ALCF publications are listed online at: alcf.anl.gov/publications.

\section{JANUARY}

Bailey, Jon A., A. Bazavov, C. Bernard, C. M. Bouchard, C. DeTar, Daping Du, A. X. El-Khadra, J. Foley, E. D. Freeland, E. Gámiz, Steven Gottlieb, U. M. Heller, R. D. Jain, J. Komijani, A. S. Kronfeld, J. Laiho, L. Levkova, Yuzhi Liu, P. B. Mackenzie, Y. Meurice, E. T. Neil, Si-Wei Qiu, J. N. Simone, R. Sugar, D. Toussaint, R. S. Van de Water, and Ran Zhou. "B $\left.\rightarrow \mathrm{KI}^{+}\right|^{-}$decay Form Factors from Three-Flavor Lattice QCD." Physical Review D 93, no. 2 (January 2016). doi:10.1103/ physrevd.93.025026.

Blum, Thomas, Norman Christ, Masashi Hayakawa, Taku Izubuchi, Luchang Jin, and Christoph Lehner. "Lattice Calculation of Hadronic Light-by-Light Contribution to the Muon Anomalous Magnetic Moment." Physical Review D 93, no. 1 (January 2016). doi:10.1103/physrevd.93.014503.

Bui, Huy, Eun-Sung Jung, Venkatram Vishwanath,* Andrew Johnson, Jason Leigh, and Michael E. Papka.* "Improving Sparse Data Movement Performance Using Multiple Paths on the Blue Gene/Q Supercomputer." Paralle/ Computing 51 (January 2016): 3-16. doi:10.1016/j.parco.2015.09.002.

Burstedde, Carsten, and Johannes Holke. "A Tetrahedral Space-Filling Curve for Nonconforming Adaptive Meshes." SIAM Journal on Scientific Computing 38, no. 5 (January 2016): C47-C503. doi:10.1137/15m1040049.

Chen, Jie, Lois C. Mclnnes, and Hong Zhang. "Analysis and Practical Use of Flexible BiCGStab." Journal of Scientific Computing 68, no. 2 (January 2016): 803-825. doi:10.1007/ s10915-015-0159-4.

Dinan, James, Pavan Balaji, Darius Buntinas, David Goodell, William Gropp, and Rajeev Thakur. "An Implementation and Evaluation of the MPI 3.0 One-Sided Communication Interface." Concurrency and Computation: Practice and Experience 28, no. 17 (January 2016): 4385-4404. doi:10.1002/cpe.3758.
Gyulassy, Attila, Aaron Knoll, Kah Chun Lau, Bei Wang, Peer-Timo Bremer, Michael E. Papka,, Larry A. Curtiss, and Valerio Pascucci. "Interstitial and Interlayer Ion Diffusion Geometry Extraction in Graphitic Nanosphere Battery Materials." IEEE Transactions on Visualization and Computer Graphics 22, no. 1 (January 2016): 916-925. doi:10.1109/ tvcg.2015.2467432.

Habib, Salman, Adrian Pope, ${ }^{*}$ Hal Finkel," Nicholas Frontiere, Katrin Heitmann, David Daniel, Patricia Fasel, Vitali Morozov,* George Zagaris, Tom Peterka, Venkatram Vishwanath,* Zarija Lukić, Saba Sehrish, and Wei-keng Liao. "HACC: Simulating Sky Surveys on State-of-the-Art Supercomputing Architectures." New Astronomy 42 (January 2016): 49-65. doi:10.1016/j.newast.2015.06.003.

Harrison, Robert J., Gregory Beylkin, Florian A. Bischoff, Justus A. Calvin, George I. Fann, Jacob Fosso-Tande, Diego Galindo, Jeff R. Hammond, Rebecca Hartman-Baker, Judith C. Hill, Jun Jia, Jakob S. Kottmann, M-J. Yvonne Ou, Junchen Pei, Laura E. Ratcliff," Matthew G. Reuter, Adam C. Richie-Halford, Nichols A. Romero,* Hideo Sekino, William A. Shelton, Bryan E. Sundahl, W. Scott Thornton, Edward F. Valeev, Álvaro Vázquez-Mayagoitia,* Nicholas Vence, Takeshi Yanai, and Yukina Yokoi. "MADNESS: A Multiresolution, Adaptive Numerical Environment for Scientific Simulation." SIAM Journal on Scientific Computing 38, no. 5 (January 2016): S123-S142. doi:10.1137/15m1026171.

Jessup, Elizabeth, Pate Motter, Boyana Norris, and Kanika Sood. "Performance-Based Numerical Solver Selection in the Lighthouse Framework." SIAM Journal on Scientific Computing 38, no. 5 (January 2016): S750-S771. doi:10.1137/15m1028406. 


\section{ALCF PUBLICATIONS CONTINUED}

Maris, P., S. Binder, A. Calci, E. Epelbaum, R. J. Furnstahl, J. Golak, K. Hebeler, H. Kamada, H. Krebs, J. Langhammer, S. Liebig, U.-G. Meißner, D. Minossi, A. Nogga, H. Potter, R. Roth, R. Skibiński, K. Topolnicki, J. P. Vary, and H. Witala. "Properties of $4 \mathrm{He}$ and $6 \mathrm{Li}$ with Improved Chiral EFT Interactions." Edited by C. Elster, D. R. Phillips, and C. D. Roberts. EPJ Web of Conferences 113 (January 2016): 04015. doi:10.1051/epjconf/201611304015.

Maroudas, Dimitrios, Sophie Blondel, Lin Hu, Karl D. Hammond, and Brian D. Wirth. "Helium Segregation on Surfaces of Plasma-Exposed Tungsten." Journal of Physics: Condensed Matter 28, no. 6 (January 2016): 064004. doi:10.1088/0953-8984/28/6/064004.

Melton, Cody A., and Lubos Mitas. "Fixed-Node and Fixed-Phase Approximations and Their Relationship to Variable Spins in Quantum Monte Carlo." Recent Progress in Quantum Monte Carlo (January 2016): 1-13. doi:10.1021/bk2016-1234.ch001.

Niu, Qingpeng, James Dinan, Sravya Tirukkovalur, Anouar Benali,* Jeongnim Kim, Lubos Mitas, Lucas Wagner, and P. Sadayappan. "Global-View Coefficients: A Data Management Solution for Parallel Quantum Monte Carlo Applications." Concurrency and Computation: Practice and Experience 28, no. 13 (January 2016): 3655-3671. doi:10.1002/cpe.3748.

Pan, Kuo-Chuan, Matthias Liebendörfer, Matthias Hempel, and Friedrich-Karl Thielemann. "Two-Dimensional Core-Collapse Supernova Simulations with the Isotropic Diffusion Source Approximation for Neutrino Transport." The Astrophysical Journal 817, no. 1 (January 2016): 72. doi:10.3847/0004-637x/817/1/72.

Perdikaris, Paris, Daniele Venturi, and George Em Karniadakis. "Multifidelity Information Fusion Algorithms for High-Dimensional Systems and Massive Data Sets." SIAM Journal on Scientific Computing 38, no. 4 (January 2016): B521-B538. doi:10.1137/15m1055164.

Peterka, Tom, Hadrien Croubois, Nan Li, Esteban Rangel, and Franck Cappello. "Self-Adaptive Density Estimation of Particle Data." SIAM Journal on Scientific Computing 38, no. 5 (January 2016): S646-S666. doi:10.1137/15m1016308.

Sadhukhan, Jhilam, Witold Nazarewicz, and Nicolas Schunck. "Microscopic Modeling of Mass and Charge Distributions in the Spontaneous Fission of Pu240." Physical Review C 93, no. 1 (January 2016). doi:10.1103/ physrevc.93.011304.
Satuła, Wojciech, and Witold Nazarewicz. "Isospin Effects in $\mathrm{N} \approx Z$ Znuclei in Extended Density Functional Theory." Physica Scripta 91, no. 2 (January 2016): 023013. doi:10.1088/00318949/91/2/023013.

Schatz, Martin D., Robert A. van de Geijn, and Jack Poulson. "Parallel Matrix Multiplication: A Systematic Journey." SIAM Journal on Scientific Computing 38, no. 6 (January 2016): C748-C781. doi:10.1137/140993478.

Skibiński, R., J. Golak, K. Topolnicki, and H. Witała. "N3LO Chiral Predictions for Spin Observables in NucleonDeuteron Elastic Scattering at Low Energies." International Journal of Modern Physics: Conference Series 40 (January 2016): 1660069. doi:10.1142/s2010194516600697.

Yazdani, Alireza, Mingge Deng, Bruce Caswell, and George Em Karniadakis. "Flow in Complex Domains Simulated by Dissipative Particle Dynamics Driven by Geometry-Specific Body-Forces." Journal of Computational Physics 305 (January 2016): 906-920. doi:10.1016/j.jcp.2015.11.001.

\section{FEBRUARY}

Barnes, Tiffany, and George K. Thiruvathukal. "The Need for Research in Broadening Participation." Communications of the ACM 59, no. 3 (February 2016): 33-34. doi:10.1145/2880177.

Boyd, W., A. Siegel, S. He, B. Forget, and K. Smith. "Parallel Performance Results for the OpenMOC Neutron Transport Code on Multicore Platforms." International Journal of High Performance Computing Applications 30, no. 3 (February 2016): 360-375. doi:10.1177/1094342016630388.

Carlsson, B. D., A. Ekström, C. Forssén, D. Fahlin Strömberg, G. R. Jansen, O. Lilja, M. Lindby, B. A. Mattsson, and K. A. Wendt. "Uncertainty Analysis and Order-by-Order Optimization of Chiral Nuclear Interactions." Physical Review X 6, no. 1 (February 2016). doi:10.1103/ physrevx.6.011019.

Chertkov, Eli, Robert A. DiStasio, Ge Zhang, Roberto Car, and Salvatore Torquato. "Inverse Design of Disordered Stealthy Hyperuniform Spin Chains." Physical Review B 93, no. 6 (February 2016). doi:10.1103/physrevb.93.064201.

Colquhoun, B., R. J. Dowdall, J. Koponen, C. T. H. Davies, and G. P. Lepage. "B $\rightarrow \Pi P v$ at Zero Recoil from Lattice QCD with Physical u/d Quarks." Physical Review D 93, no. 3 (February 2016). doi:10.1103/physrevd.93.034502. 
DePrince III, A. Eugene, Jeff R. Hammond, and C. David Sherrill. "Iterative Coupled-Cluster Methods on Graphics Processing Units." Electronic Structure Calculations on Graphics Processing Units (February 2016): 279-300. doi:10.1002/9781118670712.ch13.

Featherstone, Nicholas A., and Bradley W. Hindman. "The Spectral Amplitude of Stellar Convection and Its Scaling in the High-Rayleigh-Number Regime." The Astrophysical Journal 818, no. 1 (February 2016): 32. doi:10.3847/0004$637 \times / 818 / 1 / 32$

Gallandi, Lukas, Noa Marom, Patrick Rinke, and Thomas Körzdörfer. "Accurate Ionization Potentials and Electron Affinities of Acceptor Molecules II: Non-Empirically Tuned Long-Range Corrected Hybrid Functionals." Journal of Chemical Theory and Computation 12, no. 2 (February 2016): 605-614. doi:10.1021/acs.jctc.5b00873.

Garcia Ruiz, R. F., M. L. Bissell, K. Blaum, A. Ekström, N. Frömmgen, G. Hagen, M. Hammen, K. Hebeler, J. D. Holt, G. R. Jansen, M. Kowalska, K. Kreim, W. Nazarewicz, R. Neugart, G. Neyens, W. Nörtershäuser, T. Papenbrock, J. Papuga, A. Schwenk, J. Simonis, K. A. Wendt, and D. T. Yordanov. "Unexpectedly Large Charge Radii of Neutron-Rich Calcium Isotopes." Nature Physics 12, no. 6 (February 2016): 594-598. doi:10.1038/nphys3645.

Knight, Joseph W., Xiaopeng Wang, Lukas Gallandi, Olga Dolgounitcheva, Xinguo Ren, J. Vincent Ortiz, Patrick Rinke, Thomas Körzdörfer, and Noa Marom. "Accurate Ionization Potentials and Electron Affinities of Acceptor Molecules III: A Benchmark of GW Methods." Journal of Chemical Theory and Computation 12, no. 2 (February 2016): 615-626. doi:10.1021/acs.jctc.5b00871.

Kodavasal, Janardhan, Kevin Harms, ${ }^{*}$ Priyesh Srivastava, Sibendu Som, Shaoping Quan, Keith Richards, and Marta García.* "Development of a Stiffness-Based Chemistry Load Balancing Scheme, and Optimization of Input/Output and Communication, to Enable Massively Parallel High-Fidelity Internal Combustion Engine Simulations." Journal of Energy Resources Technology 138, no. 5 (February 2016): 052203. doi:10.1115/1.4032623.

Otten, M., J. Gong, A. Mametjanov, A. Vose, J. Levesque, P. Fischer, and M. Min. "An MPI/OpenACC Implementation of a High-Order Electromagnetics Solver with GPUDirect Communication." International Journal of High Performance Computing Applications 30, no. 3 (February 2016): 320-334. doi:10.1177/1094342015626584.
Perdikaris, Paris, Leopold Grinberg, and George Em Karniadakis. "Multiscale Modeling and Simulation of Brain Blood Flow." Physics of Fluids 28, no. 2 (February 2016): 021304. doi:10.1063/1.4941315.

Richard, Ryan M., Michael S. Marshall, O. Dolgounitcheva, J. V. Ortiz, Jean-Luc Brédas, Noa Marom, and C. David Sherrill. "Accurate Ionization Potentials and Electron Affinities of Acceptor Molecules I. Reference Data at the CCSD(T) Complete Basis Set Limit." Journal of Chemical Theory and Computation 12, no. 2 (February 2016): 595-604. doi:10.1021/ acs.jctc.5b00875.

Savage, John, and Gregory A. Voth. "Proton Solvation and Transport in Realistic Proton Exchange Membrane Morphologies." The Journal of Physical Chemistry C 120, no. 6 (February 2016): 3176-3186. doi:10.1021/acs. jpcc.5b11168.

Seo, Hosung, Marco Govoni, and Giulia Galli. "Design of Defect Spins in Piezoelectric Aluminum Nitride for SolidState Hybrid Quantum Technologies." Scientific Reports 6 (February 2016): 20803. doi:10.1038/srep20803.

Wang, Jiali, Yuefeng Han, Michael L. Stein, Veerabhadra R. Kotamarthi, and Whitney K. Huang. "Evaluation of Dynamically Downscaled Extreme Temperature Using a Spatially-Aggregated Generalized Extreme Value (GEV) Model." Climate Dynamics 47, no. 9-10 (February 2016): 2833-2849. doi:10.1007/s00382-016-3000-3.

Zarzycki, Colin M., Kevin A. Reed, Julio T. Bacmeister, Anthony P. Craig, Susan C. Bates, and Nan A. Rosenbloom. "Impact of Surface Coupling Grids on Tropical Cyclone Extremes in High-Resolution Atmospheric Simulations." Geoscientific Model Development 9, no. 2 (February 2016): 779-788. doi:10.5194/gmd-9-779-2016.

\section{MARCH}

Aad, G., B. Abbott, J. Abdallah, O. Abdinov, B. Abeloos, R. Aben, ATLAS Collaboration, et al. "Search for New Phenomena with Photon+jet Events in Proton-Proton Collisions at $\sqrt{ } \mathrm{s}=13 \mathrm{TeV}$ with the ATLAS Detector." Journal of High Energy Physics 2016, no. 3 (March 2016). doi:10.1007/ jhep03(2016)041.

Aad, G., B. Abbott, J. Abdallah, O. Abdinov, B. Abeloos, R. Aben, M. Abolins, ATLAS Collaboration, et al. "Measurement of the ZZ Production Cross Section in pp Collisions at $\sqrt{ } \mathrm{s}=13 \mathrm{TeV}$ with the ATLAS Detector." Physical Review Letters 116, no. 10 (March 2016). doi:10.1103/ physrevlett.116.101801. 


\section{ALCF PUBLICATIONS CONTINUED}

Ambrosetti, A., N. Ferri, R. A. DiStasio, and A. Tkatchenko. "Wavelike Charge Density Fluctuations and van Der Waals Interactions at the Nanoscale." Science 351, no. 6278 (March 2016): 1171-1176. doi:10.1126/science.aae0509.

Bian, Xin, Mingge Deng, Yu-Hang Tang, and George Em Karniadakis. "Analysis of Hydrodynamic Fluctuations in Heterogeneous Adjacent Multidomains in Shear Flow." Physical Review E 93, no. 3 (March 2016). doi:10.1103/ physreve.93.033312.

Boyle, P. A., N. H. Christ, N. Garron, C. Jung, A. Jüttner, C. Kelly, R. D. Mawhinney, G. McGlynn, D. J. Murphy, S. Ohta, A. Portelli, and C. T. Sachrajda. "Low Energy Constants of SU(2) Partially Quenched Chiral Perturbation Theory from $\mathrm{N}_{\mathrm{f}}=2+1$ Domain Wall QCD." Physical Review D 93, no. 5 (March 2016). doi:10.1103/physrevd.93.054502.

DeJaco, Robert F., Peng Bai, Michael Tsapatsis, and J. Ilja Siepmann. "Adsorptive Separation of 1-Butanol from Aqueous Solutions Using MFI- and FER-Type Zeolite Frameworks: A Monte Carlo Study." Langmuir 32, no. 8 (March 2016): 2093-2101. doi:10.1021/acs.langmuir.5b04483.

Heitmann, Katrin, Derek Bingham, Earl Lawrence, Steven Bergner, Salman Habib, David Higdon, Adrian Pope,* Rahul Biswas, Hal Finkel," Nicholas Frontiere, and Suman Bhattacharya. "The Mira-Titan Universe: Precision Predictions For Dark Energy Surveys.” The Astrophysical Journal 820, no. 2 (March 2016): 108. doi:10.3847/0004$637 \times / 820 / 2 / 108$.

Ibeid, H., R. Yokota, and D. Keyes. "A Performance Model for the Communication in Fast Multipole Methods on HighPerformance Computing Platforms." International Journal of High Performance Computing Applications 30, no. 4 (March 2016): 423-437. doi:10.1177/1094342016634819.

Lovato, A., O. Benhar, J. Carlson, S. Gandolfi, Steven C. Pieper, N. Rocco, and R. Schiavilla. "Ab Initio Calculation of the Electromagnetic and Neutral-Weak Response Functions of ${ }^{4} \mathrm{He}$ and ${ }^{12} \mathrm{C}$." Edited by C. Elster, D. R. Phillips, and C. D. Roberts. EPJ Web of Conferences 113 (March 2016): 01010. doi:10.1051/epjconf/201611301010.

Maffeo, Christopher, Jejoong Yoo, and Aleksei Aksimentiev. "De Novo Reconstruction of DNA Origami Structures through Atomistic Molecular Dynamics Simulation." Nucleic Acids Research 44, no. 7 (March 2016): 3013-3019. doi:10.1093/nar/gkw155.
Maleki, Saeed, Donald Nguyen, Andrew Lenharth, María Garzarán, David Padua, and Keshav Pingali. "DSMR." Proceedings of the 21st ACM SIGPLAN Symposium on Principles and Practice of Parallel Programming - PPoPP '16 (March 2016). doi:10.1145/2851141.2851183.

Martin, D., A. Arcones, W. Nazarewicz, and E. Olsen. "Impact of Nuclear Mass Uncertainties on the r Process." Physical Review Letters 116, no. 12 (March 2016). doi:10.1103/ physrevlett.116.121101.

Nazarewicz, W. "Challenges in Nuclear Structure Theory." Journal of Physics G: Nuclear and Particle Physics 43, no. 4 (March 2016): 044002. doi:10.1088/0954-3899/43/4/044002.

Reda, Khairi, Andrew E. Johnson, Michael E. Papka," and Jason Leigh. "Modeling and Evaluating User Behavior in Exploratory Visual Analysis." Information Visualization 15, no. 4 (March 2016): 325-339. doi:10.1177/1473871616638546.

Tardieu, Olivier, Wei Zhang, Benjamin Herta, David Cunningham, David Grove, Prabhanjan Kambadur, Vijay Saraswat, Avraham Shinnar, Mikio Takeuchi, and Mandana Vaziri. "X10 and APGAS at Petascale." ACM Transactions on Parallel Computing 2, no. 4 (March 2016): 1-32. doi:10.1145/2894746.

Totorica, Samuel R., Tom Abel, and Frederico Fiuza. "Nonthermal Electron Energization from Magnetic Reconnection in Laser-Driven Plasmas." Physical Review Letters 116, no. 9 (March 2016). doi:10.1103/ physrevlett.116.095003.

Williams, Timothy J.* "Delivering Science on Day One." Computing in Science \& Engineering 18, no. 2 (March 2016): 104-107. doi:10.1109/mcse.2016.22.

\section{APRIL}

Arntsen, C., J. Savage, Y.-L. S. Tse, and G. A. Voth. "Simulation of Proton Transport in Proton Exchange Membranes with Reactive Molecular Dynamics." Fuel Cells 16, no. 6 (April 2016): 695-703. doi:10.1002/fuce.201600024.

Bianchini, F., J. R. Kermode, and A. De Vita. "Modelling Defects in N-Al with EAM and DFT Calculations." Modelling and Simulation in Materials Science and Engineering 24, no. 4 (April 2016): 045012. doi:10.1088/09650393/24/4/045012. 
Binder, S., A. Calci, E. Epelbaum, R. J. Furnstahl, J. Golak, K. Hebeler, H. Kamada, H. Krebs, J. Langhammer, S. Liebig, P. Maris, Ulf-G. Meißner, D. Minossi, A. Nogga, H. Potter, R. Roth, R. Skibiński, K. Topolnicki, J. P. Vary, H. Witała, and the LENPIC Collaboration. "Few-Nucleon Systems with State-of-the-Art Chiral Nucleon-Nucleon Forces." Physical Review C 93, no. 4 (April 2016). doi:10.1103/ physrevc.93.044002.

Binder, S., A. Ekström, G. Hagen, T. Papenbrock, and K. A. Wendt. "Effective Field Theory in the Harmonic Oscillator Basis." Physical Review C 93, no. 4 (April 2016). doi:10.1103/ physrevc.93.044332.

Bois, Juliana, and Thomas Körzdörfer. "How Bond Length Alternation and Thermal Disorder Affect the Optical Excitation Energies of $\pi$-Conjugated Chains: A Combined Density Functional Theory and Molecular Dynamics Study." Journal of Chemical Theory and Computation 12, no. 4 (April 2016): 1872-1882. doi:10.1021/acs.jctc.5b01070.

Bosler, Peter A., Erika L. Roesler, Mark A. Taylor, and Miranda R. Mundt. "Stride Search: A General Algorithm for Storm Detection in High-Resolution Climate Data." Geoscientific Model Development 9, no. 4 (April 2016): 1383-1398. doi:10.5194/gmd-9-1383-2016.

Boughezal, Radja, John Campbell, R. Keith Ellis, Christfried Focke, Walter Giele, Xiaohui Liu, and Frank Petriello. "Z-Boson Production in Association with a Jet at Next-To-Next-To-Leading Order in Perturbative QCD." Physical Review Letters 116, no. 15 (April 2016). doi:10.1103/ physrevlett.116.152001.

Gillan, Michael J., Dario Alfè, and Angelos Michaelides. "Perspective: How Good Is DFT for Water?" The Journal of Chemical Physics 144, no. 13 (April 2016): 130901. doi:10.1063/1.4944633.

Hager, Robert, and C. S. Chang. "Gyrokinetic Neoclassical Study of the Bootstrap Current in the Tokamak Edge Pedestal with Fully Non-Linear Coulomb Collisions." Physics of Plasmas 23, no. 4 (April 2016): 042503. doi:10.1063/1.4945615.

Hinohara, Nobuo, and Witold Nazarewicz. "Pairing NambuGoldstone Modes Within Nuclear Density Functional Theory." Physical Review Letters 116, no. 15 (April 2016). doi:10.1103/physrevlett.116.152502.
Kodavasal, Janardhan, Yuanjiang Pei, Kevin Harms, ${ }^{*}$ Stephen Ciatti, Al Wagner, Peter Senecal, Marta García, ${ }^{*}$ and Sibendu Som. "Global Sensitivity Analysis of a Gasoline Compression Ignition Engine Simulation with Multiple Targets on an IBM Blue Gene/Q Supercomputer." SAE Technical Paper Series (April 2016). doi:10.4271/2016-01-0602.

Leger, Timothy, Nicholas Bisek, and Jonathan Poggie. "Computations of Turbulent Flow over a Sharp Fin at Mach 5." Journal of Thermophysics and Heat Transfer 30, no. 2 (April 2016): 394-402. doi:10.2514/1.t4698.

Li, Ying, Rajiv K. Kalia, Masaaki Misawa, Aiichiro Nakano, Ken-ichi Nomura, Kohei Shimamura, Fuyuki Shimojo, and Priya Vashishta. "Anisotropic Mechanoresponse of Energetic Crystallites: A Quantum Molecular Dynamics Study of Nano-Collision." Nanoscale 8, no. 18 (April 2016): 9714-9720. doi:10.1039/c5nr08769d.

Mubarak, Misbah, Christopher D. Carothers, Robert B. Ross, and Philip Carns. "Enabling Parallel Simulation of Large-

Scale HPC Network Systems." IEEE Transactions on Parallel and Distributed Systems 28, no. 1 (April 2016): 87-100. doi:10.1109/tpds.2016.2543725.

Navrátil, Petr, Sofia Quaglioni, Guillaume Hupin, Carolina Romero-Redondo, and Angelo Calci. "Unified Ab Initio Approaches to Nuclear Structure and Reactions." Physica Scripta 91, no. 5 (April 2016): 053002. doi:10.1088/00318949/91/5/053002.

Nomura, Ken-ichi, Rajiv K. Kalia, Ying Li, Aiichiro Nakano, Pankaj Rajak, Chunyang Sheng, Kohei Shimamura, Fuyuki Shimojo, and Priya Vashishta. "Nanocarbon Synthesis by High-Temperature Oxidation of Nanoparticles." Scientific Reports 6 (April 2016): 24109. doi:10.1038/srep24109.

Offermans, Nicolas, Oana Marin, Michel Schanen, Jing Gong, Paul Fischer, and Philipp Schlatter. "On the Strong Scaling of the Spectral Element Solver Nek5000 on Petascale Systems." Proceedings of the Exascale Applications and Software Conference 2016 - EASC '16 (April 2016). doi:10.1145/2938615.2938617.

Packwood, David, James Kermode, Letif Mones, Noam Bernstein, John Woolley, Nicholas Gould, Christoph Ortner, and Gábor Csányi. "A Universal Preconditioner for Simulating Condensed Phase Materials." The Journal of Chemical Physics 144, no. 16 (April 2016): 164109. doi:10.1063/1.4947024. 


\section{ALCF PUBLICATIONS CONTINUED}

Pruitt, Spencer R., Hiroya Nakata, Takeshi Nagata, Maricris Mayes, Yuri Alexeev,* Graham Fletcher,* Dmitri G. Fedorov, Kazuo Kitaura, and Mark S. Gordon. "Importance of ThreeBody Interactions in Molecular Dynamics Simulations of Water Demonstrated with the Fragment Molecular Orbital Method." Journal of Chemical Theory and Computation 12, no. 4 (April 2016): 1423-1435. doi:10.1021/acs.jctc.5b01208.

Sai Gautam, Gopalakrishnan, Pieremanuele Canepa, William Davidson Richards, Rahul Malik, and Gerbrand Ceder. "Role of Structural H2O in Intercalation Electrodes: The Case of Mg in Nanocrystalline Xerogel-V2O5." Nano Letters 16, no. 4 (April 2016): 2426-2431. doi:10.1021/acs.nanolett.5b05273.

Shah, Mansi S., Michael Tsapatsis, and J. Ilja Siepmann. "Identifying Optimal Zeolitic Sorbents for Sweetening of Highly Sour Natural Gas." Angewandte Chemie International Edition 55, no. 20 (April 2016): 5938-5942. doi:10.1002/ anie. 201600612 .

Tang, Yu-Hang, Zhen Li, Xuejin Li, Mingge Deng, and George Em Karniadakis. "Non-Equilibrium Dynamics of Vesicles and Micelles by Self-Assembly of Block Copolymers with Double Thermoresponsivity." Macromolecules 49, no. 7 (April 2016): 2895-2903. doi:10.1021/acs.macromol.6b00365.

Yazdani, Alireza, and George Em Karniadakis. "Sub-Cellular Modeling of Platelet Transport in Blood Flow through Microchannels with Constriction." Soft Matter 12, no. 19 (April 2016): 4339-4351. doi:10.1039/c6sm00154h.

\section{MAY}

Aad, G., B. Abbott, J. Abdallah, O. Abdinov, B. Abeloos, R. Aben, ATLAS Collaboration, et al. "Muon Reconstruction Performance of the ATLAS Detector in Proton-Proton Collision Data at $\sqrt{ } \mathrm{s}=13 \mathrm{TeV}$." The European Physical Journal C 76, no. 5 (May 2016). doi:10.1140/epjc/s10052-0164120-y.

Aad, G., B. Abbott, J. Abdallah, O. Abdinov, B. Abeloos, and R. Aben, ATLAS Collaboration, et al. "Search for Supersymmetry at $\sqrt{ } \mathrm{s}=13 \mathrm{TeV}$ in Final States with Jets and Two Same-Sign Leptons or Three Leptons with the ATLAS Detector." The European Physical Journal C 76, no. 5 (May 2016). doi:10.1140/epjc/s10052-016-4095-8.
Bazavov, A., C. Bernard, N. Brown, J. Komijani, C. DeTar, J. Foley, L. Levkova, Steven Gottlieb, U. M. Heller, J. Laiho, R. L. Sugar, D. Toussaint, and R. S. Van de Water (MILC Collaboration). "Gradient Flow and Scale Setting on MILC HISQ Ensembles.” Physical Review D 93, no. 9 (May 2016). doi:10.1103/physrevd.93.094510.

Chatzopoulos, E., Sean M. Couch, W. David Arnett, and F. X. Timmes. "Convective Properties of Rotating TwoDimensional Core-Collapse Supernova Progenitors." The Astrophysical Journal 822, no. 2 (May 2016): 61. doi:10.3847/0004-637x/822/2/61.

Chen, Qile P., Justine D. Chu, Robert F. DeJaco, Timothy P. Lodge, and J. Ilja Siepmann. "Molecular Simulation of Olefin Oligomer Blend Phase Behavior." Macromolecules 49, no. 10 (May 2016): 3975-3985. doi:10.1021/acs.macromol.6b00394.

Fedorov, Dmitry A., Spencer R. Pruitt, Kristopher Keipert, Mark S. Gordon, and Sergey A. Varganov. "Ab Initio Multiple Spawning Method for Intersystem Crossing Dynamics: SpinForbidden Transitions between ${ }^{3} \mathrm{~B}_{1}$ and ${ }^{1} \mathrm{~A}_{1}$ States of $\mathrm{GeH}_{2}$." The Journal of Physical Chemistry A 120, no. 18 (May 2016): 2911-2919. doi:10.1021/acs.jpca.6b01406.

Flender, Samuel, Lindsey Bleem, Hal Finkel,* Salman Habib, Katrin Heitmann, and Gilbert Holder. "Simulations of the Pairwise Kinematic Sunyaev-Zel'dovich Signal." The Astrophysical Journal 823, no. 2 (May 2016): 98. doi:10.3847/0004-637x/823/2/98.

Hagen, G., M. Hjorth-Jensen, G. R. Jansen, and T. Papenbrock. "Emergent Properties of Nuclei from Ab Initio Coupled-Cluster Calculations." Physica Scripta 91, no. 6 (May 2016): 063006. doi:10.1088/0031-8949/91/6/063006.

Ibanez, Daniel A., E. Seegyoung Seol, Cameron W. Smith, and Mark S. Shephard. "PUMI." ACM Transactions on Mathematical Software 42, no. 3 (May 2016): 1-28. doi:10.1145/2814935.

Isaila, Florin, Jesus Carretero, and Rob Ross. "CLARISSE: A Middleware for Data-Staging Coordination and Control on Large-Scale HPC Platforms." 2016 16th IEEE/ACM International Symposium on Cluster, Cloud, and Grid Computing (CCGrid) (May 2016). doi:10.1109/ccgrid.2016.24.

Landry, Walter, and Sylvain Barbot. "Gamra: Simple Meshing for Complex Earthquakes." Computers \& Geosciences 90 (May 2016): 49-63. doi:10.1016/j.cageo.2016.02.014. 
Lee, En-Jui, and Po Chen. "Improved Basin Structures in Southern California Obtained Through Full-3D Seismic Waveform Tomography (F3DT)." Seismological Research Letters 87, no. 4 (May 2016): 874-881. doi:10.1785/0220160013.

Li, Jie, and Yung Y. Liu. "Thermal Modeling of a Vertical Dry Storage Cask for Used Nuclear Fuel.” Nuclear Engineering and Design 301 (May 2016): 74-88. doi:10.1016/j. nucengdes.2016.01.008.

Lu, Lu, Xuejin Li, Peter G. Vekilov, and George Em Karniadakis. "Probing the Twisted Structure of Sickle Hemoglobin Fibers via Particle Simulations." Biophysical Journal 110, no. 9 (May 2016): 2085-2093. doi:10.1016/j. bpj.2016.04.002.

Matsui, Hiroaki, Eric Heien, Julien Aubert, Jonathan M. Aurnou, Margaret Avery, Ben Brown, Bruce A. Buffett, et al. "Performance Benchmarks for a Next Generation Numerical Dynamo Model." Geochemistry, Geophysics, Geosystems 17, no. 5 (May 2016): 1586-1607. doi:10.1002/2015gc006159.

Mikida, Eric, Nikhil Jain, Laxmikant Kale, Elsa Gonsiorowski, Christopher D. Carothers, Peter D. Barnes, and David Jefferson. "Towards PDES in a Message-Driven Paradigm." Proceedings of the 2016 Annual ACM Conference on SIGSIM Principles of Advanced Discrete Simulation - SIGSIM-PADS ‘6 (May 2016). doi:10.1145/2901378.2901393.

Perdikaris, Paris, and George Em Karniadakis. "Model Inversion via Multi-Fidelity Bayesian Optimization: A New Paradigm for Parameter Estimation in Haemodynamics, and Beyond." Journal of The Royal Society Interface 13, no. 118 (May 2016): 20151107. doi:10.1098/rsif.2015.1107.

Raimondi, Francesco, Guillaume Hupin, Petr Navrátil, and Sofia Quaglioni. “Deuteron-Induced Nucleon Transfer Reactions Within An Ab Initio Framework: First Application Top-Shell Nuclei." Physical Review C 93, no. 5 (May 2016). doi:10.1103/physrevc.93.054606.

Regnier, D., N. Dubray, N. Schunck, and M. Verrière. "Fission Fragment Charge and Mass Distributions in Pu239(n,f) in the Adiabatic Nuclear Energy Density Functional Theory." Physical Review C 93, no. 5 (May 2016). doi:10.1103/ physrevc.93.054611.
Rizzi, Silvio,, Mark Hereld, Joseph Insley,* Preeti Malakar,* Michael E. Papka,* Thomas Uram,* and Venkatram Vishwanath.* "Coupling LAMMPS and the VI3 Framework for Co-Visualization of Atomistic Simulations." 2016 IEEE International Parallel and Distributed Processing Symposium Workshops (IPDPSW) (May 2016). doi:10.1109/ ipdpsw.2016.26.

Sagert, I., G. I. Fann, F. J. Fattoyev, S. Postnikov, and C. J. Horowitz. "Quantum Simulations of Nuclei and Nuclear Pasta with the Multiresolution Adaptive Numerical Environment for Scientific Simulations." Physical Review C 93, no. 5 (May 2016). doi:10.1103/physrevc.93.055801.

Schlagkamp, Stephan, Rafael Ferreira da Silva, William Allcock, Ewa Deelman, and Uwe Schwiegelshohn. "Consecutive Job Submission Behavior at Mira Supercomputer." Proceedings of the 25th ACM International Symposium on High-Performance Parallel and Distributed Computing - HPDC '16 (May 2016). doi:10.1145/2907294.2907314.

Schuetrumpf, B., W. Nazarewicz, and P.-G. Reinhard. "TimeDependent Density Functional Theory with Twist-Averaged Boundary Conditions." Physical Review C 93, no. 5 (May 2016). doi:10.1103/physrevc.93.054304.

Uram, Thomas D.,* and Michael E. Papka.* "Expanding the Scope of High-Performance Computing Facilities." Computing in Science \& Engineering 18, no. 3 (May 2016): 84-87. doi:10.1109/mcse.2016.53.

Wu, Xingfu, and Valerie Taylor. "Utilizing Hardware Performance Counters to Model and Optimize the Energy and Performance of Large Scale Scientific Applications on Power-Aware Supercomputers." 2016 IEEE International Parallel and Distributed Processing Symposium Workshops (IPDPSW) (May 2016). doi:10.1109/ipdpsw.2016.78.

Yang, Fan, and Andrew A. Chien. "ZCCloud: Exploring Wasted Green Power for High-Performance Computing." 2016 IEEE International Parallel and Distributed Processing Symposium (IPDPS) (May 2016). doi:10.1109/ipdps.2016.96.

\section{JUNE}

Aad, G., B. Abbott, J. Abdallah, O. Abdinov, B. Abeloos, R. Aben, M. Abolins, ATLAS Collaboration, et al. "Search for New Phenomena in Final States with Large Jet Multiplicities and Missing Transverse Momentum with ATLAS Using $\sqrt{ } \mathrm{s}=13$ TeV Proton-Proton Collisions." Physics Letters B 757 (June 2016): 334-355. doi:10.1016/j.physletb.2016.04.005. 
Bazavov, A., C. Bernard, C. M. Bouchard, C. C. Chang, C. DeTar, Daping Du, A. X. El-Khadra, E. D. Freeland, E. Gámiz, Steven Gottlieb, U. M. Heller, A. S. Kronfeld, J. Laiho, P.B. Mackenzie, E. T. Neil, J. Simone, R. Sugar, D. Toussaint, R. S. Van de Water, Ran Zhou, Fermilab Lattice and MILC Collaborations. "B(s)0-Mixing Matrix Elements from Lattice QCD for the Standard Model and Beyond." Physical Review D 93, no. 11 (June 2016). doi:10.1103/physrevd.93.113016.

Benali, Anouar,* Luke Shulenburger, Jaron T. Krogel, Xiaoliang Zhong, Paul R. C. Kent, and Olle Heinonen. "Quantum Monte Carlo Analysis of a Charge Ordered Insulating Antiferromagnet: The $\mathrm{Ti}_{4} \mathrm{O}_{7}$ Magnéli Phase." Phys. Chem. Chem. Phys. 18, no. 27 (June 2016): 1832318335. doi:10.1039/c6cp02067d.

Blum, T., P. A. Boyle, T. Izubuchi, L. Jin, A. Jüttner, C. Lehner, K. Maltman, M. Marinkovic, A. Portelli, M. Spraggs, RBC and UKQCD Collaborations. "Calculation of the Hadronic Vacuum Polarization Disconnected Contribution to the Muon Anomalous Magnetic Moment." Physical Review Letters 116, no. 23 (June 2016). doi:10.1103/physrevlett.116.232002.

Conway, Patrick, and Frank DiMaio. "Improving Hybrid Statistical and Physical Forcefields through Local Structure Enumeration." Protein Science 25, no. 8 (June 2016): 1525-1534. doi:10.1002/pro.2956.

Dohet-Eraly, Jérémy, Petr Navrátil, Sofia Quaglioni, Wataru Horiuchi, Guillaume Hupin, and Francesco Raimondi.

${ }^{3} \mathrm{He}(\mathrm{a}, \gamma)^{7} \mathrm{Be}$ and $\left.{ }^{3} \mathrm{H}(\mathrm{a}, \gamma)\right)^{7} \mathrm{Li}$ Astrophysical S Factors from the No-Core Shell Model with Continuum." Physics Letters B 757 (June 2016): 430-436. doi:10.1016/j.physletb.2016.04.021.

Dytrych, T., Pieter Maris, K. D. Launey, J. P. Draayer, James Vary, D. Langr, E. Saule, M. A. Caprio, U. Catalyurek, and M. Sosonkina. "Efficacy of the SU(3) Scheme for Ab Initio Large-Scale Calculations Beyond the Lightest Nuclei" (June 2016). doi:10.2172/1326837.

Fetisov, Evgenii O., I-Feng William Kuo, Chris Knight, ${ }^{\text {Joost }}$ VandeVondele, Troy Van Voorhis, and J. Ilja Siepmann. "First-Principles Monte Carlo Simulations of Reaction Equilibria in Compressed Vapors." ACS Central Science 2, no. 6 (June 2016): 409-415. doi:10.1021/acscentsci.6b00095.

Gaiduk, Alex P., Marco Govoni, Robert Seidel, Jonathan H. Skone, Bernd Winter, and Giulia Galli. "Photoelectron Spectra of Aqueous Solutions from First Principles." Journal of the American Chemical Society 138, no. 22 (June 2016): 6912-6915. doi:10.1021/jacs.6b00225.
Hager, Robert, E. S. Yoon, S. Ku, E. F. D’Azevedo, P. H. Worley, and C. S. Chang. "A Fully Non-Linear Multi-Species Fokker-Planck-Landau Collision Operator for Simulation of Fusion Plasma." Journal of Computational Physics 315 (June 2016): 644-660. doi:10.1016/j.jcp.2016.03.064.

Huang, Junji, and Lian Duan. "Turbulent Inflow Generation for Direct Simulations of Hypersonic Turbulent Boundary Layers and Their Freestream Acoustic Radiation." 46th AIAA Fluid Dynamics Conference (June 2016). doi:10.2514/6.20163639.

Hutchinson, Maxwell, Alexander Heinecke, Hans Pabst, Greg Henry, Matteo Parsani, and David Keyes. "Efficiency of High Order Spectral Element Methods on Petascale Architectures." High Performance Computing (June 2016): 449-466. doi:10.1007/978-3-319-41321-1_23.

Jain, Nikhil, Eric Bohm, Eric Mikida, Subhasish Mandal, Minjung Kim, Prateek Jindal, Qi Li, Sohrab Ismail-Beigi, Glenn J. Martyna, and Laxmikant V. Kale. "OpenAtom: Scalable Ab-Initio Molecular Dynamics with Diverse Capabilities." High Performance Computing (June 2016): 139-158. doi:10.1007/978-3-319-41321-1_8.

Kaurov, Alexander A., and Nickolay Y. Gnedin. "Cosmic Reionization on Computers: Mean and Fluctuating Redshifted $21 \mathrm{Cm}$ Signal." The Astrophysical Journal 824, no. 2 (June 2016): 114. doi:10.3847/0004-637x/824/2/114.

Laskowski, Gregory M., James Kopriva, Vittorio Michelassi, Sriram Shankaran, Umesh Paliath, Rathakrishnan Bhaskaran, Qiqi Wang, Chaitanya Talnikar, Zhi J. Wang, and Feilin Jia. "Future Directions of High Fidelity CFD for Aerothermal Turbomachinery Analysis and Design." 46th AIAA Fluid Dynamics Conference (June 2016). doi:10.2514/6.2016-3322.

Liu, Jinxun, Benjamin M. Sleeter, Zhiliang Zhu, Linda S. Heath, Zhengxi Tan, Tamara S. Wilson, Jason Sherba, and Decheng Zhou. "Estimating Carbon Sequestration in the Piedmont Ecoregion of the United States from 1971 to 2010." Carbon Balance and Management 11, no. 1 (June 2016). doi:10.1186/s13021-016-0052-y.

Pivkin, Igor V., Zhangli Peng, George E. Karniadakis, Pierre A. Buffet, Ming Dao, and Subra Suresh. "Biomechanics of Red Blood Cells in Human Spleen and Consequences for Physiology and Disease." Proceedings of the National Academy of Sciences 113, no. 28 (June 2016): 7804-7809. doi:10.1073/pnas.1606751113. 
Skone, Jonathan H., Marco Govoni, and Giulia Galli. "Nonempirical Range-Separated Hybrid Functionals for Solids and Molecules." Physical Review B 93, no. 23 (June 2016). doi:10.1103/physrevb.93.235106.

Soergel, B., S. Flender, K. T. Story, L. Bleem, T. Giannantonio, G. Efstathiou, E. Rykoff, DES and SPT Collaborations, et al. "Detection of the Kinematic Sunyaev-Zel'dovich Effect with DES Year 1 and SPT." Monthly Notices of the Royal Astronomical Society 461, no. 3 (June 2016): 3172-3193. doi:10.1093/mnras/stw1455.

Sung, Yonduck, Anne L. Dord, Gregory M. Laskowski, Lee Shunn, Greg Natsui, and Jay Kapat. "Detailed Large Eddy Simulations (LES) of Multi-Hole Effusion Cooling Flow for Gas Turbines." ASME Turbo Expo 2016: Turbomachinery Technical Conference and Exposition, Volume 5B: Heat Transfer (June 2016). doi:10.1115/gt2016-57957.

Wang, Xiaopeng, Taylor Garcia, Stephen Monaco, Bohdan Schatschneider, and Noa Marom. "Effect of Crystal Packing on the Excitonic Properties of Rubrene Polymorphs." CrystEngComm 18, no. 38 (June 2016): 7353-7362. doi:10.1039/c6ce00873a.

Wermelinger, Fabian, Babak Hejazialhosseini, Panagiotis Hadjidoukas, Diego Rossinelli, and Petros Koumoutsakos. "An Efficient Compressible Multicomponent Flow Solver for Heterogeneous CPU/GPU Architectures." Proceedings of the Platform for Advanced Scientific Computing Conference on ZZZ - PASC '16 (June 2016). doi:10.1145/2929908.2929914.

Zee, Field G. Van, Vernon Austel, John A. Gunnels, Lee Killough, Tyler M. Smith, Bryan Marker, Tze Meng Low, Robert A. Van De Geijn, Francisco D. Igual, Mikhail Smelyanskiy, Xianyi Zhang, and Michael Kistler. "The BLIS Framework." ACM Transactions on Mathematical Software 42, no. 2 (June 2016): 1-19. doi:10.1145/2755561.

\section{JULY}

Abdullah, Syed Umer, Yuri Alexeev, ${ }^{*}$ Philip E. Johnson, Neil M. Rigby, Alan R. Mackie, Balvinder Dhaliwal, and E. N. Clare Mills. "Ligand Binding to an Allergenic Lipid Transfer Protein Enhances Conformational Flexibility Resulting in an Increase in Susceptibility to Gastroduodenal Proteolysis." Scientific Reports 6 (July 2016): 30279. doi:10.1038/srep30279.
Berman, Diana, Sanket A. Deshmukh, Badri Narayanan, Subramanian K. R. S. Sankaranarayanan, Zhong Yan, Alexander A. Balandin, Alexander Zinovev, Daniel Rosenmann, and Anirudha V. Sumant. "Metal-Induced Rapid Transformation of Diamond into Single and Multilayer Graphene on Wafer Scale." Nature Communications 7 (July 2016): 12099. doi:10.1038/ncomms12099.

Bhandari, Yuba R., Wei Jiang, ${ }^{*}$ Eric A. Stahlberg, Jason R. Stagno, and Yun-Xing Wang. "Modeling RNA Topological Structures Using Small Angle X-Ray Scattering." Methods 103 (July 2016): 18-24. doi:10.1016/j. ymeth.2016.04.015.

Borrell, Guillem, and Javier Jiménez. "Properties of the Turbulent/non-Turbulent Interface in Boundary Layers." Journal of Fluid Mechanics 801 (July 2016): 554-596. doi:10.1017/jfm.2016.430.

Borrell, R., J. Chiva, O. Lehmkuhl, G. Oyarzun, I. Rodríguez, and A. Oliva. "Optimising the Termofluids CFD Code for Petascale Simulations." International Journal of Computational Fluid Dynamics 30, no. 6 (July 2016): 425430. doi:10.1080/10618562.2016.1221503.

Busemeyer, Brian, Mario Dagrada, Sandro Sorella, Michele Casula, and Lucas K. Wagner. "Competing Collinear Magnetic Structures in Superconducting FeSe by First-Principles Quantum Monte Carlo Calculations." Physical Review B 94, no. 3 (July 2016). doi:10.1103/ physrevb.94.035108.

Fang, Jun, and Igor A. Bolotnov. "Bubble Tracking Simulations of Turbulent Two-Phase Flows." Volume 1B, Symposia: Fluid Mechanics (Fundamental Issues and Perspectives; Industrial and Environmental Applications); Multiphase Flow and Systems (Multiscale Methods; Noninvasive Measurements; Numerical Methods; Heat Transfer; Performance); Transport Phenomena (Clean Energy; Mixing; Manufacturing and Materials Processing); Turbulent Flows - Issues and Perspectives; Algorithms and Applications for High Performance CFD Computation; Fluid Power; Fluid Dynamics of Wind Energy; Marine Hydrodynamics (July 2016). doi:10.1115/fedsm2016-1005.

Fang, Jun, Michel Rasquin, and Igor A. Bolotnov. "Interface Tracking Simulations of Bubbly Flows in PWR Relevant Geometries." Nuclear Engineering and Design (July 2016). doi:10.1016/j.nucengdes.2016.07.002. 


\section{ALCF PUBLICATIONS CONTINUED}

Fodor, Zoltan, Kieran Holland, Julius Kuti, Santanu Mondal, Daniel Nogradi, and Chik Him Wong. "Electroweak Interactions and Dark Baryons in the Sextet BSM Model with a Composite Higgs Particle." Physical Review D 94, no. 1 (July 2016). doi:10.1103/physrevd.94.014503.

Gnedin, Nickolay Y. "Cosmic Reionization on Computers: The Faint End of the Galaxy Luminosity Function." The Astrophysical Journal 825, no. 2 (July 2016): L17. doi:10.3847/2041-8205/825/2/117.

Hu, B. S., F. R. Xu, Z. H. Sun, J. P. Vary, and T. Li. "Ab Initio Nuclear Many-Body Perturbation Calculations in the Hartree-Fock Basis." Physical Review C 94, no. 1 (July 2016). doi:10.1103/physrevc.94.014303.

Jansen, G. R., M. D. Schuster, A. Signoracci, G. Hagen, and P. Navrátil. "Open sd-Shell Nuclei from First Principles." Physical Review C 94, no. 1 (July 2016). doi:10.1103/ physrevc.94.011301.

Leger, Timothy, Nicholas Bisek, and Jonathan Poggie. "Supersonic Corner Flow Predictions Using the Quadratic Constitutive Relation." AIAA Journal 54, no. 7 (July 2016): 2077-2088. doi:10.2514/1.j054732.

Li, Zhen, Xin Bian, Xiu Yang, and George Em Karniadakis. "A Comparative Study of Coarse-Graining Methods for Polymeric Fluids: Mori-Zwanzig Vs. Iterative Boltzmann Inversion Vs. Stochastic Parametric Optimization." The Journal of Chemical Physics 145, no. 4 (July 2016): 044102. doi:10.1063/1.4959121.

Makarashvili, Vakhtang, Elia Merzari, Aleksandr Obabko, Paul Fischer, and Andrew Siegel. "Accelerating the HighFidelity Simulation of Turbulence: Ensemble Averaging." Volume 1B, Symposia: Fluid Mechanics (Fundamental Issues and Perspectives; Industrial and Environmental Applications); Multiphase Flow and Systems (Multiscale Methods; Noninvasive Measurements; Numerical Methods; Heat Transfer; Performance); Transport Phenomena (Clean Energy; Mixing; Manufacturing and Materials Processing); Turbulent Flows - Issues and Perspectives; Algorithms and Applications for High Performance CFD Computation; Fluid Power; Fluid Dynamics of Wind Energy; Marine Hydrodynamics (July 2016). doi:10.1115/fedsm2016-7853.

Malakar, Preeti,, and Venkatram Vishwanath.* "Data Movement Optimizations for Independent MPI I/O on the Blue Gene/Q." Parallel Computing 61 (July 2016): 35-51. doi:10.1016/j.parco.2016.07.002.
Marcucci, L. E., G. Mangano, A. Kievsky, and M. Viviani. "Erratum: Implication of the Proton-Deuteron Radiative Capture for Big Bang Nucleosynthesis [Phys. Rev. Lett. 116, 102501 (2016)]." Physical Review Letters 117, no. 4 (July 2016). doi:10.1103/physrevlett.117.049901.

Moulinec, Charles, Juan C. Uribe, Jim Gotts, Bing Xu, and David R. Emerson. "Sleeve Leakage Gas Impact on Fuel Assembly Temperature Distribution." International Journal of Computational Fluid Dynamics 30, no. 6 (July 2016): 419_ 424. doi:10.1080/10618562.2016.1218481.

Perdikaris, Paris, Joseph A. Insley, ${ }^{*}$ Leopold Grinberg, Yue Yu, Michael E. Papka, ${ }^{*}$ and George Em Karniadakis. "Visualizing Multiphysics, Fluid-Structure Interaction Phenomena in Intracranial Aneurysms." Parallel Computing 55 (July 2016): 9-16. doi:10.1016/j.parco.2015.10.016.

Ponga, Mauricio, Amuthan A Ramabathiran, Kaushik Bhattacharya, and Michael Ortiz. "Dynamic Behavior of Nano-Voids in Magnesium Under Hydrostatic Tensile Stress." Modelling and Simulation in Materials Science and Engineering 24, no. 6 (July 2016): 065003. doi:10.1088/0965-0393/24/6/065003.

Reeves, Kyle G., Yi Yao, and Yosuke Kanai. "Electronic Stopping Power in Liquid Water for Protons and $1 \pm$ Particles from First Principles." Physical Review B 94, no. 4 (July 2016). doi:10.1103/physrevb.94.041108.

Shin, Donghyeop, Bayrammurad Saparov, Tong Zhu, William P. Huhn, Volker Blum, and David B. Mitzi. "BaCu $2 \mathrm{Sn}(\mathrm{S}, \mathrm{Se})_{4}$ : Earth-Abundant Chalcogenides for Thin-Film Photovoltaics." Chemistry of Materials 28, no. 13 (July 2016): 4771-4780. doi:10.1021/acs.chemmater.6b01832.

Smith, Cameron W., Brian Granzow, Dan Ibanez, Onkar Sahni, Kenneth E. Jansen, and Mark S. Shephard. "InMemory Integration of Existing Software Components for Parallel Adaptive Unstructured Mesh Workflows." Proceedings of the XSEDE16 on Diversity, Big Data, and Science at Scale - XSEDE16 (July 2016). doi:10.1145/2949550.2949650.

Tu, Qing, Björn Lange, Zehra Parlak, Joao Marcelo J. Lopes, Volker Blum, and Stefan Zauscher. "Quantitative Subsurface Atomic Structure Fingerprint for 2D Materials and Heterostructures by First-Principles-Calibrated ContactResonance Atomic Force Microscopy." ACS Nano 10, no. 7 (July 2016): 6491-6500. doi:10.1021/acsnano.6b02402. 


\section{AUGUST}

Aaboud, M., G. Aad, B. Abbott, J. Abdallah, O. Abdinov, B. Abeloos, R. Aben, ATLAS Collaboration, et al. "Measurement of Exclusive $\gamma y \rightarrow W^{+} W^{-}$Production and Search for Exclusive Higgs Boson Production in $p p$ Collisions At $\sqrt{ } \mathrm{s}=8 \mathrm{TeV}$ using the ATLAS Detector." Physical Review D 94, no. 3 (August 2016). doi:10.1103/physrevd.94.032011.

Aad, G., B. Abbott, J. Abdallah, O. Abdinov, B. Abeloos, R. Aben, O. S. AbouZeid, ATLAS Collaboration, et al. "Measurement of $\mathrm{W} \pm$ and Z-Boson Production Cross Sections in $p p$ Collisions at $\sqrt{ } \mathrm{s}=13 \mathrm{TeV}$ with the ATLAS Detector." Physics Letters B 759 (August 2016): 601-621. doi:10.1016/j.physletb.2016.06.023.

Adhianto, Laksono, and Philip Taffet. "Addressing Challenges in Visualizing Huge Call-Path Traces." 2016 45th International Conference on Parallel Processing Workshops (ICPPW) (August 2016). doi:10.1109/icppw.2016.53.

Ameen, Muhsin M., Xiaofeng Yang, Tang-Wei Kuo, and Sibendu Som. "Parallel Methodology to Capture Cyclic Variability in Motored Engines." International Journal of Engine Research (August 2016): 146808741666254. doi:10.1177/1468087416662544.

Bacmeister, Julio T., Kevin A. Reed, Cecile Hannay, Peter Lawrence, Susan Bates, John E. Truesdale, Nan Rosenbloom, and Michael Levy. "Projected Changes in Tropical Cyclone Activity Under Future Warming Scenarios Using a High-Resolution Climate Model." Climatic Change (August 2016). doi:10.1007/s10584-016-1750-x.

Beckvermit, Jacqueline, Todd Harman, Charles Wight, and Martin Berzins. "Packing Configurations of PBX-9501 Cylinders to Reduce the Probability of a Deflagration to Detonation Transition (DDT)." Propellants, Explosives, Pyrotechnics 41, no. 6 (August 2016): 1070-1078. doi:10.1002/ prep. 201500331.

Bellwied, R. "Sequential Hadronization and the Opportunities It Presents." Journal of Physics: Conference Series 736 (August 2016): 012018. doi:10.1088/17426596/736/1/012018.

Curtis, Farren, Xiaopeng Wang, and Noa Marom. "Effect of Packing Motifs on the Energy Ranking and Electronic Properties of Putative Crystal Structures of Tricyano-

1,4-Dithiino[c]-Isothiazole." Acta Crystallographica Section B Structural Science, Crystal Engineering and Materials 72, no. 4 (August 2016): 562-570. doi:10.1107/ s2052520616009227.
Deshmukh, Sanket A., Lee A. Solomon, Ganesh Kamath, H. Christopher Fry, and Subramanian K. R. S. Sankaranarayanan. "Water Ordering Controls the Dynamic Equilibrium of Micelle-Fibre Formation in Self-Assembly of Peptide Amphiphiles." Nature Communications 7 (August 2016): 12367. doi:10.1038/ncomms12367.

Erdemir, Ali, Giovanni Ramirez, Osman L. Eryilmaz, Badri Narayanan, Yifeng Liao, Ganesh Kamath, and Subramanian K. R. S. Sankaranarayanan. "Carbon-Based Tribofilms from Lubricating Oils.” Nature 536, no. 7614 (August 2016): 67-71. doi:10.1038/nature18948.

Jiang, Yan-Fei, Shane W. Davis, and James M. Stone. "Iron Opacity Bump Changes the Stability and Structure of Accretion Disks in Active Galactic Nuclei." The Astrophysical Journal 827, no. 1 (August 2016): 10. doi:10.3847/0004$637 \times / 827 / 1 / 10$.

Jo, Sunhwan, Donghyuk Suh, Ziwei He, Christophe Chipot, and Benoît Roux. "Leveraging the Information from Markov State Models To Improve the Convergence of Umbrella Sampling Simulations." The Journal of Physical Chemistry B 120, no. 33 (August 2016): 8733-8742. doi:10.1021/acs. jpcb.6b05125.

Li, Nan, Michael D. Gladders, Esteban M. Rangel, Michael K. Florian, Lindsey E. Bleem, Katrin Heitmann, Salman Habib, and Patricia Fasel. "PICS: Simulations of Strong Gravitational Lensing In Galaxy Clusters.” The Astrophysical Journal 828, no. 1 (August 2016): 54. doi:10.3847/0004-637x/828/1/54.

Liu, Miao, Anubhav Jain, Ziqin Rong, Xiaohui Qu, Pieremanuele Canepa, Rahul Malik, Gerbrand Ceder, and Kristin A. Persson. "Evaluation of Sulfur Spinel Compounds for Multivalent Battery Cathode Applications." Energy Environ. Sci. 9, no. 10 (August 2016): 3201-3209. doi:10.1039/ c6ee01731b.

Lovato, A., S. Gandolfi, J. Carlson, Steven C. Pieper, and R. Schiavilla. "Electromagnetic Response of C12: A FirstPrinciples Calculation.” Physical Review Letters 117, no. 8 (August 2016). doi:10.1103/physrevlett.117.082501.

Meng, Jintao, Sangmin Seo, Pavan Balaji, Yanjie Wei, Bingqiang Wang, and Shenzhong Feng. "SWAP-Assembler 2: Optimization of De Novo Genome Assembler at Extreme Scale." 2016 45th International Conference on Parallel Processing (ICPP) (August 2016). doi:10.1109/icpp.2016.29.

Ratti, Claudia. "Recent Results on Lattice QCD Thermodynamics." Journal of Physics: Conference Series 736 (August 2016): 012001. doi:10.1088/17426596/736/1/012001. 


\section{ALCF PUBLICATIONS CONTINUED}

Ray, Navamita, Iulian Grindeanu, Xinglin Zhao, Vijay Mahadevan, and Xiangmin Jiao. "Array-Based, Parallel Hierarchical Mesh Refinement Algorithms for Unstructured Meshes." Computer-Aided Design (August 2016). doi:10.1016/j.cad.2016.07.011.

Reilly, Anthony M., Richard I. Cooper, Claire S. Adjiman, Saswata Bhattacharya, A. Daniel Boese, Jan Gerit Brandenburg, Peter J. Bygrave, et al. "Report on the Sixth Blind Test of Organic Crystal Structure Prediction Methods." Acta Crystallographica Section B Structural Science, Crystal Engineering and Materials 72, no. 4 (August 2016): 439-459. doi:10.1107/s2052520616007447.

Scheel, Janet D., and Jörg Schumacher. "Global and Local Statistics in Turbulent Convection at Low Prandtl Numbers." Journal of Fluid Mechanics 802 (August 2016): 147-173. doi:10.1017/jfm.2016.457.

Scherpelz, Peter, Marco Govoni, Ikutaro Hamada, and Giulia Galli. "Implementation and Validation of Fully Relativistic GW Calculations: Spin-Orbit Coupling in Molecules, Nanocrystals, and Solids." Journal of Chemical Theory and Computation 12, no. 8 (August 2016): 3523-3544. doi:10.1021/acs.jctc.6b00114.

\section{SEPTEMBER}

Aaboud, M., G. Aad, B. Abbott, J. Abdallah, O. Abdinov, B. Abeloos, ATLAS Collaboration, et al. "Measurement of Jet Activity in Top Quark Events Using the e $\mu$ Final State with Two b-Tagged Jets in $p p$ Collisions at $\sqrt{ } s=8 \mathrm{TeV}$ with the ATLAS Detector." Journal of High Energy Physics 2016, no. 9 (September 2016). doi:10.1007/jhep09(2016)074.

Agrawal, B. R., and A. Sharma. "Numerical Analysis of Aerodynamic Noise Mitigation via Leading Edge Serrations for a Rod-Airfoil Configuration." International Journal of Aeroacoustics 15, no. 8 (September 2016): 734-756. doi:10.1177/1475472×16672322.

Bhardwaj, Gaurav, Vikram Khipple Mulligan, Christopher D. Bahl, Jason M. Gilmore, Peta J. Harvey, Olivier Cheneval, Garry W. Buchko, Surya V. S. R. K. Pulavarti, Quentin Kaas, Alexander Eletsky, Po-Ssu Huang, William A. Johnsen, Per Jr Greisen, Gabriel J. Rocklin, Yifan Song, Thomas W. Linsky, Andrew Watkins, Stephen A. Rettie, Xianzhong Xu, Lauren P. Carter, Richard Bonneau, James M. Olson, Evangelos Coutsias, Colin E. Correnti, Thomas Szyperski, David J. Craik, David Baker. "Accurate de Novo Design of Hyperstable Constrained Peptides." Nature 538, no. 7625 (September 2016): 329-335. doi:10.1038/nature19791.
Boughezal, Radja, Xiaohui Liu, and Frank Petriello. "A Comparison of NNLO QCD Predictions with $7 \mathrm{TeV}$ ATLAS and CMS Data for V+jet Processes." Physics Letters B 760 (September 2016): 6-13. doi:10.1016/j.physletb.2016.06.032.

Chien, A., P. Balaji, N. Dun, A. Fang, H. Fujita, K. Iskra, Z. Rubenstein, Z. Zheng, J. Hammond, I. Laguna, D. Richards, A. Dubey, B. van Straalen, M. Hoemmen, M. Heroux, K. Teranishi, and A. Siegel. "Exploring Versioned Distributed Arrays for Resilience in Scientific Applications: Global View Resilience." International Journal of High Performance Computing Applications (September 2016). doi:10.1177/1094342016664796.

Elenewski, Justin E., Jesse Y. Cai, Wei Jiang, and Hanning Chen. "Functional Mode Hot Electron Transfer Theory." The Journal of Physical Chemistry C 120, no. 37 (September 2016): 20579-20587. doi:10.1021/acs. jpcc.6b00099.

Forsberg, U., D. Rudolph, L.-L. Andersson, A. Di Nitto, Ch.E. Düllmann, C. Fahlander, J.M. Gates, J. M. Gates, P. Golubev, K. E. Gregorich, C. J. Gross, R.-D. Herzberg, F. P. Heßberger, J. Khuyagbaatar, J. V. Kratz, K. Rykaczewski, L. G. Sarmiento, M. Schädel, A. Yakushev, S. Åberg, D. Ackermann, M. Block, H. Brand, B. G. Carlsson, D. Cox, X. Derkx, J. Dobaczewski, K. Eberhardt, J. Even, J. Gerl, E. Jäger, B. Kindler, J. Krier, I. Kojouharov, N. Kurz, B. Lommel, A. Mistry, C. Mokry, W. Nazarewicz, H. Nitsche, J. P. Omtvedt, P. Papadakis, I. Ragnarsson, J. Runke, H. Schaffner, B. Schausten, Yue Shi, P. Thörle-Pospiech, T. Torres, T. Traut, N. Trautmann, A. Türler, A. Ward, D. E. Ward, and N. Wiehl. "Recoil-a-Fission and Recoil-a-a-Fission Events Observed in the Reaction 48Ca + 243Am." Nuclear Physics A 953 (September 2016): 117-138. doi:10.1016/j.nuclphysa.2016.04.025.

Goldey, Matthew B., Daniel Reid, Juan de Pablo, and Giulia Galli. "Planarity and Multiple Components Promote Organic Photovoltaic Efficiency by Improving Electronic Transport." Phys. Chem. Chem. Phys. 18, no. 46 (September 2016): 31388-31399. doi:10.1039/c6cp04999k.

King, J. R., K. H. Burrell, A. M. Garofalo, R. J. Groebner, S. E. Kruger, A. Y. Pankin, and P. B. Snyder. "NIMROD Modeling of Quiescent H-Mode: Reconstruction Considerations and Saturation Mechanism." Nuclear Fusion 57, no. 2 (September 2016): 022002. doi:10.1088/0029$5515 / 57 / 2 / 022002$. 
Li, Xiao, Julio C. Armas-Perez, Jose A. Martinez-Gonzalez, Xiaoying Liu, Helou Xie, Camille Bishop, Juan P. HernandezOrtiz, Rui Zhang, Juan J. de Pablo, and Paul F. Nealey. "Directed Self-Assembly of Nematic Liquid Crystals on Chemically Patterned Surfaces: Morphological States and Transitions." Soft Matter 12, no. 41 (September 2016): 85958605. doi:10.1039/c6sm01733a.

Miorelli, M., S. Bacca, N. Barnea, G. Hagen, G. R. Jansen, G. Orlandini, and T. Papenbrock. "Electric Dipole Polarizability from First Principles Calculations." Physical Review C 94, no. 3 (September 2016). doi:10.1103/physrevc.94.034317.

Palacio-Betancur, Viviana, Stiven Villada-Gil, Juan J. de Pablo, and Juan P. Hernández-Ortiz. "Educating Local Radial Basis Functions Using the Highest Gradient of Interest in Three Dimensional Geometries." International Journal for Numerical Methods in Engineering (September 2016). doi:10.1002/nme.5368.

Rangel, Esteban, Nan Li, Salman Habib, Tom Peterka, Ankit Agrawal, Wei-Keng Liao, and Alok Choudhary. "Parallel DTFE Surface Density Field Reconstruction.” 2016 IEEE International Conference on Cluster Computing (CLUSTER) (September 2016). doi:10.1109/cluster.2016.40.

Shin, Donghyeop, Bayrammurad Saparov, Tong Zhu, William P. Huhn, Volker Blum, and David B. Mitzi. "Chemlnform Abstract: BaCu2Sn(S,Se)4: Earth-Abundant Chalcogenides for Thin-Film Photovoltaics." ChemInform 47, no. 39 (September 2016). doi:10.1002/chin.201639006.

Wallace, Sean, Zhou Zhou, Venkatram Vishwanath,* Susan Coghlan,* John Tramm, Zhiling Lan, and Michael E. Papka.* "Application Power Profiling on IBM Blue Gene/Q." Parallel Computing 57 (September 2016): 73-86. doi:10.1016/j. parco.2016.05.015.

Wolf, Laura.* "Multiyear Simulation Study Provides Breakthrough in Membrane Protein Research." Computing in Science \& Engineering 18, no. 5 (September 2016): 94-97. doi:10.1109/mcse.2016.95.

Zheng, X., and G. E. Karniadakis. "A Phase-field/ALE Method for Simulating Fluid-Structure Interactions in Two-Phase Flow." Computer Methods in Applied Mechanics and Engineering 309 (September 2016): 19-40. doi:10.1016/j. cma.2016.04.035.

Zhu, Wenlu, Florian Fusseis, Harrison Lisabeth, Tiange Xing, Xianghui Xiao, Vincent De Andrade, and Shun-ichiro Karato. "Experimental Evidence of Reaction-Induced Fracturing During Olivine Carbonation.” Geophysical Research Letters 43, no. 18 (September 2016): 9535-9543. doi:10.1002/2016gl070834.

\section{OCTOBER}

Aaboud, M., G. Aad, B. Abbott, J. Abdallah, O. Abdinov, B. Abeloos, R. Aben, ATLAS Collaboration, et al. "Measurement of the Top Quark Mass in the $\mathrm{tt}^{-} \rightarrow$ Dilepton Channel from $\sqrt{ } \mathrm{s}=8 \mathrm{TeV}$ ATLAS Data." Physics Letters B 761 (October 2016): 350-371. doi:10.1016/j.physletb.2016.08.042.

Arkani-Hamed, Nima, Tao Han, Michelangelo Mangano, and Lian-Tao Wang. "Physics Opportunities of a 100 TeV ProtonProton Collider." Physics Reports 652 (October 2016): 1-49. doi:10.1016/j.physrep.2016.07.004.

Boughezal, Radja, Xiaohui Liu, and Frank Petriello. "Phenomenology of the Z-boson Plus Jet Process at NNLO." Physical Review D 94, no. 7 (October 2016). doi:10.1103/ physrevd.94.074015.

Brawand, Nicholas P., Márton Vörös, Marco Govoni, and Giulia Galli. "Generalization of Dielectric-Dependent Hybrid Functionals to Finite Systems." Physical Review X 6, no. 4 (October 2016). doi:10.1103/physrevx.6.041002.

Chang, Hung-Yu, Xuejin Li, He Li, and George Em Karniadakis. "MD/DPD Multiscale Framework for Predicting Morphology and Stresses of Red Blood Cells in Health and Disease." Edited by Andrew D. McCulloch. PLOS Computational Biology 12, no. 10 (October 2016): e1005173. doi:10.1371/journal.pcbi.1005173.

Chung, Y. G., D. A. Gomez-Gualdron, P. Li, K. T. Leperi, P. Deria, H. Zhang, N. A. Vermeulen, J. F. Stoddart, F. You, J. T. Hupp, O. K. Farha, R. Q. Snurr. "In Silico Discovery of Metal-Organic Frameworks for Precombustion CO2 Capture Using a Genetic Algorithm." Science Advances 2, no. 10 (October 2016): e1600909-e1600909. doi:10.1126/ sciadv.1600909.

Deng, Mingge, Zhen Li, Oleg Borodin, and George Em Karniadakis. "cDPD: A New Dissipative Particle Dynamics Method for Modeling Electrokinetic Phenomena at the Mesoscale." The Journal of Chemical Physics 145, no. 14 (October 2016): 144109. doi:10.1063/1.4964628.

Fajer, Mikolai, Yilin Meng, and Benoît Roux. "The Activation of c-Src Tyrosine Kinase: Conformational Transition Pathway and Free Energy Landscape." The Journal of Physical Chemistry B (October 2016). doi:10.1021/acs.jpcb.6b08409.

Featherstone, Nicholas A., and Bradley W. Hindman. "The Emergence of Solar Supergranulation as a Natural Consequence of Rotationally Constrained Interior Convection." The Astrophysical Journal 830, no. 1 (October 2016): L15. doi:10.3847/2041-8205/830/1/115. 


\section{ALCF PUBLICATIONS CONTINUED}

Hagen, G., G. R. Jansen, and T. Papenbrock. "Structure of Ni78 from First-Principles Computations." Physical Review Letters 117, no. 17 (October 2016). doi:10.1103/ physrevlett.117.172501.

Li, C. K., P. Tzeferacos, D. Lamb, G. Gregori, P. A. Norreys, M. J. Rosenberg, R. K. Follett, D. H. Froula, M. Koenig, F. H. Seguin, J. A. Frenje, H. G. Rinderknecht, H. Sio, A. B. Zylstra, R. D. Petrasso, P. A. Amendt, H. S. Park, B. A. Remington, D. D. Ryutov, S. C. Wilks, R. Betti, A. Frank, S. X. Hu, T. C. Sangster, P. Hartigan, R. P. Drake, C. C. Kuranz, S. V. Lebedev, and N. C. Woolsey. "Scaled Laboratory Experiments Explain the Kink Behaviour of the Crab Nebula Jet." Nature Communications 7 (October 2016): 13081. doi:10.1038/ncomms13081.

Pan, D., and G. Galli. "The Fate of Carbon Dioxide in WaterRich Fluids under Extreme Conditions." Science Advances 2, no. 10 (October 2016): e1601278-e1601278. doi:10.1126/ sciadv.1601278.

Ponga, M., K. Bhattacharya, and M. Ortiz. "A SublinearScaling Approach to Density-Functional-Theory Analysis of Crystal Defects." Journal of the Mechanics and Physics of Solids 95 (October 2016): 530-556. doi:10.1016/j. jmps.2016.05.029.

Radak, Brian K.," and Benoît Roux. "Efficiency in Nonequilibrium Molecular Dynamics Monte Carlo Simulations." The Journal of Chemical Physics 145, no. 13 (October 2016): 134109. doi:10.1063/1.4964288.

Read, Sarah, and Michael E. Papka.* "Improving Models of Document Cycling: Accounting for the Less Visible Writing Activities of an Annual Reporting Process at a Supercomputing Facility." 2016 IEEE International Professional Communication Conference (IPCC) (October 2016). doi:10.1109/ipcc.2016.7740504.

Schunck, N., and L. M. Robledo. "Microscopic Theory of Nuclear Fission: A Review." Reports on Progress in Physics 79, no. 11 (October 2016): 116301. doi:10.1088/00344885/79/11/116301.

Sullivan, Raymond, Junteng Jia, Álvaro Vázquez-Mayagoitia,* and Antonio Picón. "Normal Auger Processes with Ultrashort X-Ray Pulses in Neon." Physical Review A 94, no. 4 (October 2016). doi:10.1103/physreva.94.043421.

Wu, Jingjin, Xuanxing Xiong, Eduardo Berrocal, Jia Wang, and Zhiling Lan. "Topology Mapping of Irregular Parallel Applications on Torus-Connected Supercomputers." The Journal of Supercomputing (October 2016). doi:10.1007/ s11227-016-1876-7.
Zhao, Dongfang, Ke Wang, Kan Qiao, Tonglin Li, Iman Sadooghi, and Ioan Raicu. "Toward High-Performance KeyValue Stores through GPU Encoding and Locality-Aware Encoding." Journal of Parallel and Distributed Computing 96 (October 2016): 27-37. doi:10.1016/j.jpdc.2016.04.015.

Zhou, Zhou, Xu Yang, Dongfang Zhao, Paul Rich,, Wei Tang, Jia Wang, and Zhiling Lan. "I/O-Aware Bandwidth Allocation for Petascale Computing Systems." Parallel Computing 58 (October 2016): 107-116. doi:10.1016/j.parco.2016.05.005.

\section{NOVEMBER}

Aaboud, M., G. Aad, B. Abbott, J. Abdallah, O. Abdinov, B. Abeloos, R. Aben, ATLAS Collaboration, et al. "Measurement of Top Quark Pair Differential Cross Sections in the Dilepton Channel in $p p$ Collisions at $\sqrt{ } \mathrm{s}=7$ and $8 \mathrm{TeV}$ with ATLAS." Physical Review D 94, no. 9 (November 2016). doi:10.1103/ physrevd.94.092003.

Azmandian, Mahdi, Mark Hancock, Hrvoje Benko, Eyal Ofek, and Andrew D. Wilson. "A Demonstration of Haptic Retargeting." Proceedings of the 2016 ACM on Interactive Surfaces and Spaces - ISS "16 (November 2016). doi:10.1145/2992154.2996883.

Brown, C. S., and I. A. Bolotnov. "Spectral Analysis of Single- and Two-Phase Bubbly DNS in Different Geometries." Nuclear Science and Engineering 184, no. 3 (November 2016). doi:10.13182/nse15-126.

Cunningham, Greg, and Katie Elyce Jones. "Argonne Discovery Yields Self-Healing Diamond-Like Carbon." Computing in Science \& Engineering 18, no. 6 (November 2016): 77-79. doi:10.1109/mcse.2016.102.

Luo, Ye, Anouar Benali," Luke Shulenburger, Jaron T. Krogel, Olle Heinonen, and Paul R. C. Kent. "Phase Stability of TiO2 Polymorphs from Diffusion Quantum Monte Carlo." New Journal of Physics 18, no. 11 (November 2016): 113049. doi:10.1088/1367-2630/18/11/113049.

Marrinan, Thomas, Arthur Nishimoto, Joseph A. Insley,* Silvio Rizzi,* Andrew Johnson, and Michael E. Papka.* "Interactive Multi-Modal Display Spaces for Visual Analysis." Proceedings of the 2016 ACM on Interactive Surfaces and Spaces - ISS "16 (November 2016). doi:10.1145/2992154.2996792. 
Merzari, Elia, Aleks Obabko, Paul Fischer, Noah Halford, Justin Walker, Andrew Siegel, and Yiqi Yu. "Large-Scale Large Eddy Simulation of Nuclear Reactor Flows: Issues and Perspectives." Nuclear Engineering and Design (November 2016). doi:10.1016/j.nucengdes.2016.09.028.

Nazarian, Dalar, Jeffrey S. Camp, Yongchul G. Chung, Randall Q. Snurr, and David S. Sholl. "Large-Scale Refinement of Metal-Organic Framework Structures Using Density Functional Theory." Chemistry of Materials (November 2016). doi:10.1021/acs.chemmater.6b04226.

Perez-Monte, Cristian F., Mauricio D. Perez, Silvio Rizzi,* Fabiana Piccoli, and Cristian Luciano. "Modelling Frame Losses in a Parallel Alternate Frame Rendering System with a Computational Best-Effort Scheme." Computers \& Graphics 60 (November 2016): 76-82. doi:10.1016/j. cag.2016.08.004.

Reddy, Sandeep K., Shelby C. Straight, Pushp Bajaj, C. Huy Pham, Marc Riera, Daniel R. Moberg, Miguel A. Morales, Chris Knight," Andreas W. Götz, and Francesco Paesani. "On the Accuracy of the MB-Pol Many-Body Potential for Water: Interaction Energies, Vibrational Frequencies, and Classical Thermodynamic and Dynamical Properties from Clusters to Liquid Water and Ice." The Journal of Chemical Physics 145, no. 19 (November 2016): 194504. doi:10.1063/1.4967719.

Romero-Redondo, Carolina, Sofia Quaglioni, Petr Navrátil, and Guillaume Hupin. "How Many-Body Correlations and a Clustering Shape He6." Physical Review Letters 117, no. 22 (November 2016). doi:10.1103/physrevlett.117.222501.

Tessier, Francois," Preeti Malakar,' Venkatram Vishwanath," Emmanuel Jeannot, and Florin Isaila. "Topology-Aware Data Aggregation for Intensive I/O on Large-Scale Supercomputers." 2016 First International Workshop on Communication Optimizations in HPC (COMHPC) (November 2016). doi:10.1109/comhpc.2016.013.

Zawislak, Dawid, ${ }^{*}$ Brian Toonen," William Allcock, Silvio Rizzi, Joseph A. Insley,* Venkatram Vishwanath,* and Michael E. Papka.* "Early Investigations into Using a Remote RAM Pool with the VI3 Visualization Framework." 2016 Second Workshop on In Situ Infrastructures for Enabling Extreme-Scale Analysis and Visualization (ISAV) (November 2016). doi:10.1109/isav.2016.010.

Zen, Andrea, Loïc M. Roch, Stephen J. Cox, Xiao Liang Hu, Sandro Sorella, Dario Alfè, and Angelos Michaelides. "Toward Accurate Adsorption Energetics on Clay Surfaces." The Journal of Physical Chemistry C 120, no. 46 (November 2016): 26402-26413. doi:10.1021/acs. jpcc.6b09559.
Zhou, Zhou, Xu Yang, Zhiling Lan, Paul Rich,* Wei Tang, Vitali Morozov," and Narayan Desai. "Improving Batch Scheduling on Blue Gene/Q by Relaxing Network Allocation Constraints." IEEE Transactions on Parallel and Distributed Systems 27, no. 11 (November 2016): 3269-3282. doi:10.1109/ tpds.2016.2528247.

\section{DECEMBER}

Aaboud, M., G. Aad, B. Abbott, J. Abdallah, O. Abdinov, B. Abeloos, R. Aben, ATLAS Collaboration, et al. "Measurement of W+W- Production in Association with One Jet in Proton-Proton Collisions at $\sqrt{ } \mathrm{s}=8 \mathrm{TeV}$ with the ATLAS Detector." Physics Letters B 763 (December 2016): 114-133. doi:10.1016/j.physletb.2016.10.014.

Abelof, Gabriel, Radja Boughezal, Xiaohui Liu, and Frank Petriello. "Single-Inclusive Jet Production in ElectronNucleon Collisions through Next-to-Next-to-Leading Order in Perturbative QCD." Physics Letters B 763 (December 2016): 52-59. doi:10.1016/j.physletb.2016.10.022.

Boughezal, Radja, Xiaohui Liu, and Frank Petriello. "W-Boson Plus Jet Differential Distributions at NNLO in QCD." Physical Review D 94, no. 11 (December 2016). doi:10.1103/ physrevd.94.113009.

Calci, Angelo, Petr Navrátil, Robert Roth, Jérémy DohetEraly, Sofia Quaglioni, and Guillaume Hupin. "Can Ab Initio Theory Explain the Phenomenon of Parity Inversion in Be11?" Physical Review Letters 117, no. 24 (December 2016). doi:10.1103/physrevlett.117.242501.

Christ, Norman H., Xu Feng, Andreas Jüttner, Andrew Lawson, Antonin Portelli, and Christopher T. Sachrajda. "First Exploratory Calculation of the Long-Distance Contributions to the Rare Kaon Decays $K \rightarrow \Pi P+P$-." Physical Review D 94 , no. 11 (December 2016). doi:10.1103/physrevd.94.114516.

Churchill, R. M., J. M. Canik, C. S. Chang, R. Hager, A. W. Leonard, R. Maingi, R. Nazikian, and D. P. Stotler. "Kinetic Simulations of Scrape-Off Layer Physics in the DIII-D Tokamak." Nuclear Materials and Energy (December 2016). doi:10.1016/j.nme.2016.12.013.

García-Hernández, Erwin, Roberto Flores-Moreno, Álvaro Vázquez-Mayagoitia*, Rubicelia Vargas, and Jorge Garza. "Initial Stage of the Degradation of Three Common Neonicotinoids: Theoretical Prediction of Charge Transfer Sites." New J. Chem. 41, no. 3 (December 2016): 965-974. doi:10.1039/c6nj02655a. 


\section{ALCF PUBLICATIONS CONTINUED}

Gnedin, Nickolay Y. "On the Proper Use of the Reduced Speed of Light Approximation." The Astrophysical Journal 833, no. 1 (December 2016): 66. doi:10.3847/15384357/833/1/66.

Ho, Phay J., Chris Knight," Miklos Tegze, Gyula Faigel, C. Bostedt, and L. Young. "Atomistic Three-Dimensional Coherent X-Ray Imaging of Nonbiological Systems." Physical Review A 94, no. 6 (December 2016). doi:10.1103/ physreva.94.063823.

Keefer, Derek W., Huiyang Gou, Andrew P. Purdy, Albert Epshteyn, Duck Young Kim, John V. Badding, and Timothy A. Strobel. "Pressure-Induced Polymerization of LiN(CN)2." The Journal of Physical Chemistry A 120, no. 47 (December 2016): 9370-9377. doi:10.1021/acs.jpca.6b06780.

Lovato, A. “Green's Function Monte Carlo Calculations of the Electromagnetic and Neutral-Weak Response Functions in the Quasi-Elastic Sector." Proceedings of the 10th International Workshop on Neutrino-Nucleus Interactions in Few-GeV Region (Nulnt15) (December 2016). doi:10.7566/ jpscp.12.010031.

Marin, O., R. Vinuesa, A. V. Obabko, and P. Schlatter. "Characterization of the Secondary Flow in Hexagonal Ducts." Physics of Fluids 28, no. 12 (December 2016): 125101. doi:10.1063/1.4968844.

Murphy, John T., Elif Seyma Bayrak, Mustafa Cagdas Ozturk, and Ali Cinar. "Simulating 3-D Bone Tissue Growth Using Repast HPC: Initial Simulation Design and Performance Results." 2016 Winter Simulation Conference (WSC) (December 2016). doi:10.1109/wsc.2016.7822252.

Ozik, Jonathan, Nicholson T. Collier, Justin M. Wozniak, and Carmine Spagnuolo. "From Desktop to Large-Scale Model Exploration with Swift/T." 2016 Winter Simulation Conference (WSC) (December 2016). doi:10.1109/wsc.2016.7822090.

Park, Hahnbeom, Philip Bradley, Per Greisen, Yuan Liu, Vikram Khipple Mulligan, David E. Kim, David Baker, and Frank DiMaio. "Simultaneous Optimization of Biomolecular Energy Functions on Features from Small Molecules and Macromolecules." Journal of Chemical Theory and Computation 12, no. 12 (December 2016): 6201-6212. doi:10.1021/acs.jctc.6b00819.
Peng, Chong, Justus A. Calvin, Fabijan Pavošević, Jinmei Zhang, and Edward F. Valeev. "Massively Parallel Implementation of Explicitly Correlated Coupled-Cluster Singles and Doubles Using TiledArray Framework." The Journal of Physical Chemistry A 120, no. 51 (December 2016): 10231-10244. doi:10.1021/acs.jpca.6b10150.

Petra, Cosmin G., Victor M. Zavala, Elias D. Nino-Ruiz, and Mihai Anitescu. "A High-Performance Computing Framework for Analyzing the Economic Impacts of Wind Correlation." Electric Power Systems Research 141 (December 2016): 372-380. doi:10.1016/j.epsr.2016.08.010.

Ratti, Claudia. "Lattice QCD: Bulk and Transport Properties of QCD Matter." Nuclear Physics A 956 (December 2016): 51-58. doi:10.1016/j.nuclphysa.2016.02.022.

Rocco, Noemi, Alessandro Lovato, and Omar Benhar. "Comparison of the Electromagnetic Responses of C12 Obtained from the Green's Function Monte Carlo and Spectral Function Approaches." Physical Review C 94, no. 6 (December 2016). doi:10.1103/physrevc.94.065501.

Schumacher, Jörg, Vinodh Bandaru, Ambrish Pandey, and Janet D. Scheel. "Transitional Boundary Layers in LowPrandtl-Number Convection." Physical Review Fluids 1, no. 8 (December 2016). doi:10.1103/physrevfluids.1.084402.

See, Kimberly A., Heng-Liang Wu, Kah Chun Lau, Minjeong Shin, Lei Cheng, Mahalingam Balasubramanian, Kevin G. Gallagher, Larry A. Curtiss, and Andrew A. Gewirth. "Effect of Hydrofluoroether Cosolvent Addition on Li Solvation in Acetonitrile-Based Solvate Electrolytes and Its Influence on S Reduction in a Li-S Battery." ACS Applied Materials \& Interfaces 8, no. 50 (December 2016): 34360-34371. doi:10.1021/acsami.6b11358.

Usher, Will, Ingo Wald, Aaron Knoll, Michael E. Papka, ${ }^{*}$ and Valerio Pascucci, "In Situ Exploration of Particle Simulations with CPU Ray Tracing." Supercomputing Frontiers and Innovations 3, no. 4 (December 2016). doi:10.14529/ jsfi160401.

Zheng, Qinmin, David P. Durkin, Justin E. Elenewski, Yingxue Sun, Nathan A. Banek, Likun Hua, Hanning Chen, Michael J. Wagner, Wen Zhang, and Danmeng Shuai. "Visible-Light-Responsive Graphitic Carbon Nitride: Rational Design and Photocatalytic Applications for Water Treatment." Environmental Science \& Technology 50, no. 23 (December 2016): 12938-12948. doi:10.1021/acs. est.6b02579. 
APPENDIX II: ALCF PROJECTS 


\section{INCITE PROJECTS}

\section{BIOLOGICAL SCIENCES}

Multiscale Simulations of Human Pathologies

George Karniadakis, Brown University

90 Million Core-Hours (ALCF: 50M;

OLCF: 40M)

Protein-Protein Binding Specificity

Benoît Roux, The University of

Chicago/Argonne National Laboratory

160 Million Core-Hours

\section{CHEMISTRY}

Evaluation of a 1000 MW Commercial Ultra Super-Critical Coal Boiler

Martin Berzins, University of Utah 351 Million Core-Hours (ALCF: 280M;

OLCF: 71M)

First-Principles Simulations of High-Speed Combustion and Detonation

Alexei Khokhlov, The University of Chicago

140 Million Core-Hours

Towards Breakthroughs in Protein Structure Calculation and Design David Baker, University of Washington 120 Million Core-Hours

\section{COMPUTER SCIENCE}

Dynamic and Adaptive Parallel Programming for Exascale Research Robert Harrison, Stony Brook

University

20 Million Core-Hours

Performance Evaluation and Analysis Consortium (PEAC) End Station Leonid Oliker, Lawrence Berkeley National Laboratory 90 Million Core-Hours (ALCF: 45M; OLCF: 45M)

\section{EARTH SCIENCE}

Accelerated Climate Modeling for Energy

Mark Taylor, Sandia National

Laboratories

180 Million Core-Hours (ALCF: 100M;

OLCF: 80M)

Frontiers in Planetary and Stellar

Magnetism Through

High-Performance Computing

Jonathan Aurnou, University of

California, Los Angeles

150 Million Core-Hours

High-Frequency Ground Motion

Simulation for Seismic Hazard

Analysis

Thomas Jordan, University of Southern

California

190 Million Core-Hours (ALCF: 90M;

OLCF: 100M)

\section{ENGINEERING}

Adaptive Detached-Eddy Simulation of a High Lift Wing with Active Flow Control

Kenneth Jansen, University of

Colorado Boulder

50 Million Core-Hours

Convective Turbulence in Liquid

Gallium and Sodium

Janet Scheel, Occidental College

80 Million Core-Hours

Direct Numerical Simulation of

Compressible, Turbulent Flow

Jonathan Poggie, Purdue University

150 Million Core-Hours

Direct Numerical Simulations and Robust Predictions of Cloud Cavitation Collapse

Petros Koumoutsakos, ETH Zürich

72 Million Core-Hours

Large-Eddy Simulations of Combustor Liner Flows Anne Dord, GE Aviation 100 Million Core-Hours

\section{MATERIALS SCIENCE}

Charge Transport in Thin Film Ionomers

Gregory Voth, The University of

Chicago

100 Million Core-Hours

Combining High-Accuracy Electronic Structure Methods to Study Surface Reactions

Maria Chan, Argonne National

Laboratory

50 Million Core-Hours

Computational Spectroscopy of Heterogeneous Interfaces

Giulia Galli, The University of Chicago/ Argonne National Laboratory

François Gygi, University of California, Davis

150 Million Core-Hours

Electronic Response to Particle Radiation in Condensed Matter André Schleife, University of Illinois at Urbana-Champaign

70 Million Core-Hours

Petascale Simulations of

Self-Healing Nanomaterials

Rajiv Kalia, University of Southern

California

180 Million Core-Hours

QMC Simulations Database for Predictive Modeling and Theory David Ceperley, University of Illinois at Urbana-Champaign 170 Million Core-Hours (ALCF: 90M; OLCF: 80M)

Reactive MD Simulations of Electrochemical Oxide Interfaces at Mesoscale

Subramanian Sankaranarayanan, Argonne National Laboratory 40 Million Core-Hours

SiO2 Fracture: Chemomechanics with a Machine Learning Hybrid QM/MM Scheme

James Kermode, University of Warwick 126 Million Core-Hours 
State-of-the-Art Simulations of Liquid Phenomena

Mark Gordon, Iowa State University

200 Million Core-Hours

Unveiling the Behavior of UO2 Under Extreme Physical Conditions

Peter Littlewood, Argonne National

Laboratory

75 Million Core-Hours

\section{PHYSICS}

Cosmic Reionization on Computers

Nickolay Gnedin, Fermilab

65 Million Core-Hours

Cosmological Simulations for Large-Scale Sky Surveys

Salman Habib, Argonne National

Laboratory

150 Million Core-Hours (ALCF: 80M;

OLCF: 7OM)

Fundamental Properties of QCD Matter Produced at RHIC and the LHC

Claudia Ratti, University of Houston

100 Million Core-Hours

Kinetic Simulations of Relativistic Radiative Magnetic Reconnection Dmitri Uzdensky, University of

Colorado Boulder

90 Million Core-Hours

\section{Lattice QCD}

Paul Mackenzie, Fermilab

280 Million Core-Hours (ALCF: 180M; OLCF: 100M)

Magnetohydrodynamic Models of Accretion, Including Radiation Transport James Stone, Princeton University 47 Million Core-Hours

Nuclear Structure and Nuclear Reactions James Vary, lowa State University 184 Million Core-Hours (ALCF: 80M; OLCF: 104M)
Petascale Simulation of Magnetorotational Core-Collapse Supernovae

Sean Couch, Michigan State University 100 Million Core-Hours

Petascale Simulations of Laser Plasma Interaction Relevant to IFE Frank Tsung, University of California, Los Angeles 100 Million Core-Hours

\section{5-2016 ALCC PROJECTS}

\section{CHEMISTRY}

Anomalous Density Properties and Ion Solvation in Liquid Water: A PathIntegral Ab Initio Study

Robert A. DiStasio, Cornell University

175 Million Core-Hours

Computational Design of Interfaces for Photovoltaics

Noa Marom, Tulane University 120 Million Core-Hours (ALCF: 100M; NERSC: 20M)

PT-Symmetric Quantum Mechanics for Real-Time Electron Transport Simulations

Hanning Chen, George Washington

University

16 Million Core-Hours

\section{COMPUTER SCIENCE}

Demonstration of the Scalability of Programming Environments by Simulating Multiscale Applications Robert Voigt, Leidos Inc.

167 Million Core-Hours

(ALCF: 127M; OLCF: 4OM)

Performance Analysis, Modeling, and Scaling of HPC Applications and Tools

Abhinav Bhatele, Lawrence Livermore National Laboratory

29.4 Million Core-Hours (ALCF: 20.1M; OLCF: 9.3M)
Portable Application Development for Next-Generation Supercomputer Architectures

Tjerk Straatsma, Oak Ridge National Laboratory

160 Million Core-Hours (ALCF: 60M;

NERSC: 4OM; OLCF: 6OM)

\section{EARTH SCIENCE}

Delivering the Department of Energy's Next-Generation High-Resolution Earth System Model Peter Thornton, Oak Ridge National

Laboratory

165 Million Core-Hours (ALCF: 110M; OLCF: 55M)

Validation of RAP/HRRR for the Wind Forecast Improvement Project II Joe Olson, National Oceanic and Atmospheric Administration 15 Million Core-Hours

\section{ENGINEERING}

Advancing Internal Combustion Engine Simulations Using Sensitivity Analysis

Sibendu Som, Argonne National

Laboratory

60 Million Core-Hours

Computational Design of Novel Multiscale Concrete Rheometers William George, National Institute of Standards and Technology

50 Million Core-Hours

Credible Predictive Simulation Capabilities for Advanced Clean Energy Technology Development Through Uncertainty Quantification Aytekin Gel, ALPEMI

111.5 Million Core-Hours

High-Fidelity Computations of Fuel Assemblies Subjected to Seismic Loads

Elias Balaras, George Washington

University

34 Million Core-Hours 
Large-Eddy Simulation of Turbine Internal Cooling Passages

Gustavo Ledezma, GE Global Research

6 Million Core-Hours

Toward a Longer-Life Core:

Thermal-Hydraulic CFD Simulations

of Deformed Fuel Assemblies

Elia Merzari, Argonne National

Laboratory

72 Million Core-Hours

\section{MATERIALS SCIENCE}

First-Principles Large-Scale Simulations of Interfaces for Energy Conversion and Storage Marco Govoni, The University of Chicago/Argonne National Laboratory 75 Million Core-Hours

Large-Scale Ab Initio Simulation of Crystalline Defects in Mg-Alloys Kaushik Bhattacharya, Caltech 20 Million Core-Hours

Predictive Modeling of Functional Nanoporous Materials

J. Ilja Siepmann, University of Minnesota

120 Million Core-Hours

Revealing the Reversible Electrodeposition Mechanism in Multivalent-Ion Batteries Gerbrand Ceder, Massachusetts Institute of Technology

70 Million Core-Hours

\section{PHYSICS}

Cosmic Frontier Computational End-Station

Salman Habib, Argonne National

Laboratory

115 Million Core-Hours (ALCF: 65M; NERSC: 15M; OLCF: 35M)

An End-Station for Intensity and Energy Frontier Experiments and Calculations

Thomas LeCompte, Argonne National Laboratory

78 Million Core-Hours (ALCF: 62M; NERSC: 16M)
Extreme-Scale Particle Simulation to Carry Out the 2016 FES National Theory and Simulation Performance Target

Choong-Seock Chang, Princeton

Plasma Physics Laboratory

10 Million Core-Hours

Hadronic Light-By-Light Scattering Contribution to the Muon Anomalous Magnetic Moment from Lattice QCD with Chiral Fermions

Thomas Blum, University of

Connecticut

175 Million Core-Hours

Large-Eddy Simulation and Direct Numerical Simulation of Fluid Induced Loads on Reactor Vessel Internals

Milorad Dzodzo, Westinghouse

40 Million Core-Hours

Numerical Simulation of Turbulent Flows in Advanced Steam Generators Aleksandr Obabko, Argonne National Laboratory

25.4 Million Core-Hours

Understanding Helium-Hydrogen Plasma Mediated Tungsten Surface Response to Predict Fusion

Plasma-Facing Component

Performance in ITER

Brian Wirth, University of Tennessee

116 Million Core-Hours (ALCF: 80M;

OLCF: 36M)

Validation Simulations of Macroscopic Burning-Plasma

Dynamics

Jacob King, Tech-X

40 Million Core-Hours

\section{6-2017 ALCC PROJECTS}

\section{BIOLOGICAL SCIENCES}

Molecular Dynamics Studies of Biomass Degradation in Biofuel Production

Emad Tajkhorshid, University of Illinois at Urbana-Champaign

50 Million Core-Hours

\section{CHEMISTRY}

Molecular Modeling of Hot Electron Transfer for Solar Energy Conversion Hanning Chen, George Washington University

16 Million Core-Hours

\section{COMPUTER SCIENCE}

Demonstration of the Scalability of Programming Environments by Simulating Multiscale Applications Robert Voigt, Leidos Inc. 191 Million Core-Hours (ALCF: 151M; OLCF: 4OM)

\section{EARTH SCIENCE}

Delivering the Department of Energy's Next-Generation High-Resolution Earth System Model Peter Thornton, Oak Ridge National Laboratory 211 Million Core-Hours (ALCF: 158M; OLCF: 53M)

\section{ENGINEERING}

Adjoint-Based Optimization via Large-Eddy Simulation of a Fundamental Turbine Stator-Rotor Qiqi Wang, Massachusetts Institute of Technology

15 Million Core-Hours

Computational Study of Cycle-to-Cycle Variation in Dual-Fuel Engines

Ravichandra Jupudi, GE Global

Research

25 Million Core-Hours

Credible Predictive Simulation Capabilities for Advanced Clean Energy Technology Development through Uncertainty Quantification Aytekin Gel, ALPEMI

17 Million Core-Hours

Multiphase Simulations of Nuclear Reactor Flows

Igor Bolotnov, North Carolina State

University

72.1 Million Core-Hours 
Unraveling Silent Owl Flight

to Develop Ultra-Quiet Energy

Conversion Machines

Anupam Sharma, lowa State University

25 Million Core-Hours

\section{MATERIALS SCIENCE}

Computational Engineering of Defects in Soft and Hard Materials for Energy and Quantum Information Applications

Marco Govoni, The University of

Chicago/Argonne National Laboratory

53.7 Million Core-Hours

First-Principles Design and Analysis of Energy-Efficient NanoElectronic

\section{Switches}

Sefa Dag, Globalfoundries

10 Million Core-Hours

First-Principles Design of Magnetic Materials, Models, and Mechanisms Lucas Wagner, University of Illinois at Urbana-Champaign

30 Million Core-Hours

Modeling Helium-Hydrogen Plasma Mediated Tungsten Surface Response to Predict Fusion PlasmaFacing Component Performance in ITER

Brian Wirth, University of Tennessee 95 Million Core-Hours (ALCF: 70M; OLCF: 25M)

Modeling of Intense X-Ray Laser Dynamics in Nanoclusters

Phay Ho, Argonne National Laboratory 10 Million Core-Hours

Predictive Modeling of Functional Nanoporous Materials, Nanoparticle Assembly, and Reactive Systems J. Ilja Siepmann, University of Minnesota

117 Million Core-Hours

The Materials Project: Completing the Space of Elastic and Piezoelectric Tensors

Kristin Persson, Lawrence Berkeley

National Laboratory

36 Million Core-Hours

\section{PHYSICS}

61-Pin Wire-Wrap Turbulent Conjugate-Heat Transfer: V\&V for Industry and SESAME

Elia Merzari, Argonne National

Laboratory

120 Million Core-Hours

Ab Initio Modeling of the Dynamical Stability of HED Plasmas: From

Fusion to Astrophysics

Frederico Fiuza, SLAC National

Accelerator Laboratory

60 Million Core-Hours

An End-Station for Intensity and Energy Frontier Experiments and Calculations

Taylor Childers, Argonne National

Laboratory

106.5 Million Core-Hours

(ALCF: 93.5M; NERSC: 13M)

Exploring Higgs Compositeness Mechanism in the Era of the $14 \mathrm{TeV}$ LHC

George Fleming, Yale University

55 Million Core-Hours

Extreme-Scale Gyrokinetic Particle Simulations to Complete the 2016 OFES National Theory/Simulation Performance Target and to Study the Fundamental Edge Physics Choong-Seock Chang, Princeton Plasma Physics Laboratory 175 Million Core-Hours (ALCF: 100M; OLCF: 75M)

Hadronic Light-by-Light Scattering Contribution to the Muon Anomalous Magnetic Moment from Lattice QCD with Chiral Fermions

Thomas Blum, University of

Connecticut

180 Million Core-Hours

High-Intensity Multibunch Physics in the Fermilab Accelerator Complex James Amundson, Fermilab 50 Million Core-Hours

Muon g-2 Hadronic Vacuum Polarization from Lattice QCD John Laiho, Syracuse University 66 Million Core-Hours
Nuclear Structure for Tests of Fundamental Symmetries and Astroparticle Physics

Calvin Johnson, San Diego State University

30 Million Core-Hours (ALCF: 6M; NERSC: 24M)

Numerical Simulation of Turbulent Flows in Advanced Steam Generators Aleksandr Obabko, Argonne National Laboratory

80 Million Core-Hours

Simulations of Laser Experiments to Study the Origin of Cosmic Magnetic Fields

Petros Tzeferacos, The University of

Chicago

60 Million Core-Hours

\section{DIRECTOR'S DISCRETIONARY PROJECTS}

The following list provides a sampling of the many Director's Discretionary projects at the ALCF.

\section{BIOLOGICAL SCIENCES}

Computing 3D Structures of RNA from Small-Angle X-Ray Scattering Data and Secondary Structures Yuba Bhandari and Yun-Xing Wang, National Cancer Institute 15 Million Core-Hours

\section{CHEMISTY}

First-Principles Monte Carlo Algorithm Development and Implementation in CP2K

Neeraj Rai, Mississippi State University 1.5 Million Core-Hours

First-Principles Simulation of Electronic Excitation Dynamics in Liquid Water and DNA Under Proton Irradiation

Yosuke Kanai, University of North

Carolina

3 Million Core-Hours 
Folding and Stability of an Intrinsically Disordered Domain in Estrogen Receptor

Sichun Yang, Case Western Reserve

University

9.5 Million Core-Hours

Modeling Nonadiabatic

Spin-Forbidden Reaction

Mechanisms in Metal-Sulfur Proteins

Sergey Varganov, University of

Nevada, Reno

1.5 Million Core-Hours

QuanPol QMMM-Style MP2

Simulation Methods

Hui Li, The University of Chicago

1 Million Core-Hours

Scaling of the FMO Method for Heterogeneous Systems

Casper Steinmann Svendsen, University of Bristol

2.4 Million Core-Hours

\section{COMPUTER SCIENCE}

HPC Applications Tuning

Khaled Ibrahim, Lawrence Berkeley

National Laboratory

4 Million Core-Hours

Interfacial Behavior of Alcohol at Water/Organic Biphasic System Baofu Qiao, Argonne National

Laboratory

1.2 Million Core-Hours

Nek Performance Evalutation

Oana Marin, Argonne National

Laboratory

1 Million Core-Hours

Scalable Analysis Methods and In Situ Infrastructure for Extreme-Scale Knowledge Discovery

Venkat Vishwanath, Argonne National Laboratory

2.4 Million Core-Hours

SciDAC Scalable Data Management Analysis and Visualization

Joseph A. Insley and Michael E. Papka,

Argonne National Laboratory

2.5 Million Core-Hours

\section{EARTH SCIENCE}

Contrail-Cirrus Sensitivity to Turbulence Fluctuations Using LES Data

Roberto Paoli, University of Illinois at Chicago

1 Million Core-Hours

\section{ENGINEERING}

Computational Modeling of the

Human Eye

Marco L. Bittencourt, University of

Campinas

5 Million Core-Hours

Direct Numerical Simulation of Bachalo-Johnson Transonic Separated Flow

Philippe Spalart, Boeing

5.9 Million Core-Hours

High-Fidelity Simulations of Complex Turbulent Flows

Krishnan Mahesh, University of

Minnesota

3.9 Million Core-Hours

Influence of Duct Corner Geometry on Secondary Flow: Convergence from Duct to Pipe Flow

Hassan M. Nagib, Illinois Institute of Technology

2.4 Million Core-Hours

Multiphase Simulations of Nuclear Reactor Thermal Hydraulics Igor A. Bolotnov, North Carolina State University

7.6 Million Core-Hours

New Pathways to Stability and Instability in Rayleigh-Taylor

Non-Premixed Flames

Praveen Ramaprabhu, University of North Carolina at Charlotte

1.9 Million Core-Hours

Variable-Density Fluid Dynamics

Paul E. Dimotakis, California Institute of Technology

2.2 Million Core-Hours

\section{MATERIALS SCIENCE}

Ion Transport in Li-S Solid

Ying Li, Argonne National Laboratory 7. 4 Million Core-Hours

Integrating Simulation and Observation: Discovery Engines for Big Data

Rajkumar Kettimuthu and Justin M. Wozniak, Argonne National Laboratory 6 Million Core-Hours

\section{PHYSICS}

Beam Dynamics Simulations for the Advanced Photon Source Upgrade Michael Borland, Argonne National

Laboratory

12.5 Million Core-Hours

NEAMS Neutronics Verification and Validation Simulations

Emily Shemon, Argonne National

Laboratory

5.5 Million Core-Hours

Quantum Monte Carlo for Spin-Orbit Interactions, Spintronic and Van Der Waals Systems

Lubos Mitas, North Carolina State

University

2.3 Million Core-Hours

Particle-In-Cell Scalable Spectral Relativistic

Jean-Luc Vay, Lawrence Berkeley

National Laboratory

1.1 Million Core-Hours

Predicting the Terascale On-Demand with High-Performance Computing Radja Boughezal, Argonne National

Laboratory

9 Million Core-Hours

Using Quantum Monte Carlo for Magnetic Materials

Lucas K. Wagner, University of Illinois at Urbana-Champaign

8 Million Core-Hours

White Dwarf Mergers on Adaptive Meshes

Maximilian Katz, Stony Brook University 2.7 Million Core-Hours 


\begin{abstract}
About Argonne Leadership Computing Facility Argonne's Leadership Computing Facility Division operates the Argonne Leadership Computing Facility as part of the U.S. Department of Energy's effort to provide leadership-class computing resources to the scientific community. The ALCF is supported by the DOE Office of Science, Advanced Scientific Computing Research (ASCR) program.
\end{abstract}

\title{
About Argonne National Laboratory
}

Argonne is a U.S. Department of Energy laboratory managed by UChicago Argonne, LLC, under contract DE-AC02-06CH11357. The Laboratory's main facility is outside of Chicago, at 9700 South Cass Avenue, Argonne, Illinois 60439. For information about Argonne and its pioneering science and technology programs, see www.anl.gov.

\section{Availability of this Report (ANL/ALCF-17/1)}

Online Access: U.S. Department of Energy (DOE) reports produced after 1991 and a growing number of pre-1991 documents are available for free via DOE's SciTech Connect (http://www.osti.gov/scitech/).

Reports not in digital format may be purchased by the public from the National Technical Information Service (NTIS):

U.S. Department of Commerce National Technical Information Service 5301 Shawnee Rd.

Alexandra, VA 22312

www.ntis.gov

phone | 800.553.NTIS (6847) or

703.605 .6000

fax $\mid 703.605 .6900$

orders@ntis.gov

\section{Reports not in digital format are available to DOE and DOE contractors from the Office of Scientific and Technical Information (OSTI):}

U.S. Department of Energy

Office of Scientific and Technical Information P.O. Box 62

Oak Ridge, TN 37831-0062

phone | 865.576.8401

fax | 865.576.5728

reports@adonis.osti.gov

\footnotetext{
Disclaimer

This report was prepared as an account of work sponsored by an agency of the United States Government. Neither the United States Government nor any agency thereof, nor UChicago Argonne, LLC, nor any of their employees or officers, makes any warranty, express or implied, or assumes any legal liability or responsibility for the accuracy, completeness, or usefulness of any information, apparatus, product, or process disclosed, or represents that its use would not infringe privately owned rights. Reference herein to any specific commercial product, process, or service by trade name, trademark, manufacturer, or otherwise, does not necessarily constitute or imply its endorsement, recommendation, or favoring by the United States Government or any agency thereof. The views and opinions of document authors expressed herein do not necessarily state or reflect those of the United States Government or any agency thereof, Argonne National Laboratory, or UChicago Argonne, LLC.
} 



\section{Argonne}

\section{CONTACT}

media@alcf.anl.gov

alcf.anl.gov 